\title{
SEMI-STABLE DEGENERATIONS AND PERIOD SPACES FOR POLARIZED K3 SURFACES
}

\author{
MARTIN C. OLSSON
}

\begin{abstract}
Modular compactifications of moduli spaces for polarized K3 surfaces are constructed using the tools of logarithmic geometry in the sense of Fontaine and Illusie. The relationship between these new moduli spaces and the classical minimal and toroidal compactifications of period spaces are discussed, and it is explained how the techniques of this paper yield models for the latter spaces over number fields. The paper also contains a discussion of Picard functors for log schemes and a logarithmic version of Artin's method for proving representability by an algebraic stack.
\end{abstract}

\section{Contents}

1. Introduction 1

2. Canonical log structures on semi-stable schemes $\quad 7$

3. The log Picard functor: first observations 10

4. The log Picard functor: general definition and representability 14

5. Polarized log K3 surfaces 22

6. The stack of polarized log K3 surfaces 27

7. Log K3 surfaces with level structure 40

8. Minimal and Toroidal compactifications 45

Appendix A. A relative version of M. Artin's method 51

Appendix B. Restatement using log geometry $\quad 57$

$\begin{array}{ll}\text { References } & 60\end{array}$

\section{Introduction}

The purpose of this paper is to introduce certain new moduli spaces obtained as solutions to moduli problems involving "semi-stable K3 surfaces", and to investigate their relationship with minimal and toroidal compactifications of period spaces as well as automorphic forms.

To give a feeling for the types of moduli problems we study, let us give a set theoretic description of the simplest of the stacks considered in this paper. This stack, which depends on the choice of an integer $k>0$, will be denoted by $\mathbb{M}_{2 k}$ and will be defined over $\mathbb{Q}$. The stack $\mathbb{M}_{2 k}$ is quite a large stack, but it will be used to construct various other stacks which perhaps are more interesting (our approach is analogous to the situation for elliptic curves where one constructs various interesting stacks from the moduli stack of generalized elliptic 
curves ([13])). Though the natural description of $\mathbb{M}_{2 k}$ is using logarithmic structures (see below), we can describe the isomorphism classes in $\mathbb{M}_{2 k}(\mathbb{C})$ without using this language. Recall ([20], p. 2, (cf. [31])):

Definition 1.1. A combinatorial K3 surface over an algebraically closed field $\kappa$ is a scheme $X=\bigcup X_{i}$ over $\kappa$ with local normal crossings such that the following hold:

(i) $X$ has one of the following forms:

Type I. $X$ is a smooth K3 surface.

Type II. $X$ is a chain of elliptic ruled surfaces with rational surfaces on either end.

Type III. $X$ is a union of rational surfaces. The double curves on each component form a cycle of rational curves and the dual graph of $X$ is a triangulation of $S^{2}$.

(ii) ( $d$-semistability) There is an isomorphism

$$
\mathcal{E x t}^{1}\left(\Omega_{X}^{1}, \mathcal{O}_{X}\right) \simeq \mathcal{O}_{X_{\text {sing }}} .
$$

(iii) The dualizing sheaf $\omega_{X}$ is trivial.

We say that $X$ is stable if the singular locus is connected (cf. [16], (3.1)). Equivalently, $X$ is stable if $X$ is of type $I$ or $I I I$ or of type $I I$ with no elliptic ruled components.

It is known that the above definition of stability is equivalent to $X$ having no infinitesimal automorphisms ([18], 3.5).

To define what is meant by a polarization on a combinatorial K3 surface $X$, suppose first that we can find a semi-stable degeneration $\tilde{X} \rightarrow \Delta$ over the disc with generic fiber a smooth K3 surface and closed fiber $X$. Then the irreducible components $X_{i}$ of $X$ are divisors on $\tilde{X}$. We let $G \subset \operatorname{Pic}(X)$ be the subgroup generated by the images of the classes of the $X_{i}$. It turns out that $G$ is independent of the choice of the degeneration (see the discussion in (3.2)).

Definition 1.2 (compare with $([16], 3.11)$ ). A polarization on $X$ is a class $\lambda$ in $\operatorname{Pic}(X) / G$ which admits a lifting $\mathcal{L}$ in $\operatorname{Pic}(X)$ such that for some $N>0$ the map $X \rightarrow \bar{X} \subset \mathbb{P}^{r}$ (for some $r$ ) defined by $\mathcal{L}^{N}$ contracts finitely many curves on $X$ to points. The class $\lambda$ is primitive if $\lambda$ is not divisible in $\operatorname{Pic}(X) / G$. The degree of $\lambda$ is defined to be $\mathcal{L}^{2} \in \mathbb{Z}$, for any lifting $\mathcal{L}$ to $\operatorname{Pic}(X)$ (it turns out to be independent of the choices (3.10)).

It is known that for a smooth $\mathrm{K} 3$ surface, the class of a line bundle $\mathcal{L}$ is a polarization in the above sense if and only if $\mathcal{L}$ is numerically effective and $\mathcal{L}^{2}>0$ ([16], 0.6).

Some justification for this definition may be provided by the following observation. If $(X, \mathcal{L})$ is a combinatorial $\mathrm{K} 3$ surface with a line bundle as in the definition, then the class $c(\mathcal{L}) \in H^{2}(X)$ corresponding to $\mathcal{L}$ in the limiting mixed Hodge structure associated to a smoothing of $X$ (when $X$ is endowed with a suitable log structure, $H^{2}(X)$ is the log de Rham cohomology of $X$ ) depends only on $\mathcal{L}$ modulo $G$.

We can now state some of the basic properties of $\mathbb{M}_{2 k}$ :

(1.2.1) $\mathbb{M}_{2 k}$ is a smooth Deligne-Mumford stack over $\mathbb{Q}$ (see (1.4) for our conventions about algebraic stacks).

(1.2.2) $\mathbb{M}_{2 k}$ has a natural open substack $\mathbb{M}_{2 k}^{\mathrm{sm}} \subset \mathbb{M}_{2 k}$ classifying smooth K3 surfaces with primitive polarization of degree $2 k$. The complement $\mathbb{M}_{2 k}-\mathbb{M}_{2 k}^{\mathrm{sm}}$ is a smooth divisor. 
(1.2.3) The isomorphism classes in $\mathbb{M}_{2 k}(\mathbb{C})$ are naturally in bijection with the isomorphism classes of stable $\mathrm{K} 3$ surfaces over $\mathbb{C}$ with primitive polarization of degree $2 k$.

To construct $\mathbb{M}_{2 k}$, we need to make sense of a family of stable, polarized K3 surfaces. This will be done using the tools of logarithmic geometry in the sense of Fontaine and Illusie ([28]). The basic idea is that a combinatorial K3 surface admits a natural structure of a smooth log scheme (in fact $d$-semi-stability is equivalent to the existence of a certain kind of log structure $([27], 11.7(2)))$, and the notion of polarization is naturally defined using the log structure. The stack $\mathbb{M}_{2 k}$ with its log structure coming from the divisor at infinity is then the moduli stack over the category of log schemes classifying polarized log K3 surfaces. In particular, there is a universal polarized $\log \mathrm{K} 3$ surface $(\mathcal{X}, \lambda)$ over $\mathbb{M}_{2 k}$ which induces the bijection $(1.2 .3)$.

It should be noted here that the class of log schemes we consider in this paper is very restrictive, and it would also be interesting to consider more general log smooth morphisms. Our justification for restricting to stable log $K 3$ surfaces is a theorem of Shepherd-Barron ([44], Theorem 2) which implies that any point on the boundary of a toroidal compactification of the period space for polarized Hodge structures coming from $K 3$ surfaces corresponds to the polarized mixed Hodge structure of an element in $\mathbb{M}_{2 k}(\mathbb{C})$. Thus if one is interested in limits of families of smooth $K 3$ surfaces, it is reasonable to restrict attention to stable log $K 3$ surfaces. For example, as we now explain the stacks $\mathbb{M}_{2 k}$ contain enough information about degenerations of polarized $K 3$ surfaces to capture in a purely algebraic manner the minimal and toroidal compactifications of period spaces.

To understand the relationship between $\mathbb{M}_{2 k}$ and period spaces, denote by $\Lambda$ the unique even unimodular lattice of signature $(3,19)([42], 1.1)$, and fix a primitive vector $h \in \Lambda$ with $h^{2}=2 k$. Let $\mathcal{D}_{2 k}$ be the set of filtrations on $\Lambda \otimes \mathbb{C}$ defining a Hodge structure with Hodge numbers those of a K3 surface for which $h$ is of type $(1,1)$. Such a filtration is determined by a line $\mathbb{C} \cdot v \subset \Lambda \otimes \mathbb{C}$ such that $v^{2}=0$ and $v \cdot \bar{v}>0$. Let $\Gamma_{2 k}$ denote the group of isometries of $\Lambda$ fixing the element $h$. The orbifold quotient $\Gamma_{2 k} \backslash \mathcal{D}_{2 k}$, viewed as a stack over the category of smooth complex manifolds, has the following modular interpretation (see for example the proof of $([33], 2.12))$. For any smooth complex manifold $S$, the fiber $\left(\Gamma_{2 k} \backslash \mathcal{D}_{2 k}\right)(S)$ is equivalent to the groupoid of data $(V(\mathbb{Z}), F,<\cdot, \cdot>, \lambda)$ where

(1.2.4) $V(\mathbb{Z})$ is a local system of free $\mathbb{Z}$-modules on $S$.

(1.2.5) $F$ is a holomorphic filtration on $V(\mathbb{Z}) \otimes_{\mathbb{Z}} \mathcal{O}_{S}$.

$$
<\cdot, \cdot>: V(\mathbb{Z}) \times V(\mathbb{Z}) \longrightarrow \mathbb{Z}
$$

is a bilinear symmetric pairing.

(1.2.7) $\lambda$ is a global section of $V(\mathbb{Z})$.

(1.2.8) For each point $s \in S$, there exists an isometry

$$
\beta:\left(V(\mathbb{Z})_{s},<\cdot, \cdot>_{s}\right) \simeq \Lambda
$$

such that $\beta\left(\lambda_{s}\right)=h$ and such that $\beta\left(F_{s}\right)$ defines a point of $\mathcal{D}_{2 k}$ (here $\left(V(\mathbb{Z})_{s}, F_{s},<\cdot, \cdot>_{s}, \lambda_{s}\right)$ denotes the pullback of $(V(\mathbb{Z}), F,<\cdot, \cdot>, \lambda)$ to the point $s)$. 
If $S / \mathbb{C}$ is a smooth variety and $f: X \rightarrow S$ a smooth K3 surface with primitive polarization $\mathcal{L}$ of degree $2 k$, and if $f^{\text {an }}: X^{\text {an }} \rightarrow S^{\text {an }}$ denotes the associated map of analytic spaces, then we obtain a quadruple $\left(R^{2} f_{*}^{\text {an }} \mathbb{Z}, F,<\cdot,>, c\left(\mathcal{L}^{\text {an }}\right)\right)$ as above on $S^{\text {an }}$ by letting $F$ be the Hodge filtration on

$$
\left(R^{2} f_{*}^{\text {an }} \mathbb{Z}\right) \otimes \mathcal{O}_{S^{\text {an }}} \simeq R^{2} f_{*}^{\text {an }} \Omega_{X^{\text {an }} / S^{\text {an }}},
$$

$<\cdot, \cdot>$ the pairing defined by cup-product, and $c\left(\mathcal{L}^{\text {an }}\right)$ the first Chern class of $\mathcal{L}^{\text {an }}$. Hence we obtain an analytic map

$$
p_{S^{\mathrm{an}}}: S^{\mathrm{an}} \longrightarrow \Gamma_{2 k} \backslash \mathcal{D}_{2 k}
$$

Now the orbifold $\Gamma_{2 k} \backslash \mathcal{D}_{2 k}$ has a natural structure of an algebraic stack over $\mathbb{C}$ by $([9], 10.11)$, and by ([10], 3.10) the map (1.2.10) is induced by a unique algebraic map $p_{S}: S \rightarrow \Gamma_{2 k} \backslash \mathcal{D}_{2 k}$. If $\left(X^{\prime}, \mathcal{L}^{\prime}\right)$ is a second primitively polarized smooth K3 surface over $S$ and if $p_{S}^{\prime}: S \rightarrow$ $\Gamma_{2 k} \backslash \mathcal{D}_{2 k}$ denotes the associated morphism, then an isomorphism $(X, \mathcal{L}) \simeq\left(X^{\prime}, \mathcal{L}^{\prime}\right)$ induces an isomorphism of functors $p_{S} \simeq p_{S}^{\prime}$, and hence we obtain a morphism of algebraic stacks

$$
p: \mathbb{M}_{2 k}^{\mathrm{sm}} \otimes \mathbb{C} \longrightarrow \Gamma_{2 k} \backslash \mathcal{D}_{2 k}
$$

which we refer to as the period map. The global Torelli theorem ([16], 1.2) implies that (1.2.11) is representable, étale, generically an isomorphism, and a bijection on geometric points. More generally, if $\Gamma \subset \Gamma_{2 k}$ is an arithmetic subgroup, the quotient $\Gamma \backslash \mathcal{D}_{2 k}$ has a natural structure of an algebraic stack and receives a representable, étale morphism from a stack classifying polarized K3 surfaces with suitable level structure.

It is not quite true, however, that $\mathbb{M}_{2 k}^{\mathrm{sm}} \otimes \mathbb{C}$ is isomorphic to $\Gamma_{2 k} \backslash \mathcal{D}_{2 k}$. As mentioned above, the map (1.2.11) is étale and the two stacks have the same $\mathbb{C}$-valued points. However, the objects of $\Gamma_{2 k} \backslash \mathcal{D}_{2 k}$ have more automorphisms than the objects of $\mathbb{M}_{2 k}^{\mathrm{sm}}$ (because of so-called -2 -curves; see ([16] 1.2)). The philosophy of this paper is that for many questions the difference between the two stacks can be neglected.

This is at least true with regards to defining automorphic forms. If $\omega$ denotes the universal line bundle on $\Gamma_{2 k} \backslash \mathcal{D}_{2 k}$, then the pullback $p^{*} \omega$ is isomorphic to $\left(f_{*} \Omega_{\mathcal{X}^{*} / \mathbb{M}_{2 k}^{\text {sm }}}^{2}\right) \otimes \mathbb{C}$ where $f$ : $\mathcal{X}^{*} \rightarrow \mathbb{M}_{2 k}^{\text {sm }}$ is the universal K3 surface. We show (8.3) that for every integer $l$, the natural map

$$
H^{0}\left(\Gamma_{2 k} \backslash \mathcal{D}_{2 k}, \omega^{\otimes l}\right) \longrightarrow H^{0}\left(\mathbb{M}_{2 k}^{\mathrm{sm}},\left(f_{*} \Omega_{\mathcal{X}^{*} / \mathbb{M}_{2 k}^{\mathrm{sm}}}^{2}\right)^{\otimes l}\right) \otimes \mathbb{C}
$$

is an isomorphism. Moreover, if $\mathcal{X} \rightarrow \mathbb{M}_{2 k}$ is the universal log K3 surface, then the natural map

$$
H^{0}\left(\mathbb{M}_{2 k},\left(f_{*} \Omega_{\mathcal{X} / \mathbb{M}_{2 k}}^{2}\right)^{\otimes l}\right) \longrightarrow H^{0}\left(\mathbb{M}_{2 k}^{\mathrm{sm}},\left(f_{*} \Omega_{\mathcal{X}^{*} / \mathbb{M}_{2 k}^{\mathrm{sm}}}^{2}\right)^{\otimes l}\right)
$$

is an isomorphism as well (8.4). This result enables one for example to think of a cusp form of weight $l$ with respect to $\Gamma_{2 k}$ as a "rule" which to any polarized $\log$ K3 surface $f: X \rightarrow T$ over some log scheme $T$ associates a section of $\left(f_{*} \Omega_{X / T}^{2}\right)^{\otimes l}$ (where $\Omega_{X / T}^{2}$ denotes the second wedge product of the log differentials) which vanishes at the points $t \in T$ over which the fiber is singular (in the usual sense).

Once we have this interpretation of automorphic forms as sections over the moduli stack of $\log$ K3 surfaces, we can also construct minimal and toroidal compactifications of the period spaces $(([9])$ and $([8]))$. To explain this, let us consider the situation of a neat arithmetic subgroup $\Gamma \subset \Gamma_{2 k}$ so that $\Gamma \backslash \mathcal{D}_{2 k}$ is a scheme and not only an algebraic stack. We will 
construct a Deligne-Mumford stack $\mathbb{M}_{2 k, \Gamma}$, which will be defined over a certain number field $F_{\Gamma} \subset \mathbb{C}$, classifying polarized log K3 surfaces with " $\Gamma$-level structure" (7.10-7.13). Over $\mathbb{M}_{2 k, \Gamma}$ there is a universal $\log \mathrm{K} 3$ surface $f: \mathcal{X} \rightarrow \mathbb{M}_{2 k, \Gamma}$ and if $\Omega_{\mathcal{X} / \mathbb{M}_{2 k, \Gamma}^{2}}$ denotes the second wedge product of the log differentials, then we show that $\left(f_{*} \Omega_{\mathcal{X} / \mathbb{M}_{2 k, \Gamma}}^{2}\right)^{\otimes l}$ is generated by global sections for suitable $l$ and that $\Gamma\left(\mathbb{M}_{2 k, \Gamma},\left(f_{*} \Omega_{\mathcal{X} / \mathbb{M}_{2 k, \Gamma}}^{2}\right){ }^{\otimes l}\right)$ is finitely generated. The image of the map to projective space defined by $f_{*} \Omega_{\mathcal{X} / \mathbb{M}_{2 k, \Gamma}}^{2}$ is then a model $\left(\Gamma \backslash \mathcal{D}_{2 k}\right)_{F_{\Gamma}}^{*}$ for the minimal compactification of $\Gamma \backslash \mathcal{D}_{2 k}$ over $F_{\Gamma}$. In particular, the image is a normal projective scheme.

Once we have the minimal compactification, we can construct certain toroidal compactifications by blowing up sheaves of ideals on $\left(\Gamma \backslash \mathcal{D}_{2 k}\right)_{\mathbb{C}}^{*}$ and normalizing (see the discussion in (8.6)). However, we will show that the relevant sheaves of ideals are already defined over $F_{\Gamma}$, and hence we obtain models for toroidal compactifications over $F_{\Gamma}$. In fact we show more. Let $\Sigma$ denote the data needed to define a projective toroidal compactification of $\Gamma \backslash \mathcal{D}_{2 k}$ (a " $\Gamma$-admissible collection of fans"), and let $\left(\overline{\Gamma \backslash \mathcal{D}_{2 k}}\right)$ be the associated compactification. We associate to $\Sigma$ a quasi-separated algebraic space $\mathbb{M}_{2 k, \Gamma, \Sigma}$ over $F_{\Gamma}$ with the following properties:

(1.2.12) There is a polarized log $\mathrm{K} 3$ surface over $\mathbb{M}_{2 k, \Gamma, \Sigma}$ which is the universal solution to a certain moduli problem.

(1.2.13) There is an étale quasi-finite map $p_{F_{\Gamma}}: \mathbb{M}_{2 k, \Gamma, \Sigma} \rightarrow\left(\overline{\Gamma \backslash \mathcal{D}_{2 k}}\right)_{F_{\Gamma}}$ to a proper scheme $\left(\overline{\Gamma \backslash \mathcal{D}_{2 k}}\right)_{F_{\Gamma}}$ which comes equipped with a canonical isomorphism $\left(\overline{\Gamma \backslash \mathcal{D}_{2 k}}\right)_{F_{\Gamma}} \otimes \mathbb{C} \simeq\left(\overline{\Gamma \backslash \mathcal{D}_{2 k}}\right)$.

(1.2.14) If $V \subset\left(\overline{\Gamma \backslash \mathcal{D}_{2 k}}\right)_{F_{\Gamma}}$ denotes the image of $p_{F_{\Gamma}}$, then $\left(\overline{\Gamma \backslash \mathcal{D}_{2 k}}\right)_{F_{\Gamma}}-V$ has codimension $\geq 2$, and the map $p: \mathbb{M}_{2 k, \Gamma, \Sigma} \rightarrow V$ is universal for maps from $\mathbb{M}_{2 k, \Gamma, \Sigma}$ to separated algebraic spaces.

Moreover, the relationship between $\mathbb{M}_{2 k, \Gamma, \Sigma}$ and $V$ is well-understood in terms of elementary modifications ([19], 12-14) just as in the case of $\mathbb{M}_{2 k}^{\mathrm{sm}} \otimes \mathbb{C}$ and $\Gamma_{2 k} \backslash \mathcal{D}_{2 k}$.

The paper is organized as follows.

In section 2 we review some results from the paper ([40]) which will play an important role in what follows.

In sections 3 and 4 we define the log Picard functor of a morphism of log algebraic spaces and study its basic properties. In section 3 we study the geometric points of this functor which have a natural structure of a group. Moreover, in the case of a surface we show that there is a natural intersection pairing on this group (3.9). This will enable us to speak of the degree of a polarization in what follows. In section 4 we consider the case of an arbitrary base. We define the log Picard functor for an arbitrary morphism and prove that it is representable by an algebraic space (4.6) when the morphism satisfies a logarithmic analogue of cohomological flatness in dimension 0. This is a logarithmic analogue of a theorem of M. Artin ([5], 7.3). We have also included a brief discussion in section 3 of the logarithmic Picard group for log curves which may be of some independent interest. The log Picard group for log curves has previously been studied by T. Kajiwara ([26]).

We introduce the notion of a polarized log K3 surface in section 5, and discuss some basic properties. Then in section 6 we define the stack $\mathbb{M}_{2 k}$ and prove that it is a Deligne-Mumford stack using the results of appendix B. 
Once we have $\mathbb{M}_{2 k}$ we can define various other algebraic stacks classifying log K3 surfaces with level structure. This we do in section 7 .

Finally in section 8 we discuss the minimal and toroidal compactifications of $\Gamma \backslash \mathcal{D}_{2 k}$. We also show that the Hodge bundle with logarithmic connection and Hodge filtration on $\mathbb{M}_{2 k, \Gamma, \Sigma}$ obtained from the universal family descends to the scheme $\left(\overline{\Gamma \backslash \mathcal{D}_{2 k}}\right)_{F_{\Gamma}}$ (notation as above). The results of this section are perhaps more naturally viewed in the context of Shimura varieties (i.e. replace $\Gamma \backslash \mathcal{D}_{2 k}$ by an appropriate Shimura variety), but in order not to introduce too much machinery into the paper we have chosen not to discuss this. We hope that it is clear how to proceed for the reader familiar with this subject.

We have included two appendices, the results of which are used in the proof that $\mathbb{M}_{2 k}$ is algebraic. The first appendix discusses a version of M. Artin's theorem ([2], 5.3) with an algebraic stack as a base. The main result is (A.12). In the second appendix we reformulate (A.12) in the case when the base is $\mathcal{L} o g$ (see (1.4), and we obtain a logarithmic version of $([2], 5.3)$.

1.3. Acknowledgements. We would like to acknowledge the strong influence on this paper of the work of R. Friedman ([16]) and R. Friedman and F. Scattone ([20]) on partial compactifications of period spaces for K3 surfaces. In addition, we found N.I. Shepherd-Barron's paper $([44])$ very helpful.

Much of this work was done at the Mathematical Sciences Research Institute in Berkeley, CA. The author would like to thank this institution for its excellent working conditions. Thanks are also due to A. Abbes, L. Illusie, and A. Ogus for their encouragement which led the author to return to the subject of K3 surfaces. Special thanks are due to J. Starr for numerous enlightening conversations. We also want to thank the referee for helpful comments. The author was partially supported by an NSF post-doctoral research fellowship.

1.4. Conventions and Prerequisites. We assume that the reader is familiar with logarithmic geometry at the level of the paper $([28])$ as well as the stack-theoretic approach introduced in ([39]). We freely use the notation of these two papers. In particular, if $S$ is a fine $\log$ scheme, we denote by $\mathcal{L}_{o g_{S}}$ the algebraic stack whose objects are morphisms of fine $\log$ schemes $X \rightarrow S$, and whose morphisms are given by strict morphisms over $S$. We sometimes write $\mathcal{L} o g$ for $\mathcal{L}_{\left.o g_{(\operatorname{Spec}}(\mathbb{Z}), \mathcal{O}_{\text {Spec } \mathbb{Z})}^{*}\right)}$. Only fine log schemes are considered in this paper, so we often omit the adjective "fine".

Regarding algebraic stacks we follow the conventions of ([32]), except we do not assume that our stacks are quasi-separated. More precisely, by an algebraic stack we mean a stack $X$ in the sense of ([32], 3.1) satisfying the following:

(1.4.1) The diagonal

$$
\Delta: X \longrightarrow x \times x
$$

is representable and locally of finite type;

(1.4.2) There exists a surjective smooth morphism $X \rightarrow X$ from a scheme.

We call an algebraic stack $\mathcal{X}$ for which there exists an étale surjective cover $X \rightarrow X$ a Deligne-Mumford stack.

The reader is assumed to be familiar with algebraic stacks. 
In sections 5-8 all algebraic spaces are defined over $\mathbb{Q}$ unless we state otherwise.

\section{Canonical log structures on semi-stable schemes}

In this section we review some definitions and results from $([40])$ which will be used in what follows.

2.1. If $R$ is a complete discrete valuation ring with uniformizer $\pi$, and $X / R$ is a regular algebraic space with smooth generic fiber and closed fiber $X_{0}$ a divisor with normal crossings, then the morphism $X \rightarrow \operatorname{Spec}(R)$ can naturally be viewed as the underlying morphism of algebraic spaces of a log smooth, integral, and vertical morphism of log algebraic spaces by endowing $\operatorname{Spec}(R)$ and $X$ with the pushforwards of the trivial log structures on the generic fibers $([28], 3.7(2))$. The purpose of the paper $([40])$ was to investigate to what extent the log structures on $X$ and $\operatorname{Spec}(R)$ are intrinsic invariants of the above situation, and to generalize to other bases.

Definition 2.2. A morphism $f: X \rightarrow S$ is semi-stable if it is flat and if for every geometric point $\bar{s} \rightarrow S$ the fiber $X_{\bar{s}}$ is étale locally isomorphic to

$$
\kappa(\bar{s})\left[x_{1}, \ldots, x_{n}\right] /\left(x_{1} \cdots x_{r}\right)
$$

for some $n$ and $r$.

We will often consider the following class of log morphisms in what follows:

Definition 2.3. A morphism $\left(f, f^{b}\right):\left(X, \mathcal{M}_{X}\right) \rightarrow\left(S, \mathcal{M}_{S}\right)$ from a log algebraic space to a $\log$ scheme is $\log$ semi-stable if $f$ is $\log$ smooth, integral, and vertical and if the underlying morphism $f: X \rightarrow S$ is semi-stable (recall that a morphism of fine log schemes $g:\left(U, \mathcal{M}_{U}\right) \rightarrow$ $\left(V, \mathcal{M}_{V}\right)$ is called vertical if the cokernel in the category of sheaves of integral monoids of the map $g^{*} \mathcal{M}_{V} \rightarrow \mathcal{M}_{U}$ is a sheaf of groups).

2.4. Fix a proper semi-stable morphism $f: X \rightarrow S$ from an algebraic space to a scheme $S$ of finite type over an excellent Dedekind ring, and consider the functor

$$
\operatorname{IVLS}_{X / S}:(\log \text { schemes over } S) \longrightarrow(\text { Set })
$$

which to any $\log$ scheme $\left(T, \mathcal{M}_{T}\right)$ associates the isomorphism classes of pairs $\left(\mathcal{M}_{X_{T}}, f^{b}\right)$, where $\mathcal{M}_{X_{T}}$ is a $\log$ structure on $X_{T}:=X \times_{S} T$ and $f^{b}: \operatorname{pr}_{2}^{*} \mathcal{M}_{T} \rightarrow \mathcal{M}_{X_{T}}$ is a morphism of $\log$ structures such that

$$
\left(X_{T}, \mathcal{M}_{X_{T}}\right) \longrightarrow\left(T, \mathcal{M}_{T}\right)
$$

is $\log$ semi-stable. The following theorem is proven in $([40], 1.2)$ (the result is stated there only for the case when $X$ is a scheme, but the argument generalizes verbatim to the case of an algebraic space):

Theorem 2.5. IVLS $_{X / S}$ is representable by a log scheme over $S$. The tautological map from the underlying scheme of $\operatorname{IVLS}_{X / S}$ to $S$ is a monomorphism of finite type.

Remark 2.6. If $S=\operatorname{Spec}(\kappa)$ for an algebraically closed field $\kappa$, then $\operatorname{IVLS}_{X / S}$ is non-empty if and only if $X$ is $d$-semi-stable in the sense of ([17], 1.13). This follows from ([40], 3.14) or $([27], 11.7(2))$.

The theorem prompts the following definition: 
Definition 2.7. A proper log semi-stable morphism $\left(f, f^{b}\right):\left(X, \mathcal{M}_{X}\right) \longrightarrow\left(S, \mathcal{M}_{S}\right)$ is special if the tautological map $\left(S, \mathcal{M}_{S}\right) \rightarrow \operatorname{IVLS}_{X / S}$ is strict (recall that a morphism of log schemes $f: Y \rightarrow Z$ is strict if the map $f^{*} \mathcal{M}_{Z} \rightarrow \mathcal{M}_{Y}$ is an isomorphism).

2.8. It follows from $(2.5)$ that if $\left(f, f^{b}\right):\left(X, \mathcal{M}_{X}\right) \rightarrow\left(S, \mathcal{M}_{S}\right)$ is a proper log semi-stable morphism, then there exists a cartesian diagram in the category of log algebraic spaces

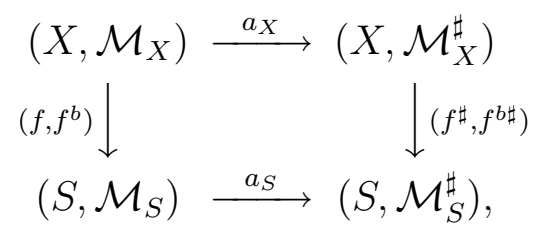

where the morphisms $\stackrel{\circ}{a}_{S}$ and $\stackrel{\circ}{a}_{X}$ are the identity morphisms and $\left(f^{\sharp}, f^{b \sharp}\right)$ is special. In fact, it is shown in ([40], 2.6) that the additional data of the diagram (2.8.1) is unique up to unique isomorphism (and not just unique up to isomorphism). In other words, given a second cartesian diagram

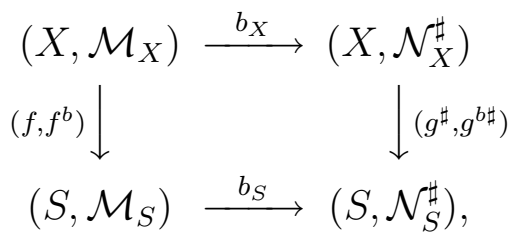

where $\stackrel{\circ}{b}_{X}$ and $\stackrel{\circ}{b}_{S}$ are the identity maps and $\left(g^{\sharp}, g^{b \sharp}\right)$ is special, there exists a unique isomorphisms

$$
i_{1}:\left(X, \mathcal{N}_{X}^{\sharp}\right) \longrightarrow\left(X, \mathcal{M}_{X}^{\sharp}\right), \quad i_{2}:\left(S, \mathcal{N}_{S}^{\sharp}\right) \longrightarrow\left(S, \mathcal{M}_{S}^{\sharp}\right)
$$

such that $i_{2} \circ\left(g^{\sharp}, g^{b \sharp}\right)=\left(f^{\sharp}, f^{b \sharp}\right) \circ i_{1}, i_{1} \circ b_{X}=a_{X}$, and $i_{2} \circ b_{S}=a_{S}$. This has the following corollary:

Corollary 2.9. Let $\left(X, \mathcal{M}_{X}\right) /\left(S, \mathcal{M}_{S}\right)$ be a proper and special log semi-stable morphism. Then there are no non-trivial automorphisms of $\left(X, \mathcal{M}_{X}\right)$ over $\left(S, \mathcal{M}_{S}\right)$ which are the identity on $X$.

2.10. If $\left(f, f^{b}\right):\left(X, \mathcal{M}_{X}\right) \rightarrow\left(S, \mathcal{M}_{S}\right)$ is a proper log semi-stable morphism and if $\left(g, g^{b}\right)$ : $\left(S, \mathcal{M}_{S}\right) \rightarrow \operatorname{IVLS}_{X / S}$ denotes the tautological morphism of log schemes, then by $([28],(1.4 .1))$ the morphism $\left(f, f^{b}\right)$ is special if and only if the induced map $\bar{g}^{b}: g^{-1} \overline{\mathcal{M}}_{\mathrm{IVLS}_{X / S}} \rightarrow \overline{\mathcal{M}}_{S}$ is an isomorphism. Therefore, $\left(f, f^{b}\right)$ is special if and only if for every geometric point $\bar{s} \rightarrow S$, the fiber $\left(X_{\bar{s}}, \mathcal{M}_{X_{\bar{s}}}\right) \rightarrow\left(\bar{s}, \mathcal{M}_{\bar{s}}\right)$ is special (here $\mathcal{M}_{\bar{s}}$ denotes the pullback of $\mathcal{M}_{S}$ to the point $\bar{s}$ ).

Now in the case when $S$ is the spectrum of a separably closed field $\kappa$, we can give an alternate description of what it means for $\left(f, f^{b}\right)$ to be special. First of all, it follows from the proof of (2.5) that $\overline{\mathcal{M}}_{S}$ is a free monoid of finite rank and that for every geometric point $\bar{x} \rightarrow X$ the monoid $\overline{\mathcal{M}}_{X, \bar{x}}$ is also free of finite rank. Moreover, for suitable isomorphisms $\overline{\mathcal{M}}_{S} \simeq \mathbb{N}^{r}$ and $\overline{\mathcal{M}}_{X, \bar{x}} \simeq \mathbb{N}^{r+s}$ the natural map

$$
f^{b}: \overline{\mathcal{M}}_{S} \longrightarrow \overline{\mathcal{M}}_{X, \bar{x}}
$$

is of the form

$$
e_{i} \mapsto\left\{\begin{array}{cl}
e_{i} & \text { if } i \neq r \\
e_{r}+e_{r+1}+\cdots+e_{r+s} & \text { if } i=r .
\end{array}\right.
$$


In particular, if $\bar{x}$ is a singular point of $X$, then there exists a unique element among the irreducible elements of $\overline{\mathcal{M}}_{S}$ whose image in $\overline{\mathcal{M}}_{X, \bar{x}}$ is not irreducible. Thus we obtain a map

$s: X_{\text {sing }} \longrightarrow\left\{\right.$ irreducible elements of $\left.\overline{\mathcal{M}}_{S}\right\}$.

The condition that $\left(f, f^{b}\right)$ is special is then equivalent to the statement that the map $s$ induces a bijection between the set of connected components of the singular locus of $X$ and the set of irreducible elements in $\overline{\mathcal{M}}_{S}$. In other words, if $\left(f, f^{b}\right)$ is special, then $s$ induces a canonical isomorphism $\overline{\mathcal{M}}_{S} \simeq \mathbb{N}^{\mathcal{C}(X)}$, where $\mathcal{C}(X)$ is defined as follows:

Notation 2.11. If $X$ is a scheme over a separably closed field, then we denote by $\mathcal{C}(X)$ the connected components of the singular locus of $X$.

2.12. Suppose now that $S$ is the spectrum of a complete local ring $\hat{A}$ with separably closed residue field and that $\left(f, f^{b}\right):\left(X, \mathcal{M}_{X}\right) \rightarrow\left(S, \mathcal{M}_{S}\right)$ is special. Then by the preceding paragraph, we have a canonical isomorphism $H^{0}\left(S, \overline{\mathcal{M}}_{S}\right) \simeq \mathbb{N}^{\mathcal{C}\left(X_{0}\right)}$, where $X_{0}$ denotes the closed fiber of $X$. For each $c \in \mathcal{C}\left(X_{0}\right)$, let $\overline{\mathcal{M}}_{c} \subset \overline{\mathcal{M}}_{X}$ be the subsheaf of sections $m \in \overline{\mathcal{M}}_{X}$ such that étale locally there exists $n \in \overline{\mathcal{M}}_{X}$ such that $m+n$ is a multiple of the image of the $c$-th standard generator of $\mathbb{N}^{\mathcal{C}\left(X_{0}\right)} \simeq \overline{\mathcal{M}}_{S}$. Denote by $\mathcal{M}_{c} \subset \mathcal{M}_{X}$ the inverse image of $\overline{\mathcal{M}}_{c}$. It follows from a local calculation that there is a canonical decomposition in the category of log structures on $X$ (here the sum denotes pushout over $\mathcal{O}_{X}^{*}$ )

$$
\mathcal{M}_{X} \simeq \bigoplus_{c \in \mathcal{C}\left(X_{0}\right), \mathcal{O}_{X}^{*}} \mathcal{M}_{c}
$$

and that the map $f^{-1} \overline{\mathcal{M}}_{S} \rightarrow \overline{\mathcal{M}}_{X}$ is compatible with the $\mathcal{C}\left(X_{0}\right)$-grading.

Example 2.13. If $z \in X$ is a point of the $c$-th component of the singular locus, then in an étale neighborhood of $z$ we can find a chart

$$
X \longrightarrow \operatorname{Spec}\left(\hat{A}\left[x_{1}, \ldots, x_{n}\right] /\left(x_{1} \cdots x_{r}-t\right)\right), \quad t \in \hat{A}
$$

sending $z$ to the point $\left\{x_{i}=0\right\}_{i=1}^{n}$. In this neighborhood $\mathcal{M}_{c}$ is simply the log structure associated to the map $\mathbb{N}^{r} \rightarrow \mathcal{O}_{X}$ sending $e_{i}$ to $x_{i}$.

2.14. It will also be useful later to observe that the set of points $Z$ of $X$ where $f$ is not smooth has a natural structure of an algebraic space. Let $Z_{c}$ be the $c$-th connected component of $Z$ (note that by $([4], 3.1)$ the connected components of $Z$ are again indexed by $\mathcal{C}\left(X_{0}\right)$ ). Then the sheaf

$$
\mathcal{E} x t^{1}\left(\Omega_{X / \hat{A}}^{1}, \mathcal{O}_{X}\right)
$$

is supported on $Z$, and we let $J_{c}$ be the ideal sheaf which in a neighborhood of $z \in Z_{c}$ is equal to the annihilator of (2.14.1) and trivial elsewhere. It follows from ([40], 3.14 (1)), that if we choose an étale neighborhood $U$ of $z$ and an étale morphism giving a chart to

$$
\operatorname{Spec}\left(\hat{A}\left[x_{1}, \ldots, x_{n}\right] /\left(x_{1} \cdots x_{r}-t_{c}\right)\right)
$$

for some $n, r$, and $t_{c} \in \mathfrak{m}_{A}$, then $J_{c}$ is the ideal

$$
\left(x_{1} \cdots \widehat{x}_{j} \cdots x_{r}\right)_{j=1}^{r},
$$

and the closed subspace of $X$ defined by $J_{c}$ is set theoretically equal to $Z_{c}$. 
In the case when $t_{c}=0$, this also gives a description of $\overline{\mathcal{M}}_{c}$. Let $\nu_{c}: \tilde{X}_{c} \rightarrow X$ be the blow-up of the ideal $J_{c}$.

Proposition 2.15. Assume that $t_{c}=0$. Then there exists a unique isomorphism $\sigma: \overline{\mathcal{M}}_{c} \simeq$ $\nu_{c *} \mathbb{N}$ with the property that for every geometric point $\bar{x} \rightarrow X$ with inverse images $\bar{x}_{1}, \ldots, \bar{x}_{r} \in$ $\tilde{X}_{c}$, the induced map

$$
\overline{\mathcal{M}}_{c, \bar{x}} \longrightarrow\left(\nu_{c *} \mathbb{N}\right)_{\bar{x}} \simeq \oplus_{\bar{x}_{i}} \mathbb{N}_{\bar{x}_{i}}
$$

sends a primitive element $e \in \overline{\mathcal{M}}_{c, \bar{x}}$ with lifting $\tilde{e} \in \mathcal{M}_{c, \bar{x}}$ to the generator of $\mathbb{N}_{\bar{x}_{i}}$ for the unique point $\bar{x}_{i}$ for which $\tilde{e}$ maps to zero in $\mathcal{O}_{\tilde{X}_{i}, \bar{x}_{i}}$.

Proof. It suffices to prove the proposition after replacing $X$ by an étale cover so we may assume that

$$
X=\operatorname{Spec}\left(\hat{A}\left[x_{1}, \ldots, x_{n}\right] /\left(x_{1} \cdots x_{r}\right)\right)
$$

with the natural $\log$ structure, and that $Z_{c}$ is equal to the singular locus. Let $X_{j} \subset X$ be the closed subscheme defined by $x_{j}=0$. In this case, $\tilde{X}_{i}$ is equal to

$$
\coprod_{j=1}^{r} X_{j}
$$

Define a map $\nu_{c}^{-1} \mathbb{N}^{r} \rightarrow \mathbb{N}$ by sending the $j$-th generator $e_{j}$ to 1 on $X_{j}$ and all the rest of the generators to zero. The resulting map $\mathbb{N}^{r} \rightarrow \nu_{c *} \mathbb{N}$ then induces the required isomorphism $\sigma$ on $X$ which is easily seen to be unique and hence defined globally.

\section{The log Picard functor: first observations}

3.1. Let

$$
\left(f, f^{b}\right):\left(X, \mathcal{M}_{X}\right) \rightarrow\left(S, \mathcal{M}_{S}\right)
$$

be a special log semi-stable morphism, with $S$ the spectrum of a separably closed field $\kappa$. In this section we make some basic observations about the group $H^{1}\left(X, \mathcal{M}_{X}^{\text {gp }}\right)$. This group had previously been suggested as a good candidate for a logarithmic Picard group ([25], $3.3)$, and is naturally identified with the $\kappa$-valued points of the logarithmic Picard functor $\operatorname{Pic}\left(\left(X, \mathcal{M}_{X}\right) /\left(S, \mathcal{M}_{S}\right)\right)$ defined in the next section (4.5). The discussion of curves (3.4)-(3.8) is not used anywhere else in the paper and may be omitted. For a more thorough discussion of the log Picard group for curves see Kajiwara's paper ([26]).

Observation 3.2. For each $c \in \mathcal{C}(X)$, let $\nu_{c}: \tilde{X}_{c} \rightarrow X$ be the blow-up of the $c$-th connected component of the singular locus of $X$ as in (2.15), so that we have an exact sequence

$$
0 \longrightarrow \mathcal{O}_{X}^{*} \longrightarrow \mathcal{M}_{X}^{\mathrm{gp}} \longrightarrow \oplus_{c} v_{c *} \mathbb{Z} \longrightarrow 0 .
$$

Since $H^{1}\left(\tilde{X}_{c}, \mathbb{Z}\right)=0\left([7]\right.$, IX.3.6), the sequence $(3.2 .1)$ identifies $H^{1}\left(X, \mathcal{M}_{X}^{\text {gp }}\right)$ with the quotient of the usual Picard group $\operatorname{Pic}(X)$ by a map

$$
\oplus_{c} H^{0}\left(\tilde{X}_{c}, \mathbb{Z}\right) \longrightarrow \operatorname{Pic}(X)
$$

Choose an isomorphism $\mathcal{M}_{\kappa} \simeq \mathbb{N}^{r} \oplus \kappa^{*}$ (for some $r$ ), and let

$$
\left(X, \mathcal{M}_{X}^{\prime}\right) \longrightarrow\left(\operatorname{Spec}(\kappa), \mathbb{N} \oplus \kappa^{*}\right)
$$


be the log algebraic space obtained from $\left(X, \mathcal{M}_{X}\right)$ by base change via the map

$$
\left(\operatorname{Spec}(\kappa), \mathbb{N} \oplus \kappa^{*}\right) \rightarrow\left(\operatorname{Spec}(\kappa), \mathbb{N}^{r} \oplus \kappa^{*}\right), \quad \operatorname{sum}: \mathbb{N}^{r} \rightarrow \mathbb{N}
$$

If $\nu: \tilde{X} \rightarrow X$ denotes the normalization of $X$, then by the same reasoning as in the proof of (2.15) there is a natural isomorphism $\overline{\mathcal{M}}_{X}^{\prime} \simeq \nu_{*} \mathbb{N}$. For each $c \in \mathcal{C}(X)$ there is a natural map $\tilde{X} \rightarrow \tilde{X}_{c}$, and hence natural maps $s_{c}: \nu_{c *} \mathbb{N} \rightarrow \nu_{*} \mathbb{N}$. Moreover, from the commutativity of the diagram

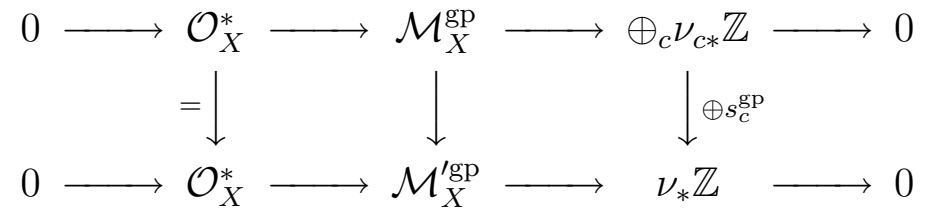

we conclude that $H^{1}\left(X, \mathcal{M}_{X}^{\mathrm{gp}}\right)$ is the cokernel of a map

$$
(\mathbb{Z})^{\{\text {connected components of } \tilde{X}\}} \longrightarrow H^{1}\left(X, \mathcal{O}_{X}^{*}\right) .
$$

This map has the following interpretation. Suppose $X$ has a smoothing $\mathcal{X} / R$ over some discrete valuation ring $R$ with residue field $\kappa$. Then the different irreducible components $X_{i}$ of $X$ are divisors in $\mathcal{X}$ and the image of the generator corresponding to $X_{i}$ in (3.2.3) is equal to the image of the class of the divisor $X_{i}$.

Observation 3.3. Continuing the discussion in the previous observation, let $\mathcal{L}_{X_{i}} \in H^{1}\left(X, \mathcal{O}_{X}^{*}\right)$ be the image of the class corresponding to $X_{i}$ under (3.2.3). Even without the existence of a smoothing, we can show in the following way that $\left.\mathcal{L}_{X_{i}}\right|_{X_{j}}(j \neq i)$ is isomorphic to $\mathcal{O}_{X_{j}}\left(-D_{i j}\right)$, where $D_{i j}$ denotes the intersection $X_{i} \cap X_{j}$. Let $e_{i} \in \overline{\mathcal{M}}_{X}^{\text {gp }}$ be the section corresponding to $X_{i}$ so that $\mathcal{L}_{X_{i}}$ is the $\mathcal{O}_{X}^{*}$-torsor of liftings of $e_{i} \in \overline{\mathcal{M}}_{X}^{\mathrm{gp}}$ to $\mathcal{M}_{X}^{\mathrm{gp}}$. Let $U \rightarrow X$ be an étale morphism, and suppose we have a lifting $\tilde{e}_{i}$ of $e_{i}$ over $U$. Then $\alpha\left(\tilde{e}_{i}\right) \in \mathcal{O}_{X}(U)$ pulls back to a generator of $\mathcal{O}_{X_{j}}\left(-D_{i j}\right)$ on $U \times_{X} X_{j}$. Indeed this can be verified étale locally so it suffices to consider the case when $U$ is étale over

$$
\operatorname{Spec}\left(\kappa\left[x_{1}, \ldots, x_{n}\right] /\left(x_{1} \cdots x_{r}\right)\right)
$$

and $X_{i}$ (resp. $\left.X_{j}\right)$ is defined by the equation $x_{i}=0$ (resp. $x_{j}=0$ ). In this case it is clear that $\left(\alpha\left(\tilde{e}_{i}\right)\right)=\left(x_{i}\right)$. If $g: X_{j} \hookrightarrow X$ denotes the inclusion of $X_{j}$ in $X$, we therefore obtain a map of sheaves

$$
\left\{\text { liftings of } e_{i} \text { to } \mathcal{M}_{X}^{\mathrm{gp}}\right\} \longrightarrow g_{*}\left\{\text { trivializations of } \mathcal{O}_{X_{j}}\left(-D_{i j}\right)\right\},
$$

and this map is compatible with the natural action of $\mathcal{O}_{X}^{*}$ (where $\mathcal{O}_{X}^{*}$ acts on the right hand side through the map $\left.\mathcal{O}_{X}^{*} \rightarrow g_{*} \mathcal{O}_{X}^{*}\right)$. From this it follows that $\left.\mathcal{L}_{X_{i}}\right|_{X_{j}} \simeq \mathcal{O}_{X_{j}}\left(-D_{i j}\right)$.

Moreover, note that $\otimes_{i} \mathcal{L}_{X_{i}} \simeq \mathcal{O}_{X}$ since the image of 1 under the natural map $\mathbb{Z} \rightarrow \nu_{*} \mathbb{Z}$ lifts to $\mathcal{M}_{X}^{\prime g p}$. Indeed, the image of $1 \in \mathbb{N}$ under the map $\mathbb{N} \rightarrow \mathcal{M}_{X}^{\prime}$ obtained from the structure morphism (3.2.2) is such a lifting. Hence the restriction of $\mathcal{L}_{X_{i}}$ to $X_{i}$ is isomorphic to $\mathcal{O}_{X_{i}}\left(\sum_{j \neq i} D_{i j}\right)$.

\subsection{The degree map when $X$ is a curve.}

Though we do not need any results about the log Picard functor for curves, we include some basic observations and sketch an example to motivate the definition. 
Suppose all the components $X_{i}$ of $X$ have dimension 1 , and let $Y_{i} \subset \tilde{X}$ be the inverse image of $X_{i}$. For any line bundle $\mathcal{L}$ on $X$ define the degree of $\mathcal{L}$ by the formula

$$
\operatorname{deg}(\mathcal{L}):=\sum_{i} \operatorname{deg}_{Y_{i}}\left(\left.\nu^{*} \mathcal{L}\right|_{Y_{i}}\right)
$$

A corollary of the discussion (3.3) is the following:

Corollary 3.5. If $\mathcal{L}_{X_{i}}$ denotes the line bundle associated to a component $X_{i}$ as in (3.3), then $\operatorname{deg}\left(\mathcal{L}_{X_{i}}\right)=0$. Hence the degree map descends to a map

$$
H^{1}\left(X, \mathcal{M}_{X}^{\mathrm{gp}}\right) \longrightarrow \mathbb{Z}
$$

Proof. By (3.3),

$$
\operatorname{deg}\left(\mathcal{L}_{X_{i}}\right)=\operatorname{deg}_{Y_{i}}\left(\mathcal{O}_{Y_{i}}\left(\sum_{j \neq i} D_{i j}\right)\right)+\sum_{j \neq i} \operatorname{deg}_{Y_{j}}\left(\mathcal{O}_{Y_{j}}\left(-D_{i j}\right)\right)
$$

where $D_{i j}:=X_{i} \cap X_{j}$ (since $Y_{i} \rightarrow X_{i}$ is an isomorphism over $D_{i j}$, we also abusively view $D_{i j}$ as a subscheme of $Y_{i}$ ). Since

$$
\operatorname{deg}_{Y_{i}}\left(\mathcal{O}_{Y_{i}}\left(D_{i j}\right)\right)+\operatorname{deg}_{Y_{j}}\left(\mathcal{O}_{Y_{j}}\left(-D_{i j}\right)\right)=0
$$

the result follows.

Example 3.6. Suppose $X$ is the standard $N$-gon for some $N>2$ ([13], II.1.1). That is, $X$ is a chain of $\mathbb{P}^{1}$ 's glued together at 0 and $\infty$. By ([27], $\left.11.7(2)\right)$, there is a natural log structure $\mathcal{M}_{X}$ on $X$ and a special morphism from $\left(X, \mathcal{M}_{X}\right)$ to $\left(\operatorname{Spec}(\kappa), \mathcal{M}_{\kappa}\right)$, for some log structure $\mathcal{M}_{\kappa}$ on $\operatorname{Spec}(\kappa)$. Let $\operatorname{Pic}^{0}\left(X, \mathcal{M}_{X}\right)$ be the kernel of the map

$$
\operatorname{deg}: H^{1}\left(X, \mathcal{M}_{X}^{\mathrm{gp}}\right) \longrightarrow \mathbb{Z}
$$

Denote by $X_{\text {reg }} \subset X$ the smooth locus of $X$, and choose a point $e \in X_{\text {reg }}(\kappa)$. For any other point $t \in X_{\text {reg }}(\kappa)$, the sheaf $\mathcal{O}_{X}((t)-(e))$ defines a class in $\operatorname{Pic}^{0}\left(X, \mathcal{M}_{X}\right)$, and so we obtain a map

$$
X_{\mathrm{reg}}(\kappa) \longrightarrow \operatorname{Pic}^{0}\left(X, \mathcal{M}_{X}\right)
$$

which we claim is an isomorphism of groups.

To see this, let $\operatorname{Pic}^{[0]}(X)$ be the kernel of the degree map $\operatorname{Pic}(X) \rightarrow \mathbb{Z}$, and note that by ([13], II.3.4) there exists a surjective homomorphism

$$
\operatorname{Pic}^{[0]}(X) \longrightarrow X_{\text {reg }}(\kappa)
$$

with the property that $\mathcal{O}_{X}((t)-(e))$ maps to $t$. Denote by $K_{0}$ the kernel of (3.6.1). If $e_{i}$ denotes the point " 1 " on the $i$-th $\mathbb{P}^{1}\left(\right.$ so $\left.e=e_{0}\right)$. Then the elements $\mathcal{F}_{i}:=\mathcal{O}_{X}\left(\left(e_{i}\right)-\left(e_{0}\right)\right)$ $(i=1, \ldots N)$ map to a basis for

$$
\operatorname{Pic}^{[0]}(\tilde{X}):=\operatorname{Ker}(\operatorname{deg}: \operatorname{Pic}(\tilde{X}) \longrightarrow \mathbb{Z}) .
$$

and hence induce a natural exact sequence

$$
0 \longrightarrow \kappa^{*} \longrightarrow \mathrm{Pic}^{[0]}(X) \longrightarrow \oplus_{i=1}^{N-1} \mathbb{Z} \cdot \mathcal{F}_{i} \longrightarrow 0 .
$$


Since the composite of the inclusion $\kappa^{*} \hookrightarrow \operatorname{Pic}^{[0]}(X)$ with (3.6.1) is injective, the projection $K_{0} \rightarrow \mathbb{Z}^{N-1}$ is an inclusion. Moreover, there is a natural commutative diagram

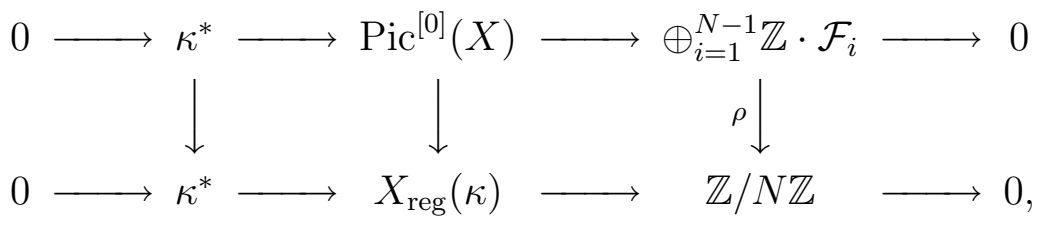

where $\rho$ is the map which sends $\mathcal{F}_{i}$ to $i$.

By (3.5), the degree of the $\mathcal{L}_{X_{i}}$ is zero, and we define $K_{1} \subset \mathrm{Pic}^{[0]}(X)$ to be the subgroup generated by the $\mathcal{L}_{X_{i}}$ so that we have an exact sequence

$$
0 \longrightarrow K_{1} \longrightarrow \operatorname{Pic}^{[0]}(X) \longrightarrow \operatorname{Pic}^{0}\left(X, \mathcal{M}_{X}\right) \longrightarrow 0 .
$$

To prove that (3.6.1) is an isomorphism it suffices to show that $K_{0}=K_{1}$. This follows from the following two lemmas whose proofs we leave to the reader.

Lemma 3.7. In $\operatorname{Pic}(X), \mathcal{L}_{X_{i}}=2 \mathcal{F}_{[i]}-\mathcal{F}_{[i+1]}-\mathcal{F}_{[i-1]}$, where for an integer $j$ we denote by $[j]$ the congruence class of $j$ modulo $N$ and $\mathcal{F}_{0}:=0$.

Lemma 3.8. The kernel of the map $\rho: \mathbb{Z}^{N-1} \rightarrow \mathbb{Z} / N \mathbb{Z}$ is generated by the elements $2 \mathcal{F}_{[i]}-$ $\mathcal{F}_{[i+1]}-\mathcal{F}_{[i-1]}$.

3.9. Intersection product when $X$ is a surface.

Suppose now that all components of $X$ have dimension 2, and let $\left\{Y_{i}\right\}$ be the connected components of the normalization $\nu: \tilde{X} \rightarrow X$ of $X$. On $\operatorname{Pic}\left(Y_{i}\right)$ we have the usual intersection pairing $<\cdot, \cdot>_{Y_{i}}$, and for two elements $\mathcal{L}_{1}, \mathcal{L}_{2} \in H^{1}\left(X, \mathcal{O}_{X}^{*}\right)$, we define

$$
<\mathcal{L}_{1}, \mathcal{L}_{2}>:=\sum_{i}<\left.\mathcal{L}_{1}\right|_{Y_{i}},\left.\mathcal{L}_{2}\right|_{Y_{i}}>_{Y_{i}} \in \mathbb{Z}
$$

The following proposition shows that this defines a pairing

$$
<\cdot, \cdot>: H^{1}\left(X, \mathcal{M}_{X}^{\mathrm{gp}}\right) \times H^{1}\left(X, \mathcal{M}_{X}^{\mathrm{gp}}\right) \longrightarrow \mathbb{Z} .
$$

Proposition 3.10. The pairing (3.9.1) descends to a pairing on $H^{1}\left(X, \mathcal{M}_{X}^{\mathrm{gp}}\right)$.

Proof. Let $G$ be the free abelian group on generators $\mathcal{L}_{X_{i}}$ corresponding to the components $X_{i}$ of $X$. The group $H^{1}\left(X, \mathcal{M}_{X}^{\text {gp }}\right)$ is the quotient of $H^{1}\left(X, \mathcal{O}_{X}^{*}\right)$ be the image of $G$ under the map (3.2.3). Thus since the pairing (3.9.1) is symmetric, it suffices to show that for every $\mathcal{L} \in H^{1}\left(X, \mathcal{O}_{X}^{*}\right)$,

$$
<\mathcal{L}_{X_{i}}, \mathcal{L}>=0
$$

Denote by $D_{i j}$ the intersection $\nu\left(X_{i}\right) \cap \nu\left(X_{j}\right)$. The curves $D_{i j}$ have at most nodal singularities, so it makes sense to speak of the degree $\operatorname{deg}_{D_{i j}}(\mathcal{L})$ of a line bundle on $D_{i j}$ ([13], I.3.3.4). Moreover, (3.3) shows that

$$
<\mathcal{L}_{X_{i}}, \mathcal{L}>=-\sum_{j \neq i} \operatorname{deg}_{D_{i j}}(\mathcal{L})-\sum_{j \neq i}<\left.\mathcal{L}_{X_{j}}\right|_{Y_{i}},\left.\mathcal{L}\right|_{Y_{i}}>_{Y_{i}}
$$

which equals

$$
-\sum_{j \neq i} \operatorname{deg}_{D_{i j}}(\mathcal{L})-\sum_{j \neq i}\left(-\operatorname{deg}_{D_{i j}}(\mathcal{L})\right)=0
$$




\section{The log Picard functor: general definition and representability}

4.1. Turning now to the general case, let

$$
\left(f, f^{b}\right):\left(X, \mathcal{M}_{X}\right) \rightarrow\left(S, \mathcal{M}_{S}\right)
$$

be a proper special log semi-stable morphism with $S$ a scheme. In this section we define a logarithmic Picard functor $\underline{\operatorname{Pic}}\left(\left(X, \mathcal{M}_{X}\right) /\left(S, \mathcal{M}_{S}\right)\right)$ and prove that it is representable by an algebraic space. In the case when $S=\operatorname{Spec}(\kappa)$ for a separably closed field $\kappa$, the set $\underline{\operatorname{Pic}}\left(\left(X, \mathcal{M}_{X}\right) /\left(S, \mathcal{M}_{S}\right)\right)(\kappa)$ will be in natural bijection with the group $H^{1}\left(X, \mathcal{M}_{X}^{\text {gp }}\right)$ discussed in the previous section.

Before defining the log Picard functor, however, it will be useful to consider the following stack:

Definition 4.2. The Picard stack of $\left(X, \mathcal{M}_{X}\right) /\left(S, \mathcal{M}_{S}\right)$, denoted $\mathcal{P} i c$, is the stack over the category of $S$-schemes whose fiber over any $g: T \rightarrow S$ is the groupoid of $\mathcal{M}_{X_{T}}^{\mathrm{gp}}$-torsors over $X_{T, \text { et }}$, where $\left(X_{T}, \mathcal{M}_{X_{T}}\right) \rightarrow\left(T, g^{*} \mathcal{M}_{S}\right)$ denotes the morphism obtained by base change.

Just as in the case of algebraic spaces ([5], p. 67), we need to impose a condition on (4.1.1) in order to understand the deformation theory of $\mathcal{P} i c$.

Definition 4.3. The morphism (4.1.1) is cohomologically flat in dimension 0 if for any nilpotent closed immersion $\operatorname{Spec}\left(A_{0}\right) \hookrightarrow \operatorname{Spec}(A)$ of affine schemes over $S$ defined by a squarezero ideal, the natural map

$$
H^{0}\left(X_{A}, \mathcal{M}_{X_{A}}^{\mathrm{gp}}\right) \longrightarrow H^{0}\left(X_{A_{0}}, \mathcal{M}_{X_{A_{0}}}^{\mathrm{gp}}\right)
$$

is surjective.

The following theorem is the main result of this section.

Theorem 4.4. If (4.1.1) is cohomologically flat in dimesion zero, then $\mathcal{P}$ ic is an algebraic stack locally of finite type over $S$.

Before giving the proof of (4.4), let us note some consequences pertaining to the log Picard functor.

Definition 4.5. For a special log semi-stable morphism as in (4.1.1) which is cohomologically flat in dimension 0, we define the log Picard functor, denoted $\operatorname{Pic}\left(\left(X, \mathcal{M}_{X}\right) /\left(S, \mathcal{M}_{S}\right)\right)$ (or just Pic if no confusion seems likely to arise), to be the sheaf associated to the presheaf which to any $T \rightarrow S$ associates the isomorphism classes of objects in $\operatorname{Pic}(T)$.

Corollary 4.6. The functor $\underline{\mathrm{Pic}}$ is representable by an algebraic space.

Proof. If $\lambda \in \mathcal{P} i c$ is an object over some $S$-scheme $T$, then the group of automorphisms of $\lambda$ in $\operatorname{Pic}(T)$ is the group $H^{0}\left(X_{T}, \mathcal{M}_{X_{T}}^{\mathrm{gp}}\right)$. Thus the corollary follows from our assumption of cohomological flatness in dimension 0 and the following general result.

Proposition 4.7. Let $y$ be an algebraic stack such that for any object $z: Z \rightarrow y$ over some scheme $Z$, the group algebraic space $\underline{\mathrm{Aut}}_{z}$ over $Z$ representing the functor of automorphisms of $z$ is smooth. Then the sheaf $Y$ associated to the presheaf which to any scheme $T$ associates the isomorphism classes of objects in $y(T)$ is representable by an algebraic space. 
Proof. This argument can be found in ([2], Appendix). We repeat it for completeness. Let $u: U \rightarrow y$ be a smooth cover, and let $V=U \times y, U, R=V \times_{U \times U} V$. Then $R$ defines an equivalence relation on $V$. Indeed to give a map $T \rightarrow V$ is equivalent to giving data $\left(t_{1}, t_{2}, \iota\right)$, where $t_{i}: T \rightarrow U$ are morphisms and $\iota: t_{1}^{*} u \simeq t_{2}^{*} u$ is an isomorphism in $y(T)$. $R$ is the sub-functor of $V \times V$ consisting of pairs of data

$$
\left\{\left(t_{1}, t_{2}, \iota\right),\left(t_{1}, t_{2}, \iota^{\prime}\right)\right\} \text {. }
$$

The fact that the Aut-functors are smooth implies that $R$ is a smooth equivalence relation on $V$. Denote by $\bar{V}=[V / R]$ the resulting algebraic space. The space $\bar{V}$ represents the functor over $U \times U$ which to any scheme $T$ associates $\{*\}$ if $\left.p_{1}^{*} u\right|_{T}$ and $\left.p_{2}^{*} u\right|_{T}$ are étale locally isomorphic and $\emptyset$ otherwise. Hence $\bar{V} \subset U \times U$ is the equivalence relation defining $Y$, and by construction it is a smooth equivalence relation. Hence $Y$ is representable by an algebraic space.

It will also be useful to define a log Picard functor for morphisms which are not special:

Definition 4.8. If $\left(f, f^{b}\right):\left(X, \mathcal{M}_{X}\right) \rightarrow\left(S, \mathcal{M}_{S}\right)$ is a (not necessarily special) proper $\log$ semi-stable morphism, we define the log Picard functor, denoted $\operatorname{Pic}\left(\left(X, \mathcal{M}_{X}\right) /\left(S, \mathcal{M}_{S}\right)\right.$ ) (or just $\underline{\mathrm{Pic}})$, to be the functor $\underline{\operatorname{Pic}}\left(\left(X, \mathcal{M}_{X}^{\sharp}\right) /\left(S, \mathcal{M}_{S}^{\sharp}\right)\right)$, where $\mathcal{M}_{X}^{\sharp}$ and $\mathcal{M}_{S}^{\sharp}$ are as in $(2.8)$.

In the rest of this section, we prove (4.4) by first reducing to the case when $S$ is an affine scheme of finite type over an excellent Dedekind ring, and then verifying the conditions of ([2], 5.3).

\subsection{Reduction to the case when $S$ is affine and of finite type over $\mathbb{Z}$.}

With out loss of generality, we can assume that $S=\operatorname{Spec}(A)$ is affine. Write $A$ as a filtering inductive limit $\lim _{\longrightarrow} A_{i}$ of $\mathbb{Z}$-algebras of finite type. Since $X / S$ is special, the stalks of $\overline{\mathcal{M}}_{S}$ are all isomorphic to $\overrightarrow{\mathbb{N}}^{r}$ for some $r$. By $([39], 5.14)$, there is an open substack $\mathcal{U} \subset \mathcal{L}$ og classifying such $\log$ structures, and since $\mathcal{U}$ is locally of finite presentation over $\mathbb{Z}$, we can approximate $\mathcal{M}_{S}$ by a $\log$ structure $\mathcal{M}_{A_{i}}$ on one of the $\operatorname{Spec}\left(A_{i}\right)$ for which the stalks of $\overline{\mathcal{M}}_{A_{i}}$ are all free monoids. Moreover, by ([39], 3.5) there exists an open substack $\mathcal{S} \subset \mathcal{L}_{\operatorname{og}}\left(\operatorname{Spec}\left(A_{i}\right), \mathcal{M}_{A_{i}}\right)$ classifying morphisms of $\log$ schemes $g: T \rightarrow\left(\operatorname{Spec}\left(A_{i}\right), \mathcal{M}_{A_{i}}\right)$ with the property that for every geometric point $\bar{t} \rightarrow \stackrel{\circ}{T}$ the map $\overline{\mathcal{M}}_{A_{i}, f(\bar{t})} \rightarrow \overline{\mathcal{M}}_{T, \bar{t}}$ is as in (2.10.1). Now since $\stackrel{\circ}{X} / \stackrel{\circ}{S}$ is of finite presentation, we can approximate $\stackrel{\circ}{X}$ by a proper scheme $f_{i}: \stackrel{\circ}{X}_{i} \rightarrow \operatorname{Spec}\left(A_{i}\right)$ after possibly increasing $i$, and since $\mathcal{S}$ is locally of finite presentation over $A_{i}$ we can by ([32], 4.18) also find an approximation $\mathcal{L}: \stackrel{\circ}{X}_{i} \rightarrow \mathcal{S}$ for the map $\stackrel{\circ}{X} \rightarrow \mathcal{S}$ induced by the $\log$ structure on $X$. Let $Z \subset \stackrel{\circ}{X}_{i}$ be the closed subset of points where $\mathcal{L}$ is not smooth. Since $f_{i}$ is proper, $f_{i}(Z) \subset \operatorname{Spec}\left(A_{i}\right)$ is a closed subset which does not meet the image of $\operatorname{Spec}(A) \rightarrow \operatorname{Spec}\left(A_{i}\right)$. Hence after increasing $i$, we can assume that $Z=\emptyset$ in which case the map $f_{i}: \stackrel{\circ}{X}_{i} \rightarrow \mathcal{S}$ is smooth. We have now found a proper log smooth morphism $X_{i} \rightarrow\left(\operatorname{Spec}\left(A_{i}\right), \mathcal{M}_{A_{i}}\right)$ inducing $X / S$. Moreover, for every geometric point $\bar{x} \rightarrow \stackrel{\circ}{X_{i}}$ in the singular locus, the map $\overline{\mathcal{M}}_{A_{i}, f(\bar{x})} \rightarrow \overline{\mathcal{M}}_{X_{i}, \bar{x}}$ is as in (2.10.1). From this and ([40], 2.2) it follows that $X_{i} \rightarrow\left(\operatorname{Spec}\left(A_{i}\right), \mathcal{M}_{A_{i}}\right)$ is a proper special log semi-stable morphism.

4.10. Pic is limit preserving. 
We have to show that if $\left\{A_{i}\right\}$ is a filtering inductive limit of noetherian rings over $S$, then the natural functor

$$
\lim _{\longrightarrow} \mathcal{P} i c\left(A_{i}\right) \longrightarrow \mathcal{P} i c\left(\lim _{\longrightarrow} A_{i}\right)
$$

is an equivalence of categories. To see that this functor is fully faithful, suppose given two objects $\lambda_{1}, \lambda_{2} \in \lim \mathcal{P} i c\left(A_{i}\right)$. After replacing $S$ by one of the $A_{i}$, we may assume that the $\lambda_{i}$ are induced by objects, which we also denote by $\lambda_{i}$, in $\operatorname{Pic}(S)$. Let $\operatorname{Spec}(R) \rightarrow X$ be an affine étale cover such that the restrictions of the $\lambda_{i}$ to $\operatorname{Spec}(R)$ are trivial, and let $\operatorname{Spec}\left(R^{\prime}\right)=$ $\operatorname{Spec}(R) \times_{X} \operatorname{Spec}(R)$. We then have a natural commutative diagram

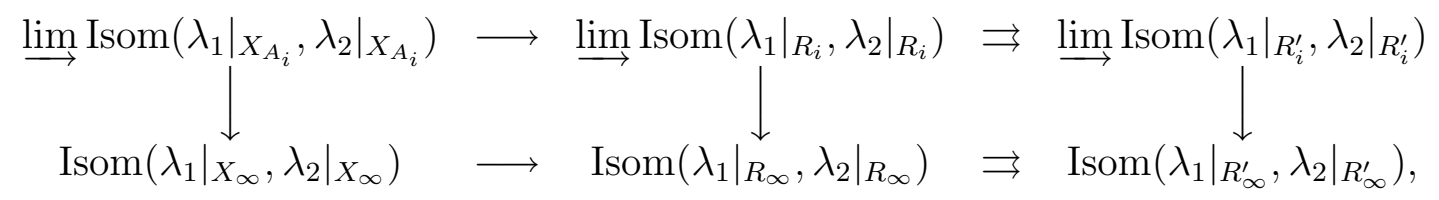

where $X_{A_{i}}$ (resp. $\left.R_{i}, R_{i}^{\prime}\right)$ denotes the base change of $X$ to $\operatorname{Spec}\left(A_{i}\right)\left(\right.$ resp. $\left.R \otimes_{\mathcal{O}_{S}} A_{i}, R^{\prime} \otimes_{\mathcal{O}_{S}} A_{i}\right)$ and $X_{\infty}$ (resp. $R_{\infty}, R_{\infty}^{\prime}$ ) denotes $\lim _{\longleftarrow} X_{A_{i}}$ (resp. $\underset{\lim }{\longrightarrow} R_{i}, \underset{\lim }{\longrightarrow} R_{i}^{\prime}$ ). Since the rows in $(4.10 .2)$ are exact, the following lemma proves the full faithfulness of $(4.10 .1)$.

Lemma 4.11. Let $\left\{R_{i}\right\}$ be a filtering inductive system of noetherian rings with compatible log structures $\mathcal{M}_{R_{i}}$. Then the natural map

$$
\lim H^{0}\left(\operatorname{Spec}\left(R_{i}\right), \mathcal{M}_{R_{i}}^{\mathrm{gp}}\right) \longrightarrow H^{0}\left(\operatorname{Spec}\left(R_{\infty}\right), \mathcal{M}_{R_{\infty}}^{\mathrm{gp}}\right)
$$

is an isomorphism, where $\mathcal{M}_{R_{\infty}}$ denotes the log structure on $\operatorname{Spec}\left(R_{\infty}\right)$ obtained from the $\mathcal{M}_{R_{i}}$.

Proof. From the exact sequences

$$
0 \longrightarrow \mathcal{O}_{R_{i}}^{*} \longrightarrow \mathcal{M}_{R_{i}}^{\mathrm{gp}} \longrightarrow \overline{\mathcal{M}}_{R_{i}}^{\mathrm{gp}} \longrightarrow 0
$$

we obtain a commutative diagram with exact columns

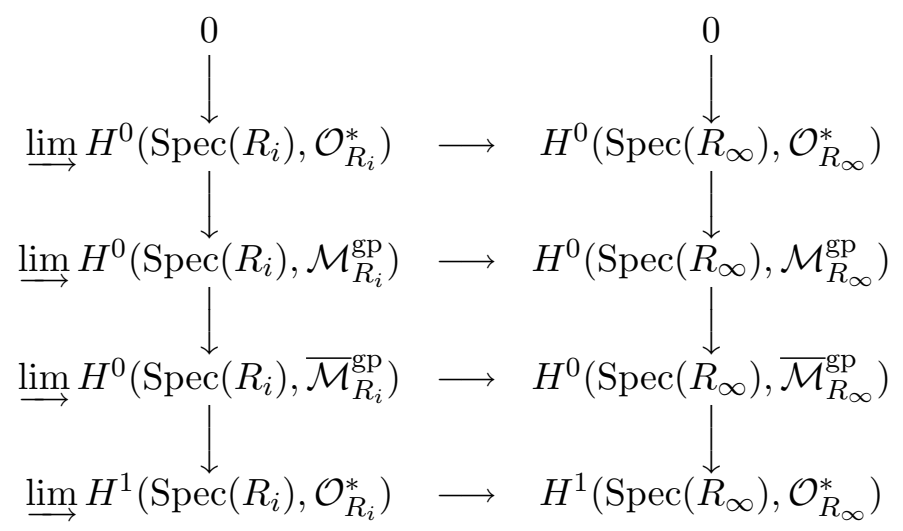

By standard properties of $\mathbb{G}_{m}$, the first and fourth horizontal arrows are isomorphisms, and by ([7], VII.5.7) the third horizontal arrow is an isomorphism also. Hence the second horizontal arrow is an isomorphism.

To see that (4.10.1) is essentially surjective, suppose given an object $\lambda_{\infty} \in \mathcal{P} i c\left(\underline{\lim } A_{i}\right)$. Let $\operatorname{Spec}\left(R_{\infty}\right) \rightarrow X_{\infty}$ be an affine étale cover for which $\left.\lambda_{\infty}\right|_{R_{\infty}}$ is trivial. After replacing $S$ by $\operatorname{Spec}\left(A_{i}\right)$ for $i$ sufficiently big, we can find an étale cover $\operatorname{Spec}(R) \rightarrow X$ such that $\operatorname{Spec}\left(R_{\infty}\right)=\operatorname{Spec}(R) \times_{X} X_{\infty}$. Let $\lambda_{R}$ denote the trivial torsor on $\operatorname{Spec}(R)$ and choose an 
isomorphism $\left.\left.\lambda_{R}\right|_{R_{\infty}} \simeq \lambda_{\infty}\right|_{R_{\infty}}$. By (4.11), we can after perhaps replacing $S$ by $\operatorname{Spec}\left(A_{i}\right)$ for an even bigger $i$, find an isomorphism $\sigma: p_{1}^{*} \lambda_{R} \simeq p_{2}^{*} \lambda_{R}$ over $\operatorname{Spec}(R) \times_{X} \operatorname{Spec}(R)$ inducing the tautological isomorphism $\left.\left.p_{1}^{*} \lambda_{\infty}\right|_{R_{\infty}} \simeq p_{2}^{*} \lambda_{\infty}\right|_{R_{\infty}} \operatorname{over} \operatorname{Spec}\left(R_{\infty}\right) \times_{X_{\infty}} \operatorname{Spec}\left(R_{\infty}\right)$. Moreover, by (4.11) the equality

$$
p_{13}^{*}(\sigma)=p_{23}^{*}(\sigma) \circ p_{12}^{*}(\sigma)
$$

holds over $\operatorname{Spec}(R) \times_{X} \operatorname{Spec}(R) \times_{X} \operatorname{Spec}(R)$ after possibly base changing to another $A_{i}$. In this case, the pair $\left(\lambda_{R}, \sigma\right)$ induces by descent an object $\lambda \in \operatorname{Pic}(X)$ whose image in $\operatorname{Pic}\left(X_{\infty}\right)$ is isomorphic to $\lambda_{\infty}$. Thus (4.10.1) is essentially surjective.

\subsection{Obstruction theory.}

Suppose $A \rightarrow A_{0}$ is a surjection of rings over $S$ with square-zero kernel $I$. Let

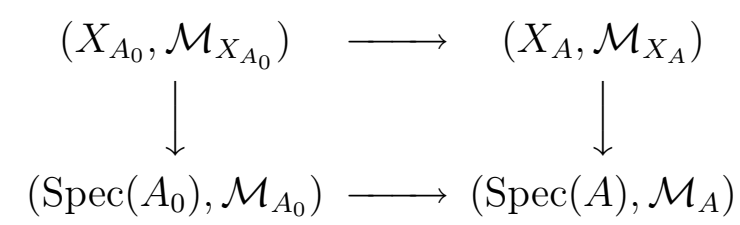

be the resulting diagram of log algebraic spaces. Suppose further that

$$
\lambda_{0} \in H^{1}\left(X_{A_{0}}, \mathcal{M}_{X_{A_{0}}}^{\mathrm{gp}}\right)
$$

is a class representing a torsor. Define a map

$$
\epsilon: I \otimes \mathcal{O}_{X_{A_{0}}} \longrightarrow \mathcal{M}_{X_{A}}^{\mathrm{gp}}
$$

by sending $i \otimes f$ to $1+i \tilde{f}$, where $\tilde{f}$ is a lifting of $f$ to $\mathcal{O}_{X_{A}}$. Then, as in the case of the usual exponential map ([5], p. 69), this does not depend on the choice of $\tilde{f}$ and is a homomorphism. Moreover, there is a natural exact sequence

$$
0 \longrightarrow I \otimes \mathcal{O}_{X_{A_{0}}} \stackrel{\epsilon}{\longrightarrow} \mathcal{M}_{X_{A}}^{\mathrm{gp}} \longrightarrow \mathcal{M}_{X_{A_{0}}}^{\mathrm{gp}} \longrightarrow 0
$$

from which it follows that the obstruction to lifting $\lambda_{0}$ to $X_{A}$ is a class

$$
o\left(\lambda_{0}\right) \in H^{2}\left(X_{A_{0}}, I \otimes \mathcal{O}_{X_{A_{0}}}\right) .
$$

In this way we obtain an obstruction theory, in the sense of $([2], 2.6)$, for $\mathcal{P} i c$. Moreover, the conditions $([2], 4.1)$ are satisfied by standard properties of cohomology.

\subsection{Schlessinger's conditions.}

Let us first make a general observation. If $A \rightarrow A_{0}$ is a surjective morphism with squarezero kernel $I$, then the sequence (4.12.1) gives rise to an exact sequence

$$
0 \longrightarrow H^{1}\left(X_{A_{0}}, I \otimes \mathcal{O}_{X_{A_{0}}}\right) \longrightarrow H^{1}\left(X_{A}, \mathcal{M}_{X_{A}}^{\mathrm{gp}}\right) \longrightarrow H^{1}\left(X_{A_{0}}, \mathcal{M}_{X_{A_{0}}}^{\mathrm{gp}}\right)
$$

by our assumption of cohomological flatness in dimension 0 . Thus given any $\lambda_{0} \in \mathcal{P} i c\left(X_{A_{0}}\right)$, the isomorphism classes of liftings of $\lambda_{0}$ to $X_{A}$ are naturally a torsor under $H^{1}\left(X_{A_{0}}, I \otimes \mathcal{O}_{X_{A_{0}}}\right)$.

Suppose now that

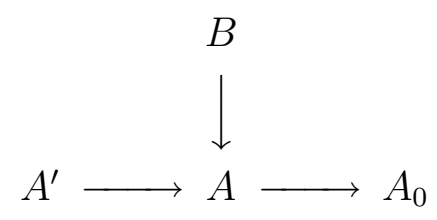


is a deformation situation as in ([2], 2.2), and fix $\lambda \in H^{1}\left(X_{A}, \mathcal{M}_{X_{A}}^{\mathrm{gp}}\right)$. Recall that $B \rightarrow A_{0}$ is surjective, $A \rightarrow A_{0}$ and $A^{\prime} \rightarrow A$ are surjective with nilpotent kernels, and $I:=\operatorname{Ker}\left(A^{\prime} \rightarrow A\right)$ is an $A_{0}$-module of finite type.

Proposition 4.14. The natural functor

$$
F: \mathcal{P} i c_{\lambda}\left(A^{\prime} \times_{A} B\right) \longrightarrow \mathcal{P} i c_{\lambda}\left(A^{\prime}\right) \times \mathcal{P} i c_{\lambda}(B)
$$

is an equivalence of categories. Here for any ring $R$ with a map $l: R \rightarrow A$, we denote by $\mathcal{P}_{i c_{\lambda}}(R)$ the category whose objects are pairs $\left(\lambda^{\prime}, \iota\right)$, where $\lambda^{\prime} \in \mathcal{P} i c(R)$ and $\iota: l^{*} \lambda^{\prime} \simeq \lambda$ is an isomorphism in $\mathcal{P} i c(A)$.

Proof. To see that $F$ is fully faithful, let $\lambda_{1}, \lambda_{2} \in \mathcal{P} i c_{\lambda}\left(A^{\prime} \times_{A} B\right)$ be two objects and consider the map

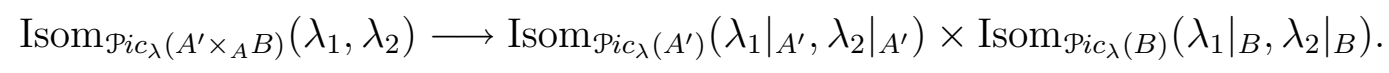

If the left hand side of (4.14.1) is non-empty, then the choice of an element in the set $\operatorname{Isom}_{\mathcal{P}_{i c_{\lambda}}\left(A^{\prime} \times{ }_{A} B\right)}\left(\lambda_{1}, \lambda_{2}\right)$ identifies the map (4.14.1) with the natural map

$$
\begin{gathered}
\operatorname{Ker}\left(H^{0}\left(X_{A^{\prime} \times_{A} B}, \mathcal{M}_{X_{A^{\prime} \times A_{A} B}}^{\mathrm{gp}}\right) \rightarrow H^{0}\left(X_{A}, \mathcal{M}_{X_{A}}^{\mathrm{gp}}\right)\right) \\
\operatorname{Ker}\left(H^{0}\left(X_{A^{\prime}}, \mathcal{M}_{X_{A^{\prime} \times_{A} B}}^{\mathrm{gp}}\right) \rightarrow H^{0}\left(X_{A}, \mathcal{M}_{X_{A}}^{\mathrm{gp}}\right)\right) \oplus \operatorname{Ker}\left(H^{0}\left(X_{B}, \mathcal{M}_{X_{B}}^{\mathrm{gp}}\right) \rightarrow H^{0}\left(X_{A}, \mathcal{M}_{X_{A}}^{\mathrm{gp}}\right)\right) .
\end{gathered}
$$

Since the kernel of

$$
\operatorname{Ker}\left(H^{0}\left(X_{A^{\prime} \times_{A} B}, \mathcal{M}_{X_{A^{\prime} \times A_{B}}}^{\mathrm{gp}}\right) \rightarrow H^{0}\left(X_{A}, \mathcal{M}_{X_{A}}^{\mathrm{gp}}\right)\right) \longrightarrow \operatorname{Ker}\left(H^{0}\left(X_{B}, \mathcal{M}_{X_{B}}^{\mathrm{gp}}\right) \rightarrow H^{0}\left(X_{A}, \mathcal{M}_{X_{A}}^{\mathrm{gp}}\right)\right)
$$

is by the exponential sequence (4.12.1) isomorphic to $H^{0}\left(X_{A}, I \otimes \mathcal{O}_{X_{A_{0}}}\right)$ as is

$$
\operatorname{Ker}\left(H^{0}\left(X_{A^{\prime}}, \mathcal{M}_{X_{A^{\prime}}}^{\mathrm{gp}}\right) \rightarrow H^{0}\left(X_{A}, \mathcal{M}_{X_{A}}^{\mathrm{gp}}\right)\right),
$$

the map (4.14.2) is an isomorphism. Hence to prove that (4.14.1) is bijective it suffices to show that the left hand side is non-empty if there exists an isomorphism

$$
\left(\iota_{1}:\left.\left.\lambda_{1}\right|_{A^{\prime}} \rightarrow \lambda_{2}\right|_{A^{\prime}}, \iota_{2}:\left.\left.\lambda_{1}\right|_{B} \rightarrow \lambda_{2}\right|_{B}\right)
$$

in $\mathcal{P} i c_{\lambda}\left(A^{\prime}\right) \times \mathcal{P} i c_{\lambda}(B)$.

To see this, view $\lambda_{1}$ as a lifting of $\left.\lambda_{2}\right|_{B}$ via $\iota_{2}$. Then the obstruction to lifting $\iota_{2}$ to $A^{\prime} \times_{A} B$ lies in $H^{1}\left(X_{A_{0}}, I \otimes \mathcal{O}_{X_{A_{0}}}\right)$, and is equal to the class defined by the liftings $\left.\lambda_{1}\right|_{A^{\prime}}$ and $\left.\lambda_{2}\right|_{A^{\prime}}$. Hence this class is zero, and we can find an isomorphism $\iota: \lambda_{1} \simeq \lambda_{2}$ inducing $\iota_{2}$. Thus $F$ is fully faithful.

To see that $F$ is essentially surjective, let $\left(\lambda_{1}, \lambda_{2}\right) \in \mathcal{P} i c_{\lambda}\left(A^{\prime}\right) \times \mathcal{P} i c_{\lambda}(B)$ be an object. Then the obstruction to lifting $\lambda_{2}$ to $X_{A^{\prime} \times_{A} B}$ lies in $H^{2}\left(X_{A_{0}}, I \otimes \mathcal{O}_{X_{A_{0}}}\right)$, and this obstruction is by functoriality equal to the obstruction to lifting $\lambda$ to $X_{A^{\prime}}$. Hence $\lambda_{2}$ lifts. Choose such a lifting $\tilde{\lambda}$. Then $\left.\tilde{\lambda}\right|_{X_{A^{\prime}}}$ is isomorphic to $\lambda_{1}$ in $\mathcal{P} i c_{\lambda}\left(A^{\prime}\right)$ if and only if the corresponding class $c \in H^{1}\left(X_{A_{0}}, I \otimes \mathcal{O}_{X_{A_{0}}}\right)$ is zero. But as mentioned above, the isomorphism classes of liftings of $\lambda_{2}$ is canonically in a torsor under this same group. From this we conclude that there exists a unique lifting $\tilde{\lambda} \in \mathcal{P} i c_{\lambda}\left(A^{\prime} \times_{A} B\right)$ of $\left(\lambda_{1}, \lambda_{2}\right)$, and hence $F$ is an equivalence of categories. 
From this and standard properties of cohomology we conclude that conditions 1 and 3 of ([2], 5.3) hold.

\subsection{Quasi-separation condition.}

We have to show that if $A_{0}$ is a noetherian integral domain over $S$, and if $\lambda \in \mathcal{P} i c\left(A_{0}\right)$ admits an automorphism $\sigma \in H^{0}\left(X_{A_{0}}, \mathcal{M}_{X_{A_{0}}}^{\mathrm{gp}}\right)$ which induces the identity in $\operatorname{Pic}(\kappa)$ for a dense set of points $A_{0} \rightarrow \kappa$ of finite type, then there exists an open dense subset of $\operatorname{Spec}\left(A_{0}\right)$ over which $\sigma$ is the identity. By ([39], 3.5 (ii) and (iii)), the set of points $x \in X_{A_{0}}$ for which the image of $\sigma$ in $\overline{\mathcal{M}}_{X_{A_{0}, \bar{x}}}^{\mathrm{gp}}$ is zero is an open set. Let $Z \subset X_{A_{0}}$ be its complement. Since $X_{A_{0}} / A_{0}$ is proper, the image of $Z$ in $\operatorname{Spec}\left(A_{0}\right)$ is a closed set whose complement contains a dense set of points. Hence after shrinking $\operatorname{Spec}\left(A_{0}\right)$, we may assume that $\sigma \in H^{0}\left(X_{A_{0}}, \mathcal{O}_{X_{A_{0}}}^{*}\right)$. Since $X_{A_{0}}$ is flat over $A_{0}$ by $([28], 4.5)$, the open set $Z(\sigma-1)^{c} \subset X_{A_{0}}$ maps to an open set in $\operatorname{Spec}\left(A_{0}\right)$ whose complement is dense by assumption. Hence $Z(\sigma-1)=X_{A_{0}}$, and since $X_{A_{0}}$ is reduced we must have $\sigma=1$.

\subsection{Compatibility with completion.}

By $([2], 5.3)$ the following proposition completes the proof of $(4.4)$.

Proposition 4.17. Let $\hat{A}$ be a complete noetherian local ring with separably closed residue field $\kappa$ and maximal ideal $\mathfrak{m}$, and let $\hat{A}_{n}=\hat{A} / \mathfrak{m}^{n+1}$. Then the natural functor

is an equivalence of categories.

$$
\operatorname{Pic}(\hat{A}) \longrightarrow \lim \operatorname{P} i c\left(\hat{A}_{n}\right)
$$

Proof. The proof of (4.17) occupies the remainder of this section. Let

$$
\overline{\mathcal{M}}_{X_{\hat{A}}}^{\mathrm{gp}} \simeq \bigoplus_{i \in \mathcal{C}\left(X_{A_{0}}\right)} \overline{\mathcal{M}}_{i}^{\mathrm{gp}}
$$

be the decomposition given in (2.12).

Lemma 4.18. For each $i \in \mathcal{C}\left(X_{A_{0}}\right)$, the natural map

$$
H^{0}\left(X_{\hat{A}}, \overline{\mathcal{M}}_{i}^{\mathrm{gp}}\right) \longrightarrow \lim _{\longleftarrow} H^{0}\left(X_{\hat{A}_{n}}, \overline{\mathcal{M}}_{i, n}^{\mathrm{gp}}\right)
$$

is an isomorphism, and

$$
H^{1}\left(X_{\hat{A}}, \overline{\mathcal{M}}_{i}^{\mathrm{gp}}\right)=0=H^{1}\left(X_{\hat{A}_{n}}, \overline{\mathcal{M}}_{i, n}^{\mathrm{gp}}\right)
$$

for all $n$.

Proof. Let $Z_{i} \subset X_{\hat{A}}$ be the connected component of the singular locus corresponding to $i \in \mathcal{C}\left(X_{A_{0}}\right)$. By $(2.12)$ there is a canonical isomorphism $H^{0}\left(\operatorname{Spec}(\hat{A}), \overline{\mathcal{M}}_{\operatorname{Spec}(\hat{A})}\right) \simeq \mathbb{N}^{\mathcal{C}\left(X_{A_{0}}\right)}$, where $\mathcal{M}_{\text {Spec }(\hat{A})}$ denotes the pullback of the log structure on $S$. Let $t_{i} \in A$ be the image in $\hat{A}$ of a lifting of the $i$-th standard generator of $\mathbb{N}^{\mathcal{C}\left(X_{A_{0}}\right)}$ to $\mathcal{M}_{\operatorname{Spec}(\hat{A})}$. The element $t_{i} \in \hat{A}$ is well defined up to a unit. Since the sheaf $\overline{\mathcal{M}}_{i}^{\text {gp }}$ has support on the closed subscheme of $X_{\hat{A}}$ 
defined by $t_{i}=0$, we can replace $A$ by $A /\left(t_{i}\right)$, and hence can assume that $t_{i}=0$. In this case, we can find an étale neighborhood of the form

$$
\hat{A}\left[x_{1}, \ldots, x_{n}\right] /\left(x_{1} \cdots x_{r}\right)
$$

around any point $z \in Z_{i}$, and the ideal $J_{i}$ of $Z_{i}$ is equal to

$$
\left(x_{1} \cdots \widehat{x}_{j} \cdots x_{r}\right)_{j=1}^{r} \text {. }
$$

Let $\nu_{i}: \tilde{X}_{i} \rightarrow X$ be the blow-up of $J_{i}$. Then by (2.15) the sheaf $\overline{\mathcal{M}}_{i}^{\text {gp }}$ is isomorphic to $\nu_{i *} \mathbb{Z}$. Thus $H^{0}\left(X_{\hat{A}}, \overline{\mathcal{M}}_{i}^{\text {gp }}\right)$ is isomorphic to a direct sum of $\mathbb{Z}$ 's indexed by the connected components of $\tilde{X}_{i}$, and similarly for the reductions. Thus the first isomorphism follows from ([4], 3.1) which implies that the connected components of $\tilde{X}_{i}$ are in bijection with the connected components of the reductions of $\tilde{X}_{i}$. The statement about the $H^{1}$ 's follows from ([7], IX.3.6).

Define $K\left(\operatorname{resp} . K_{n}\right)$ to be the image of

$$
H^{0}\left(X_{\hat{A}}, \mathcal{M}_{X_{\hat{A}}}^{\mathrm{gp}}\right) \longrightarrow H^{0}\left(X_{\hat{A}}, \overline{\mathcal{M}}_{X_{\hat{A}}}^{\mathrm{gp}}\right) \quad\left(\text { resp. } H^{0}\left(X_{\hat{A}_{n}}, \mathcal{M}_{X_{\hat{A}_{n}}}^{\mathrm{gp}}\right) \longrightarrow H^{0}\left(X_{\hat{A}_{n}}, \overline{\mathcal{M}}_{X_{\hat{A}_{n}}}^{\mathrm{gp}}\right)\right)
$$

and let $Q$ (resp. $Q_{n}$ ) be the cokernel. Our assumption of cohomological flatness implies that the natural maps $K_{n} \rightarrow K_{n-1}$ are all isomorphisms, and hence there is a morphism of exact sequences

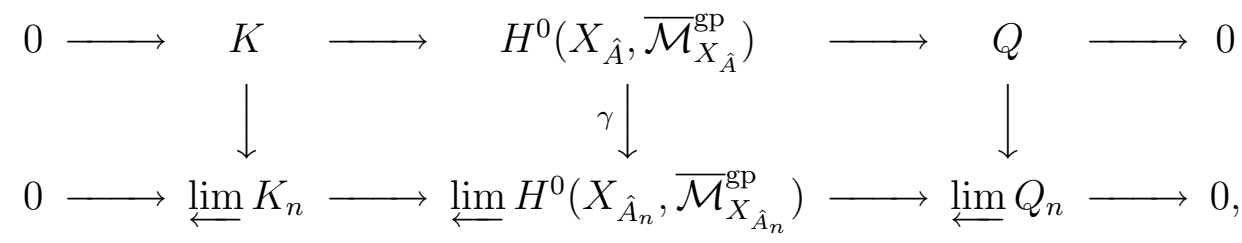

where the map labeled $\gamma$ is an isomorphism by (4.18).

Lemma 4.19. Suppose $Y / \hat{A}$ is a proper algebraic space which has the property that for any surjective morphism $R \rightarrow R_{0}$ of $\hat{A}$-algebras with square-zero kernel, the natural map

$$
H^{0}\left(Y_{R}, \mathcal{O}_{Y_{R}}^{*}\right) \longrightarrow H^{0}\left(Y_{R_{0}}, \mathcal{O}_{Y_{R_{0}}}^{*}\right)
$$

is surjective. Then the natural map

$$
H^{1}\left(Y, \mathcal{O}_{Y}^{*}\right) \longrightarrow \lim ^{1} H^{1}\left(Y_{n}, \mathcal{O}_{Y_{n}}^{*}\right)
$$

is an isomorphism, where $Y_{n}$ denotes the reduction of $Y$ modulo $\mathfrak{m}^{n+1}$.

Proof. The Grothendieck existence theorem ([14], III.5.1.6) implies that to give an invertible sheaf $\mathcal{L}$ on $Y$ is equivalent to giving a compatible family of invertible sheaves $\left\{\mathcal{L}_{n}\right\}$ on the reductions. From this it follows that it is enough to show that if $\left\{\mathcal{L}_{n}\right\}$ and $\left\{\mathcal{L}_{n}^{\prime}\right\}$ are two compatible families such that for all $n, \mathcal{L}_{n}$ and $\mathcal{L}_{n}^{\prime}$ are isomorphic, then in fact the two families are isomorphic. For this it is enough to show that any automorphism of some $\mathcal{L}_{n}$ can be lifted to $\mathcal{L}_{n+1}$. In other words, that the map

$$
H^{0}\left(Y_{n+1}, \mathcal{O}_{Y_{n+1}}^{*}\right) \longrightarrow H^{0}\left(Y_{n}, \mathcal{O}_{Y_{n}}^{*}\right)
$$

is surjective. 
Note that the lemma applies to spaces obtained by pullback from $X / S$. Indeed if

$$
\operatorname{Spec}\left(R_{0}\right) \hookrightarrow \operatorname{Spec}(R)
$$

is a closed immersion over $S$ defined by a square zero ideal in $R$, then the map

$$
H^{0}\left(X_{R}, \mathcal{M}_{X_{R}}^{\mathrm{gp}}\right) \longrightarrow H^{0}\left(X_{R_{0}}, \mathcal{M}_{X_{R_{0}}}^{\mathrm{gp}}\right)
$$

is surjective by the assumption of cohomological flatness, and since the diagram of sheaves on $X_{R}$

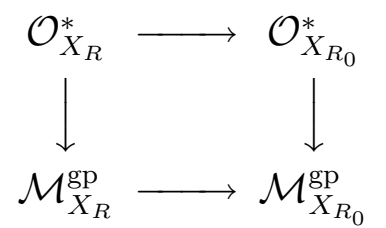

is cartesian by the definition of a log structure it follows that the map

$$
H^{0}\left(X_{R}, \mathcal{O}_{X_{R}}^{*}\right) \longrightarrow H^{0}\left(X_{R_{0}}, \mathcal{O}_{X_{R_{0}}}^{*}\right)
$$

is surjective.

Lemma 4.20. (i) The natural maps

$$
K \longrightarrow \lim _{\longleftarrow} K_{n}, \quad Q \longrightarrow \lim _{\longleftarrow} Q_{n}
$$

are isomorphisms.

(ii) The maps

$$
H^{0}\left(X_{\hat{A}}, \mathcal{M}_{X_{\hat{A}}}^{\mathrm{gp}}\right) \longrightarrow \lim _{\longleftarrow} H^{0}\left(X_{n}, \mathcal{M}_{X_{n}}^{\mathrm{gp}}\right), \quad H^{1}\left(X_{\hat{A}}, \mathcal{M}_{X_{\hat{A}}}^{\mathrm{gp}}\right) \longrightarrow \lim _{\longleftarrow} H^{1}\left(X_{n}, \mathcal{M}_{X_{n}}^{\mathrm{gp}}\right)
$$

are isomorphisms.

Proof. To see (i), note that it follows from (4.19) that the map $Q \rightarrow \underset{\lim }{\longleftarrow} Q_{n}$ is injective since there is a natural commutative square

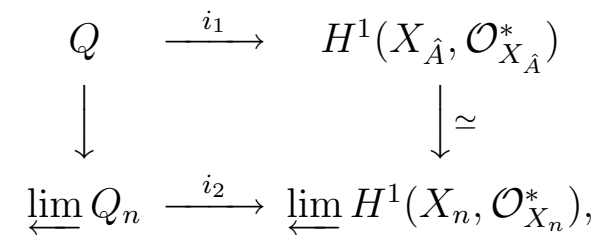

where the maps $i_{1}$ and $i_{2}$ are injective. Combining this with the five lemma applied to (4.18.1), it follows that the maps (4.20.1) are isomorphisms.

Statement (ii) follows from (i) and (4.19) by considering the natural morphisms of exact sequences

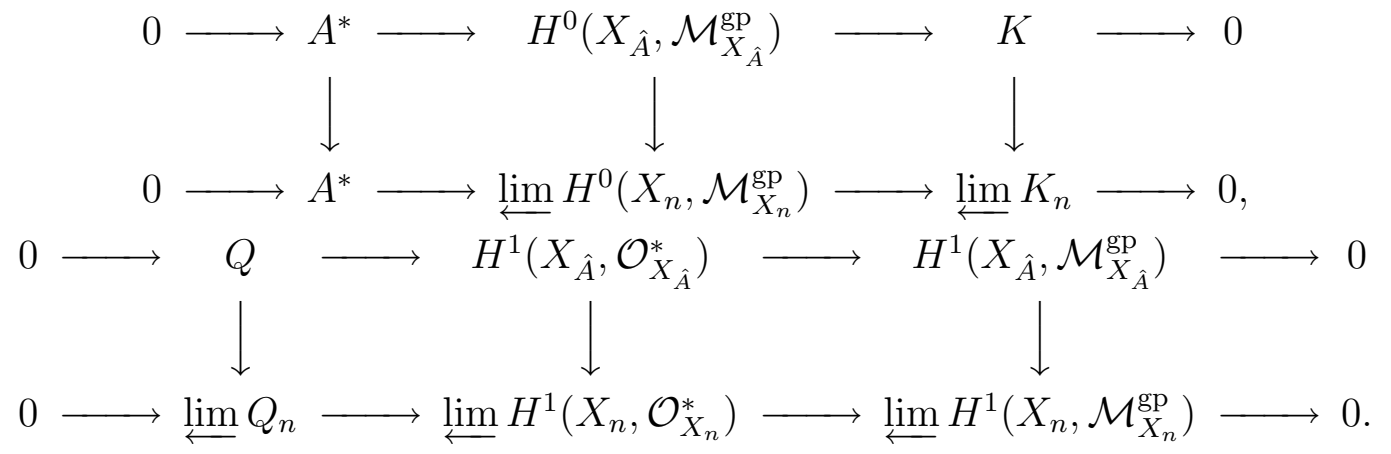


4.21 (Completion of proof of (4.17)). Suppose given a compatible family $\left\{\lambda_{n}\right\}_{n} \in \lim _{\longleftarrow} \mathcal{P} i c\left(\hat{A}_{n}\right)$. By the second isomorphism in (4.20.2) we can find an object $\lambda \in \mathcal{P} i c(\hat{A})$ whose reduction $\bmod \mathfrak{m}^{n+1}$ is isomorphic to $\lambda_{n}$ for each $n$. We claim that in fact we can choose a compatible family of isomorphisms. It suffices to show that any isomorphism $\iota_{n}:\left.\lambda\right|_{\hat{A}_{n}} \rightarrow \lambda_{n}$ can be lifted to an isomorphism $\iota_{n+1}:\left.\lambda\right|_{\hat{A}_{n+1}} \rightarrow \lambda_{n+1}$. By assumption there exists an isomorphism $\tilde{\iota}:\left.\lambda\right|_{\hat{A}_{n+1}} \rightarrow \lambda_{n+1}$, and choosing one such isomorphism we obtain an automorphism $\left.\iota_{n} \circ \tilde{\iota}\right|_{\hat{A}_{n}}$

of $\left.\lambda\right|_{\hat{A}_{n}}$. Now by our assumption of cohomological flatness this automorphism can be lifted to $\hat{A}_{n+1}$ and twisting $\tilde{\iota}$ by a lift we obtain $\iota_{n+1}$. This proves that the functor is essentially surjective.

To see that is it fully faithful, observe that the second isomorphism in (4.20.2) combined with the argument of the preceding paragraph show that two elements $\lambda_{1}, \lambda_{2} \in \operatorname{Pic}(\hat{A})$ map to isomorphic objects in $\lim _{\longleftarrow} \operatorname{P} i c\left(\hat{A}_{n}\right)$ if and only if $\left.\left.\lambda_{1}\right|_{\hat{A}_{n}} \simeq \lambda_{2}\right|_{\hat{A}_{n}}$ for every $n$. This combined with the first isomorphism in (4.20.2) implies that the functor is fully faithful.

\section{Polarized log K3 surfaces}

Definition 5.1 (cf. [29] or [35]). A morphism of $\log$ algebraic spaces $f: X \rightarrow S$ is a $\log$ $K 3$ surface if it is proper, log semi-stable, and if for all geometric points $\bar{s} \rightarrow \stackrel{\circ}{S}$ the second exterior power $\Omega_{X_{\bar{s}}}^{2}$ of the log differentials is isomorphic to $\mathcal{O}_{X_{\bar{s}}}$ and $H^{1}\left(X_{\bar{s}}, \mathcal{O}_{X_{\bar{s}}}\right)=0$. A log $\mathrm{K} 3$ surface $X / S$ is special if the morphism $f$ is special in the sense of (2.7).

Remark 5.2. If $X / S$ is a $\log \mathrm{K} 3$ surface and $\stackrel{\circ}{S}$ is isomorphic to the spectrum of an algebraically closed field, then $\stackrel{\circ}{X}$ is a scheme. This follows from ([24], appendix B.3.4), which shows that the normalization of each component of $\stackrel{\circ}{X}$ is a scheme, combined with (5.13) and (5.14 (i)) below.

Remark 5.3. It follows from the argument of $([35], 3.4)$ that the underlying algebraic space of a $\log \mathrm{K} 3$ surface X over a separably closed field is a combinatorial K3 surface in the sense of (1.1).

Definition 5.4. A log K3 surface $X / S$ is stable if the geometric fibers of $\stackrel{\circ}{X} / \stackrel{\circ}{S}$ are all stable in the sense of (1.1).

Proposition 5.5. Let $X / S$ be a stable log K3 surface with $\stackrel{\circ}{S} \simeq \operatorname{Spec}(\kappa)$ for some field $\kappa$ of characteristic 0 . Then the Hodge numbers $h^{p q}=\operatorname{dim}_{\kappa} H^{q}\left(X, \Omega_{X}^{p}\right)$ are as follows:

$$
\begin{array}{ccc}
1 & 0 & 1 \\
0 & 20 & 0 \\
1 & 0 & 1
\end{array}
$$

Proof. Since $\kappa$ has characteristic 0, one can reduce in a standard way to the case when $\kappa=\mathbb{C}$. In this case the result follows from ([29], 2.5 and 4.1). 
Corollary 5.6. If $f: X \rightarrow S$ is a $\log K 3$ surface, then $f$ is cohomologically flat in dimension 0 , and hence by (4.6) the associated logarithmic Picard functor Pic is representable by an algebraic space.

Definition 5.7. Let $X / S$ be a log K3 surface, and let $\underline{\text { Pic }}$ be the associated Picard space

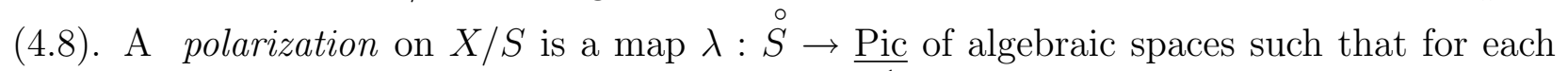
geometric fiber $X_{\bar{s}}$, there exists a lifting $\mathcal{L}$ of $\lambda_{\bar{s}}$ to $\overline{H^{1}}\left(X_{\bar{s}}, \mathcal{O}_{X_{\bar{s}}}^{*}\right)$ such that for some $N>0$ the sheaf $\mathcal{L}^{N}$ is generated by global sections and the map $X_{\bar{s}} \rightarrow \bar{X}_{\bar{s}} \subset \mathbb{P}^{r}$ (for some $r$ ) defined by $\mathcal{L}^{N}$ contracts only finitely many curves on $X_{\bar{s}}$ to points. $\lambda$ is primitive if for every geometric point $\bar{s} \rightarrow \stackrel{\circ}{S}$, the corresponding element $\lambda_{\bar{s}} \in \underline{\operatorname{Pic}}(\bar{s})$ is a primitive element.

Remark 5.8. Here $\underline{\operatorname{Pic}}(\bar{s})$ has a group structure from the isomorphism

$$
\underline{\operatorname{Pic}}(\bar{s}) \simeq H^{1}\left(X_{\bar{s}}, \mathcal{M}_{X_{\bar{s}}}^{\sharp, g p}\right. \text {. }
$$

5.9. Let $\kappa$ be an algebraically closed field of characteristic 0 , and let $X /\left(\operatorname{Spec}(\kappa), \mathcal{M}_{\kappa}\right)$ be a special stable $\log \mathrm{K} 3$ surface (where $\operatorname{Spec}(\kappa)$ has the $\log$ structure associated to $\mathbb{N} \rightarrow \kappa$, $1 \mapsto 0)$. Assume given a line bundle $\mathcal{L}$ on $X$ which is generated by global sections and let $\pi: X \rightarrow \bar{X} \subset \mathbb{P}^{r}$ (for some $r>0$ ) be the map to projective space defined by $\mathcal{L}$. Here $\bar{X}$ denotes the scheme-theoretic image of $X$. We assume that $\pi$ only contracts finitely many curves on $X$ to points (so in particular $\pi$ restricted to each component of $X$ defines a birational map onto its image). The following technical result will play an important role in what follows:

Theorem 5.10. For $N$ sufficiently big, the map $\pi: X \rightarrow \bar{X}$ defined by $\mathcal{L}^{N}$ is étale locally on $\bar{X}$ relatively projective.

The proof occupies the remainder of this section.

Let us begin with some general remarks about contractions. Recall the following result due to Artin:

Theorem 5.11 ([3], 6.1). Let $X$ be an algebraic space, $Y \subset X$ a closed subspace and $Y \rightarrow Y^{\prime}$ a finite morphism. Then the coproduct $\bar{X}:=X \coprod_{Y} Y^{\prime}$ in the category of algebraic spaces exists and is the unique maximal modification of $X$ along $Y \rightarrow Y^{\prime}$ (see ([3], p. 88) for the definition of a modification). Moreover, if $U \rightarrow \bar{X}$ is étale, then $U=X_{U} \bigsqcup_{Y_{U}} Y_{U^{\prime}}$ (where $X_{U}=X \times{ }_{\bar{X}} U$ etc.).

5.12. The proof of (5.11) also provides a description of the completion of the local ring of $\bar{X}$ at a geometric point. It shows that the formal completion of $\bar{X}$ along $Y^{\prime}$ can be described as follows. Let $\operatorname{Spec}\left(A^{\prime}\right) \rightarrow Y^{\prime}$ be an étale morphism and let $\operatorname{Spec}(A) \rightarrow Y$ be the base change to $Y$. Because of the invariance of the étale site under infinitesimal thickenings ([23], I.8.3), we can lift $A$ to an étale map of formal schemes $\operatorname{Spf}(\tilde{A}) \rightarrow \widehat{X}$, where $\widehat{X}$ denotes the formal completion of $X$ along $Y$. Then the proof of (5.11) shows that the formal completion of $\bar{X}$ along $Y^{\prime}$ is étale locally isomorphic to $\operatorname{Spf}\left(\tilde{A} \times_{A} A^{\prime}\right)$. Moreover, this étale local description of the formal completion of $\bar{X}$ along $Y^{\prime}$ completely characterizes $\bar{X}$ by ([3], 3.1). The uniqueness part of (loc. cit.) also implies that if $X=\operatorname{Spec}(R), Y=\operatorname{Spec}(A)$ and $Y^{\prime}=\operatorname{Spec}\left(A^{\prime}\right)$ are all affine then

$$
X \coprod_{Y} Y^{\prime}=\operatorname{Spec}\left(R \times_{A} A^{\prime}\right)
$$


Lemma 5.13. Let $X$ and $Y$ be schemes of finite type over an algebraically closed field $\kappa$ and $j_{1} \coprod j_{2}: Y \coprod Y \hookrightarrow X$ a closed immersion. Suppose that for every point $p \in Y$ there exists an affine open $U \subset X$ containing the two points $j_{i}(p)(i=1,2)$. Then the coproduct $X \coprod_{Y} \amalg_{Y} Y$ in the category of algebraic spaces is a scheme.

Proof. Let $\bar{X}$ be the ringed space $\left(|X| \coprod_{|Y| \amalg|Y|}|Y|, g_{*} \mathcal{O}_{X} \times_{g_{*} \mathcal{O}_{Y} \amalg Y} h_{*} \mathcal{O}_{Y}\right)$, where $g:|X| \rightarrow$ $|\bar{X}|$ and $h:|Y| \rightarrow|\bar{X}|$ denote the natural morphisms of topological spaces. We claim that $\bar{X}$ is a scheme, and that the natural map of ringed spaces $X \rightarrow \bar{X}$ induces a map of formal schemes as in (5.12). This will prove the lemma by the uniqueness part of ([3], 3.1). Let $p \in Y$ be a point, and let $\operatorname{Spec}(A) \subset Y$ be an affine neighborhood of $p$. Then it follows from our assumptions that we can find an affine open $\operatorname{Spec}(B) \subset X$ containing the points $j_{i}(p)$ such that $\operatorname{Spec}(B) \cap(Y \coprod Y) \subset \operatorname{Spec}(A \times A)$. Let $f \in A$ be an element such that $D(f \times f) \subset \operatorname{Spec}(A \times A)$ is contained in $\operatorname{Spec}(B) \cap(Y \coprod Y)$, and let $g \in B$ be a lifting of $f \times f$. Then replacing $B$ by $B_{g}$ and $A$ by $A_{f}$, we see that we can find an affine open neighborhood $\operatorname{Spec}(A) \subset Y$ of $p$ and an open set $\operatorname{Spec}(B) \subset X$ such that $\operatorname{Spec}(B) \cap(Y \coprod Y)=\operatorname{Spec}(A \times A)$. In this case, $\operatorname{Spec}\left(B \times_{A \times A} A\right)$ defines an open neighborhood of $p$ in $\bar{X}$ which is a scheme. Hence $\bar{X}$ is a scheme, and the above local description of $\bar{X}$ proves that the associated map of formal schemes has the desired properties.

Lemma 5.14. (i) Let $X$ be a semi-stable surface over an algebraically closed field $\kappa$, and let $\tilde{X}$ be the normalization of $X$. Let $Y \subset X$ be the singular locus (with the reduced structure) and let $\tilde{Y} \subset \tilde{X}$ be the inverse image of $Y$. Then the natural map

$$
\tilde{X} \coprod_{\tilde{Y}} Y \longrightarrow X
$$

is an isomorphism.

(ii) Let $V$ be a smooth surface (possibly disconnected) over an algebraically closed field $\kappa$ and let $Y / \kappa$ be a smooth curve. Suppose given an inclusion

$$
j_{1} \coprod j_{2}: \tilde{Y}:=Y \coprod Y \hookrightarrow V
$$

such that each inclusion $j_{i}: Y \subset V$ identifies $Y$ with a smooth divisor on $V$. Then the algebraic space $\bar{V}:=V \coprod_{\tilde{Y}} Y$ is semi-stable.

Proof. To see (i), note that the question is étale local on $X$, so we may assume that $X=$ $\operatorname{Spec}(\kappa[x, y, z] /(x y$ or $x y z))$. In this case it follows that the map (5.14.1) is the one induced by the isomorphism

$$
\kappa[x, y, z] /(x y) \longrightarrow \kappa[x, z] \times_{\kappa[z]} \kappa[y, z],
$$

or

$\kappa[x, y, z] /(x y z) \longrightarrow(\kappa[x, y] \times \kappa[y, z] \times \kappa[x, z]) \times_{(\kappa[x, y] /(x y) \times \kappa[y, z] /(y z) \times \kappa[x, z] /(x z))} \kappa[x, y, z] /(x y, y z, x z)$.

As for (ii), it suffices to look at the formal completion of $\bar{V}$ at a point $P \in Y$. Since $Y$ is smooth, we can find an étale neighborhood $\left(\operatorname{Spec}(R), P^{\prime}\right) \rightarrow(Y, P)$ of $P$ together with an étale morphism $\operatorname{Spec}(R) \rightarrow \operatorname{Spec}(\kappa[t])$ sending $P^{\prime}$ to the origin. Let $A_{i}(i=1,2)$ be the unique liftings of $R$ to formal étale neighborhoods in $V$ of the preimages $P_{i}(i=1,2)$ of $P$ in $V$, where $\operatorname{Spec}(R)$ is viewed as an étale neighborhood of $P_{i} \in Y$ via the inclusion 
$j_{i}: Y \hookrightarrow V$. Then by (5.12), the formal completion of $\bar{X}$ at $P$ is isomorphic to the completion of $\left(A_{1} \times A_{2}\right) \times_{R \times R} R$. Since $V$ is smooth, we can find étale maps

$$
\kappa[t][[x]] \longrightarrow A_{1}, \quad \kappa[t][[y]] \longrightarrow A_{2}
$$

reducing to the specified map $\kappa[t] \rightarrow R$ (send $t$ to a lifting of $t \in R$ and send $x$ and $y$ to elements such that the image of $(t, x)$ and $(t, y)$ generate the ideals of $P_{1}$ and $P_{2}$ respectively). Then by ([41], 3.2.2) the induced map

$$
\kappa[t][[x, y]] /(x y) \simeq(\kappa[t][[x]] \times \kappa[t][[y]]) \times_{\kappa[t] \times \kappa[t]} \kappa[t] \longrightarrow\left(A_{1} \times A_{2}\right) \times_{R \times R} R
$$

is étale and hence induces an isomorphism on completions.

With these technicalities we can prove (5.10) treating in turn each of the different possibilities for the fibers as described by the following definition and result of Shephard-Barron.

Definition 5.15 ([44], p. 145). Let $V$ be an irreducible component of $X$ and let $C \subset V$ be an irreducible curve. $C$ is called a

(i) (§)-curve if $C$ is isomorphic to $\mathbb{P}^{1}$, is disjoint from all double curves, and $\left.C\right|_{\tilde{V}} ^{2}=-2$.

(ii) ( $\dagger$ )-curve if $C$ is isomorphic to $\mathbb{P}^{1}$, meets the locus of double curves in exactly one point which is not a triple point, and $\left.C\right|_{\tilde{V}} ^{2}=-1$.

(iii) (*)-curve if $C$ is a double curve (possibly equal to a curve of self-intersection of $V$ ) such that $C \simeq \mathbb{P}^{1}$ and $C^{2}=-1$ on each irreducible component on which it lies (in the case when $C$ equals a curve of self-intersection of $V$ this means that each of the two connected components of $\left.C\right|_{\tilde{V}} \subset \tilde{V}$ has self-intersection -1).

Proposition 5.16 ([44], 2.7). Let $V$ be an irreducible component of $X$ and let $\mathcal{C}=\left\{C_{1}, \ldots, C_{r}\right\}$ be a connected configuration of curves contracted by $\pi$ on $V$. Then $\mathcal{C}$ is of one of the following forms:

(i) $\mathcal{C}=\{C\}$ where $C$ is a $\left(^{*}\right)$-curve.

(ii) $C_{1}$ is a (†)-curve and $C_{2}, \ldots, C_{r}$ are $(\S)$-curve such that the dual graph of $\mathcal{C}$ is the following:

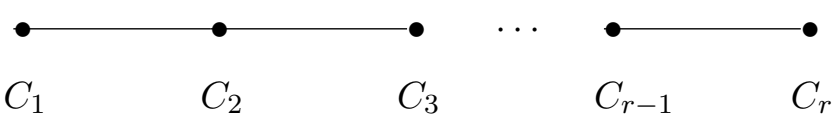

(iii) $\mathcal{C}$ is a negative definite configuration of $(\S)$-curves.

Lemma 5.17. For $N$ sufficiently big, the map $\pi$ has connected fibers.

Proof. We can without loss of generality assume $\kappa=\mathbb{C}$. By $([20], 5.5)$ and $([16], 4.3)$, there exists a smoothing $(\tilde{X}, \tilde{\mathcal{L}})$ of the pair $(\stackrel{\circ}{X}, \mathcal{L})$ over the disc $\Delta$ such that for $N$ suitably big $\tilde{\mathcal{L}}^{N}$ is generated by global sections. Let $\bar{f}: \stackrel{\circ}{X} \rightarrow \bar{X} \subset \mathbb{P}_{\Delta}^{r}$ be the image of the morphism to projective space defined by $\tilde{\mathcal{L}}^{N}$. To prove the lemma, it suffices by the analytic version of Zariski's main theorem ([22], p. 213) to show that for suitably big $N$ the space $\bar{X}$ is normal. To verify this we may make a finite base change $\Delta \rightarrow \Delta$ as in ([44], 2.10) and perform permissible elementary modifications in the sense of (loc. cit., page 145) by (loc. cit., 2.1). Thus the result follows from (loc. cit, proof of theorem $2 \mathrm{~W}$ ). 
For the rest of the proof we assume that $N$ is chosen so that $\pi$ has connected fibers. Since the assertion of (5.10) is étale local on $\bar{X}$, we can without loss of generality assume that $\bar{X}=\operatorname{Spec}(A)$ and that $\pi: X \rightarrow \bar{X}$ is an isomorphism away from some point $P \in \bar{X}$. We consider the various possibilities for $\pi^{-1}(P)$.

5.18. $\pi^{-1}(P)=\{C\}$ is a $\left({ }^{*}\right)$-curve.

Proof. It follows from the "triple point formula" ([19], p. 8) that $C$ cannot be a nodal curve lying in the intersection of a smooth component and a component with self-intersection. This implies that there exists a component $U$ of $X$ which meets $C$ but does not contain $C$. Let $\mathcal{L}_{U}$ be the line bundle on $X$ corresponding to the component $U$ (3.3). Since $C$ is rational and $\left.\mathcal{L}_{U}\right|_{C} \simeq \mathcal{O}_{C}(U \cap C), \mathcal{L}_{U}$ defines a relatively ample invertible sheaf in some neighborhood of $P$ by ([14], III.4.7.1).

5.19. $\pi^{-1}(P)$ is a negative definite configuration of (§)-curves.

Proof. Since every smooth proper surface over $\kappa$ is projective ([24], appendix B.3.4), the map $X \rightarrow \bar{X}$ is Zariski locally on $\bar{X}$ a proper morphism between quasi-projective schemes, and hence is a blow-up by ([14], III.2.3.7).

5.20. $\pi^{-1}(P)$ meets two smooth components and the restriction of $\pi^{-1}(P)$ to each component is a configuration as in (5.16 (ii)).

Proof. Let $V$ and $U$ be the two components of $X$ and let $\left\{C_{1}, \ldots, C_{r}\right\}$ (resp. $\left\{C_{1}^{\prime}, \ldots, C_{s}^{\prime}\right\}$ ) be the curves on $U$ (resp. $V$ ) contracted by $\pi$. Let $Y \subset V \cap U$ be the locus of intersection. We can then contract the curves $\left\{C_{1}, \ldots, C_{r}\right\}$ on $V$ to get a smooth space $V^{\prime}$ (first contract $C_{1}$, then $C_{2}$ etc. ) and similarly contract $\left\{C_{1}^{\prime}, \ldots, C_{s}^{\prime}\right\}$ to give a space $U^{\prime}$. Then the strict transforms of $Y$ in $V^{\prime}$ and $U^{\prime}$ are isomorphic to $Y$, and we can form the space $X^{\prime}=V^{\prime} \coprod_{Y} U^{\prime}$. By the universal property of coproduct we obtain a map

$$
X^{\prime} \rightarrow \bar{X}
$$

It suffices to show that the map $X \rightarrow X^{\prime}$ obtained from (5.14 (i)) is projective. For then (5.20.1) is proper, and since it is bijective on points it is an isomorphism.

We prove by induction on $r+s$ that the map $X \rightarrow X^{\prime}$ is projective. So consider first the case when $r=1$ and $s=0$. Let $V^{\prime}$ be the space obtained by contracting $C_{1}$ on $V$, and let $P \in X^{\prime}$ be the point which is the image of $C_{1}$. By assumption $X^{\prime}$ is quasi-projective. Thus there exists an effective Cartier divisor $D$ on $U$ such that $\operatorname{ord}_{P}(D)=1$ and such that $D$ meets $Y$ transversally. We view $D$ as a Cartier divisor on $X^{\prime}$. We claim that there is a natural closed immersion $X \hookrightarrow \mathbb{B}$, where $\mathbb{B}$ denotes the blow-up of $X^{\prime}$ along $D$. By the universal property of blow-up, this assertion is étale local on $X^{\prime}$. Since $X^{\prime}$ is semi-stable by (5.14 (ii)), we may assume that $X^{\prime}=\operatorname{Spec}(\kappa[x, y, x] /(x y))$ and that $P$ is the point $(x, y, z)$. We can further assume that $V^{\prime}$ is the component $y=0, U$ is the component $x=0$, and $D$ is the closed subscheme defined by $(x, z)$. Then $V$ is the blow-up of $V^{\prime}$ at $P$ and it is elementary to check the assertion. This proves the case when $r+s=1$. To get the general case, assume without loss of generality that $r \geq 1$. Then contract $C_{1}$ on $V$ to get $V^{\prime}$ and let $X^{\prime}=V^{\prime} \coprod_{Y} U$. Then by induction $X^{\prime}$ is projective over $\bar{X}$ and the case $r+s=1$ shows that $X \rightarrow X^{\prime}$ is projective. 
5.21. Case 4: $\pi^{-1}(P)$ contains a $(\dagger)$-curve and $X$ is irreducible.

Proof. By shrinking $X$, we may assume that the singular locus $Y \subset X$ is smooth. Let $\tilde{X}$ be the normalization of $X$ and let $\tilde{Y} \subset \tilde{X}$ be the inverse image of $Y$ (so $\tilde{Y} \simeq Y \coprod Y$ ). The inverse image of $P$ in $\tilde{X}$ then consists of two connected components $\left\{C_{1}, \ldots, C_{r}\right\}$ and $\left\{C_{1}^{\prime}, \ldots, C_{s}^{\prime}\right\}$ as in $\left(5.16\right.$ (ii)). Let $\tilde{X}_{1}$ be the space obtained by contracting the configuration $\left\{C_{1}, \ldots, C_{r}\right\}$ on $\tilde{X}$ and let $X_{1}=\tilde{X}_{1} \coprod_{\tilde{Y}} Y$. By $\left(5.14\right.$ (ii)), the space $X_{1}$ is semi-stable. We claim that if $s \geq 1$, then the map $X \rightarrow X_{1}$ is projective.

In the case when $s \geq 1, C_{1}^{\prime}$ defines a divisor on $X_{1}$ whose restriction to $Y$ is equal to the divisor of $P$. The map $\tilde{X} \rightarrow \tilde{X}_{1}$ is the blow-up of a sheaf $\tilde{J}$ on $\tilde{X}_{1}$ with support equal to one of the points in the inverse image of $P([24]$, ex. 7.11 (c)). We denote the subscheme of $\tilde{X}_{1}$ defined by $\tilde{J}$ by $Z_{1}$. Let $r$ be the order of vanishing of $\tilde{J}$ at $P$, and let $r C_{1}^{\prime} \subset \tilde{X}_{1}$ be the closed subscheme defined by $r$ times the ideal of $C_{1}^{\prime}$. If $Z \subset Y$ denotes the closed subscheme corresponding to the divisor $r P$, then we let $W:=\left(Z_{1} \coprod_{r} C_{1}^{\prime}\right) \coprod_{Z}{ }_{Z} Z$. By construction there is a natural map $W \rightarrow X_{1}$. We claim that this map is a closed immersion and that if $\mathbb{B}$ denotes the blow-up of $X_{1}$ along $W$, then there is a natural closed immersion $X \hookrightarrow \mathbb{B}$.

These assertions can all be verified étale locally on $X_{1}$. Thus we may assume that $X_{1}$ is étale over $\operatorname{Spec}(\kappa[x, y, z] /(x y))$ and that $X_{1}$ is affine, say equal to $\operatorname{Spec}(A)$. We can further assume that $C_{1}^{\prime}$ is defined by the ideal $(y, z)$. Let $A_{1}=A /(x), A_{2}=A /(y)$, and let $B=A /(x, y)$. Since $A$ is étale over $\kappa[x, y, z] /(x y)$, it follows that

$$
A=\left(A_{1} \times A_{2}\right) \times_{B \times B} B, \quad \tilde{A}=A_{1} \times A_{2} .
$$

The ideal $\tilde{J}$ is then an ideal of $A_{1}$ such that $\tilde{J}$ induces the ideal $\left(z^{r}\right)$ in $B$. W is the spectrum of

$$
\left(\left(A_{1} / \tilde{J}\right) \times A_{2} /\left(z^{r}\right)\right) \times_{B /\left(z^{r}\right) \times B /\left(z^{r}\right)} B /\left(z^{r}\right) .
$$

From this it is clear that the map $W \rightarrow X_{1}$ is a closed immersion, and that $W$ is defined by the ideal

$$
I=\left(\tilde{J} \times\left(z^{r}\right)\right) \times\left(z^{r}\right) \times\left(z^{r}\right)\left(z^{r}\right) .
$$

From this description it also follows that the natural projection map $I \rightarrow \tilde{J}$ is surjective as is the map $I \rightarrow\left(z^{r}\right)$. This implies that there is a natural closed immersion $X \hookrightarrow \mathbb{B}$.

To complete the proof of this case, it therefore suffices to consider the case when $s=0$. We construct $X_{1}$ as in the preceding paragraphs. Then the map $X_{1} \rightarrow \bar{X}$ is proper and injective; hence an isomorphism. Thus $\bar{X}$ is semi-stable by (5.14 (ii)). Replacing $\bar{X}$ by an étale cover we may therefore assume that $\bar{X}=\operatorname{Spec}(A)$ is affine and étale over $\operatorname{Spec}(\kappa[x, y, z] /(x y))$. The argument now proceeds as in the preceding paragraph using the ideal $(z)$ instead of $C_{1}^{\prime}$.

\section{The stack of polarized log K3 surfaces}

6.1. Fix an integer $k \geq 1$, and let $\mathbb{M}_{2 k} / \mathbb{Q}$ be the fibered category whose fiber over a scheme $T$ is the groupoid of triples $\left(\mathcal{M}_{T}, X, \lambda\right)$, where $\mathcal{M}_{T}$ is a log structure on $T, X /\left(T, \mathcal{M}_{T}\right)$ is a special stable log K3 surface, and $\lambda$ is a primitive polarization on $X /\left(T, \mathcal{M}_{T}\right)$ such that for each geometric point $\bar{t} \rightarrow T$ the restriction $\lambda_{\bar{t}}$ of $\lambda$ to $X_{\bar{t}}$ satisfies $\lambda_{\bar{t}}^{2}=2 k$. A morphism

$$
\left(\mathcal{M}_{T}, X, \lambda\right) \rightarrow\left(\mathcal{M}_{T}^{\prime}, X^{\prime}, \lambda^{\prime}\right)
$$


consists of a pair $(\iota, \gamma)$, where $\iota: \mathcal{M}_{T}^{\prime} \rightarrow \mathcal{M}_{T}$ is an isomorphism of $\log$ structures on $T$ and $\gamma: X \rightarrow X^{\prime}$ is an isomorphism of log algebraic spaces such that the diagram

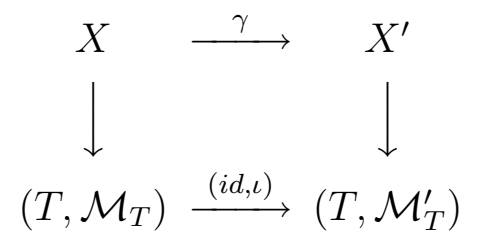

commutes, and such that $\gamma$ is compatible with the polarizations. We denote by

$$
\mathcal{M}_{\mathbb{M}_{2 k}}: \mathbb{M}_{2 k} \longrightarrow \mathcal{L} \log _{\left(\operatorname{Spec}(\mathbb{Q}), \mathbb{Q}^{*}\right)}
$$

the functor $\left(\mathcal{M}_{T}, X, \lambda\right) \mapsto \mathcal{M}_{T}$.

Theorem 6.2. $\mathbb{M}_{2 k}$ is a smooth Deligne-Mumford stack. There is a natural open substack $\mathbb{M}_{2 k}^{\mathrm{sm}} \subset \mathbb{M}_{2 k}$ classifying smooth (in the usual sense) polarized $\mathrm{K} 3$ surfaces, and the complement $\mathbb{M}_{2 k}-\mathbb{M}_{2 k}^{\mathrm{sm}}$ is a smooth divisor. Moreover, the functor $\mathcal{M}_{\mathbb{M}_{2 k}}$ is that induced by the log structure associated to the divisor $\mathbb{M}_{2 k}-\mathbb{M}_{2 k}^{\mathrm{sm}}$.

Remark 6.3. Before proving the theorem, let us remark how the stack $\mathbb{M}_{2 k}$ viewed as a log stack (B.1) with log structure $\mathcal{M}_{\mathbb{M}_{2 k}}$ coming from the divisor at infinity can be interpreted as a solution to a moduli problem on the category $F \log$ of fine $\log$ schemes over $\mathbb{Q}$. If $T$ is a fine $\log$ scheme over $\mathbb{Q}$, then we define $\left(\mathbb{M}_{2 k}, \mathcal{M}_{\mathbb{M}_{2 k}}\right)(T)$ to be the groupoid of morphisms of log stacks $T \rightarrow\left(\mathbb{M}_{2 k}, \mathcal{M}_{\mathbb{M}_{2 k}}\right)$ (B.3). There is a natural notion of pullback, so this defines a fibered category over F Log. Theorem (2.5) and (2.8) imply that this fibered category is equivalent to the fibered category whose fiber over $T$ is the groupoid of pairs $(X, \lambda)$, where $X / T$ is a $\log \mathrm{K} 3$ surface and $\lambda: \stackrel{\circ}{T} \rightarrow \underline{\mathrm{Pic}}(X / T)$ is a map of algebraic spaces such that for every geometric point $\bar{t} \rightarrow \stackrel{\circ}{T}$ the element $\lambda_{\bar{t}} \in H^{1}\left(X_{\bar{t}}, \mathcal{M}_{X_{\bar{t}}}^{\mathrm{gp}}\right)$ is a primitive polarization of degree $2 k$. If we give $F \log$ the topology where coverings are given by strict étale morphisms, then $\left(\mathbb{M}_{2 k}, \mathcal{M}_{\mathbb{M}_{2 k}}\right)$ is even a stack.

We prove the theorem by verifying the conditions of (B.12).

6.4. $\mathbb{M}_{2 k}$ is limit preserving.

Suppose $\left\{A_{i}\right\}_{i \in I}$ is a filtering inductive system of noetherian rings and consider the functor

$$
\stackrel{\lim }{\longrightarrow} \mathbb{M}_{2 k}\left(A_{i}\right) \longrightarrow \mathbb{M}_{2 k}\left(A_{\infty}\right),
$$

where $A_{\infty}:=\underline{\lim } A_{i}$. To see that (6.4.1) is fully faithful, suppose given two objects $\left(\mathcal{M}_{A_{i}}^{\ell}, X_{i}^{\ell}, \lambda_{i}^{\ell}\right)$ $(\ell=1,2)$ over some $\operatorname{Spec}\left(A_{i}\right)$. For each morphism $A_{i} \rightarrow A_{j}$, let $\left(\mathcal{M}_{A_{j}}^{\ell}, X_{j}^{\ell}, \lambda_{j}^{\ell}\right)$ be the data obtained by base change, and let $\left(\mathcal{M}_{A_{\infty}}^{\ell}, X_{\infty}^{\ell}, \lambda_{\infty}^{\ell}\right)$ be the induced data over $A_{\infty}$. We have to show that the map

$\underset{j}{\lim } \operatorname{Isom}_{\mathbb{M}_{2 k}\left(A_{j}\right)}\left(\left(\mathcal{M}_{A_{j}}^{1}, X_{j}^{1}, \lambda_{j}^{1}\right),\left(\mathcal{M}_{A_{j}}^{2}, X_{j}^{2}, \lambda_{j}^{2}\right)\right) \rightarrow \operatorname{Isom}_{\mathbb{M}_{2 k}\left(A_{\infty}\right)}\left(\left(\mathcal{M}_{A_{\infty}}^{1}, X_{\infty}^{1}, \lambda_{\infty}^{1}\right),\left(\mathcal{M}_{A_{\infty}}^{2}, X_{\infty}^{2}, \lambda_{\infty}^{2}\right)\right)$

is bijective. Suppose first that $\left(\iota_{\ell}, \gamma_{\ell}\right)(\ell=1,2)$ are two isomorphisms over some $A_{j}$ which become equal in the limit. Then since the stack $\mathcal{L} o g$ is limit preserving (it is locally of finite type over $\operatorname{Spec}(\mathbb{Z})$ by $([39], 1.1)$ ), we can after passing to some sufficiently big $j$ assume that $\iota_{1}=\iota_{2}$. Moreover, since the spaces $\stackrel{\circ}{j}_{j}^{\ell}$ are of finite presentation over $A_{j}$, we can by a standard 
limit assume that $\stackrel{\circ}{\gamma}_{1}=\stackrel{\circ}{2}_{2}$, after passing to some even bigger $j$. In this case it follows from (2.9) that $\gamma_{1}=\gamma_{2}$. Hence (6.4.2) is injective.

To see that (6.4.2) is surjective, suppose $(\iota, \gamma)$ is an isomorphism over $A_{\infty}$. Once again since $\mathcal{L} o g$ is limit preserving and $X_{\infty}^{\ell} / A_{\infty}$ is of finite presentation, we can approximate $\left(\iota, \gamma^{\circ}\right)$ be some $\left(\iota_{j}, \stackrel{\circ}{\gamma}_{j}\right)$ over some $A_{j}$. Then $\left(\stackrel{\circ}{X_{j}^{1}}, \stackrel{\circ}{\gamma}_{j}^{*} \mathcal{M}_{X_{j}^{2}}\right)$ and $X_{j}^{1}$ are two proper special semi-stable log schemes over $\left(\operatorname{Spec}\left(A_{j}\right), \mathcal{M}_{A_{j}}^{1}\right)$ and hence by $(2.5)$ and $(2.9)$ the pair $\left(\iota_{j}, \stackrel{\circ}{\gamma}_{j}\right)$ extends uniquely to an isomorphism $\left(\iota_{j}, \gamma_{j}\right)$ inducing $(\iota, \gamma)$. Moreover, since Pic is limit preserving, it follows that for some sufficiently big $j$ the isomorphism $\left(\iota_{j}, \gamma_{j}\right)$ is compatible with the polarizations, and hence (6.4.2) is bijective.

To see that (6.4.1) is essentially surjective, let $\left(\mathcal{M}_{A_{\infty}}, X, \lambda\right)$ be an object over $A_{\infty}$. Since $\stackrel{\circ}{X} / A_{\infty}$ is of finite presentation, we can approximate $\stackrel{\circ}{X}$ by some proper space $\stackrel{\circ}{X}_{j} / A_{j}$ for some $j$. Moreover, since $\stackrel{\circ}{X}$ is étale locally isomorphic to

$$
A_{\infty}\left[x_{1}, \ldots, x_{r}\right] /\left(x_{1} \cdots x_{l}-t\right)
$$

for some $t \in A_{\infty}$, one shows by a standard limit argument that after increasing $j$, we can assume that $\stackrel{\circ}{X}_{j} / A_{j}$ is proper and semi-stable. In this case we can define the functor IVLS ${ }_{X_{j} / A_{j}}$, and since this functor is of finite type over $A_{j}$ there exists for some sufficiently big $j$ an element in IVLS $_{\AA_{X_{j} / A_{j}}}$ inducing the morphism of $\log$ schemes $X \rightarrow\left(\operatorname{Spec}\left(A_{\infty}\right), \mathcal{M}_{A_{\infty}}\right)$. Moreover, since

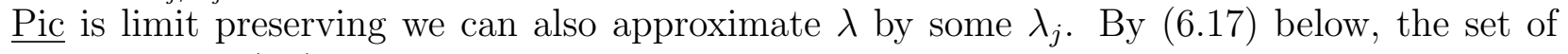
points in $\operatorname{Spec}\left(A_{j}\right)$ where $\lambda_{j}$ is a primitive polarization of degree $2 k$ is an open set, and hence after passing to some bigger $j$ we obtain the desired object $\left(\mathcal{M}_{A_{j}}, X_{j}, \lambda_{j}\right)$ over some $A_{j}$.

6.5. Schlessinger's conditions $\left(\mathrm{S} 1^{\log }\right)$ and $\left(\mathrm{S} 2^{\log }\right)$.

Lemma 6.6. For any logarithmic deformation situation as in (B.6), and log smooth, integral morphism $X \rightarrow \operatorname{Spec}(\mathcal{A})$, the natural functor

$\left(\log\right.$ smooth liftings of $X$ to $\left.\mathcal{A}^{\prime} \times{ }_{\mathcal{A}} \mathcal{B}\right)$

\section{$\downarrow$ \\ (log smooth liftings of $X$ to $\left.\mathcal{A}^{\prime}\right) \times(\log$ smooth liftings of $X$ to $\mathcal{B})$}

is an equivalence of categories.

Proof. It suffices to prove the following: for any $\log$ smooth lifting $X_{\mathcal{B}}$ of $X$ to $\mathcal{B}$ the natural functor

(6.6.1) $\quad\left(\log\right.$ smooth liftings of $X_{\mathcal{B}}$ to $\left.\mathcal{A}^{\prime} \times{ }_{\mathcal{A}} \mathcal{B}\right) \longrightarrow\left(\log\right.$ smooth liftings of $X$ to $\left.\mathcal{A}^{\prime}\right)$

is an equivalence of categories. Now this statement is étale local on $X_{\mathcal{B}}$, so it suffices to consider the case when $X_{\mathcal{B}}$ is affine. In this case both categories in (6.6.1) are non-empty and groupoids ([28], $3.14(1)$ ), so it suffices to show that for any lifting $\tilde{X}$ of $X_{\mathcal{B}}$ inducing a lifting $\tilde{X}_{\mathcal{A}^{\prime}}$ of $X$, the map

$$
\operatorname{Aut}_{X_{\mathcal{B}}}(\tilde{X}) \longrightarrow \operatorname{Aut}_{X}\left(\tilde{X}_{\mathcal{A}^{\prime}}\right)
$$


is bijective. But by ([28], $3.14(2))$, the map (6.6.2) is naturally identified with the isomorphism

$$
\operatorname{Hom}\left(\Omega_{X_{\mathcal{B}} / \mathcal{B}}^{1}, I\right) \simeq \operatorname{Hom}\left(\Omega_{X / \mathcal{A}}^{1}, I\right)
$$

Combining (6.6) with the fact that Pic is an algebraic space, we conclude that the functor in condition $\left(\mathrm{S} 1^{\log }\right)$ is an equivalence.

To see that $\left(\mathrm{S} 2^{\log }\right.$ ) holds, let $\mathcal{A} \rightarrow \mathcal{A}_{0}$ be a surjective morphism of noetherian log rings as in (B.6) and let $I$ be an $A_{0}$-module of finite type. Suppose given a special polarized log K3 surface $(X, \lambda)$ over $\mathcal{A}$. Denote by $\left(X_{\mathcal{A}[I]}, \lambda_{\mathcal{A}[I]}\right)$ the polarized log K3 surface over $\mathcal{A}$ obtained by pullback via the retraction $\mathcal{A} \rightarrow \mathcal{A}[I]$. Since $H^{1}\left(X, I \otimes \mathcal{O}_{X}\right)=0$, the exponential sequence (4.12.1) shows that the map

$$
H^{1}\left(X_{\mathcal{A}[I]}, \mathcal{M}_{X_{\mathcal{A}[I]}^{\mathrm{gp}}}\right) \longrightarrow H^{1}\left(X, \mathcal{M}_{X}^{\mathrm{gp}}\right)
$$

is injective. From this we deduce that any automorphism of $X_{\mathcal{A}[I]}$, viewed as a log smooth deformation of $X$ to $\mathcal{A}[I]$, is compatible with the polarizations. Hence by ([28], $3.14(2)$ ) there is a natural isomorphism

$$
\operatorname{Aut}_{X}\left(\mathcal{A}_{0}[I]\right) \simeq \operatorname{Hom}\left(\Omega_{X / \mathcal{A}}^{1}, I \otimes \mathcal{O}_{X}\right)
$$

in particular, $\operatorname{Aut}_{X}\left(\mathcal{A}_{0}[I]\right)$ is of finite type.

To describe the tangent space $\mathrm{T}_{\left(\mathbb{M}_{2 k}, \mathcal{M}_{\mathbb{M}_{2 k}}\right) /\left(\operatorname{Spec}(\mathbb{Q}), \mathbb{Q}^{*}\right), X}(I)$ (which we henceforth abbreviate $\mathrm{T}_{X}(I)$ ), note that the injectivity of (6.6.3) implies that $\mathrm{T}_{X}(I)$ is in bijection with the set of $\log$ smooth liftings of $X$ to $\mathcal{A}[I]$ to which $\lambda$ lifts. By ([28], 3.14 (3)), we thus have a natural inclusion

$$
\mathrm{T}_{X}(I) \subset \operatorname{Ext}^{1}\left(\Omega_{X / \mathcal{A}}^{1}, I \otimes \mathcal{O}_{X}\right) .
$$

Since the $A_{0}$-module $\operatorname{Ext}^{1}\left(\Omega_{X / \mathcal{A}}^{1}, I \otimes \mathcal{O}_{X}\right)$ is finitely generated and $A_{0}$ is noetherian, it follows that $\mathrm{T}_{X}(I)$ is of finite type.

6.7. $\mathbb{M}_{2 k}$ is formally log smooth.

Let $\mathcal{A}^{\prime} \rightarrow \mathcal{A} \rightarrow \mathcal{A}_{0}$ be a diagram of artinian local log rings as in (B.6), and let $I:=$ $\operatorname{Ker}\left(A^{\prime} \rightarrow A\right)$. We claim that for any special polarized log $\mathrm{K} 3$ surface $(X, \lambda)$ over $\mathcal{A}$, there exists étale locally on $\operatorname{Spec}(A)$ a lifting of $(X, \lambda)$ to $\mathcal{A}^{\prime}$.

To prove this, we can without loss of generality assume that $\lambda$ is induced by a line bundle $\mathcal{L}$ on $X$.

Lemma 6.8. Let $X^{\prime} / \mathcal{A}^{\prime}$ be a log smooth lifting of $X$ to $\mathcal{A}^{\prime}$. Then $\lambda$ lifts to $X^{\prime}$ if and only if $\lambda$ lifts étale locally on $\operatorname{Spec}(A)$ to $X^{\prime}$. Moreover, $\lambda$ lifts to $X^{\prime}$ if and only if $\mathcal{L}$ lifts to a line bundle on $X^{\prime}$.

Proof. First observe that the condition that $\mathcal{L}$ lifts to $X^{\prime}$ is étale local on $\operatorname{Spec}(A)$. Indeed consider the stack $F$ over the étale site of $\operatorname{Spec}(A)$ which to any étale $U \rightarrow \operatorname{Spec}(A)$ associates the groupoid of liftings of $\left.\underset{\mathcal{L}}{\mathcal{L}}\right|_{X_{U}}$ to $X_{U}^{\prime}$ (where $X_{U}=\stackrel{\circ}{X} \times_{\operatorname{Spec}(A)} U$ and $X_{U}^{\prime}$ denotes the unique lifting of $X_{U}$ to an étale $X^{\prime}$-scheme). From the exponential sequence

$$
0 \longrightarrow I \otimes \mathcal{O}_{X} \longrightarrow \mathcal{O}_{X^{\prime}}^{*} \longrightarrow \mathcal{O}_{X}^{*} \longrightarrow 0
$$


and the fact that $H^{1}\left(X, I \otimes \mathcal{O}_{X}\right)=0$ (this follows from (5.5)), we see that any two liftings of $\mathcal{L}$ to $X^{\prime}$ are isomorphic. Moreover, for any lifting $\mathcal{L}^{\prime}$ of $\mathcal{L}$ to $X^{\prime}$, the group of infinitesimal automorphisms of $\mathcal{L}^{\prime}$ is canonically identified with

$$
\operatorname{Hom}(\mathcal{L}, I \otimes \mathcal{L}) \simeq H^{0}\left(X, I \otimes \mathcal{O}_{X}\right)
$$

Thus if there exists étale locally on $\operatorname{Spec}(A)$ a lifting of $\mathcal{L}$, then $F$ is a gerbe under the coherent sheaf $f_{*}\left(I \otimes \mathcal{O}_{X}\right)$ on $\operatorname{Spec}(A)$ (where $f: \stackrel{\circ}{X} \rightarrow \operatorname{Spec}(A)$ denotes the structure morphism). Since $H^{2}\left(\operatorname{Spec}(A), f_{*}\left(I \otimes \mathcal{O}_{X}\right)\right)=0$, it follows from ([21], IV.3.4) that $F$ is trivial.

Thus to prove the lemma it suffices to show that if $\lambda$ lifts étale locally on $\operatorname{Spec}(A)$ to $X^{\prime}$, then $\mathcal{L}$ also lifts étale locally to $X^{\prime}$. To show this, we can replace $\operatorname{Spec}(A)$ by an étale cover, and hence may assume that we have a class $\lambda^{\prime} \in H^{1}\left(X^{\prime}, \mathcal{M}_{X^{\prime}}^{\mathrm{gp}}\right)$ inducing $\lambda$. Since we have a lifting of $\lambda$, the image of $\lambda$ under the map

$$
H^{1}\left(X, \mathcal{M}_{X}^{\text {gp }}\right) \longrightarrow H^{2}\left(X, I \otimes \mathcal{O}_{X}\right)
$$

obtained from the exponential sequence

$$
0 \longrightarrow I \otimes \mathcal{O}_{X} \longrightarrow \mathcal{M}_{X^{\prime}}^{\mathrm{gp}} \longrightarrow \mathcal{M}_{X}^{\mathrm{gp}} \longrightarrow 0
$$

is zero. On the other hand, there is a natural morphism of exact sequences

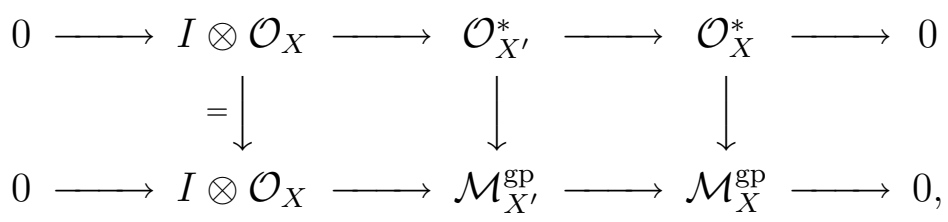

from which we see that the image of the class of $\mathcal{L}$ under the boundary map

$$
H^{1}\left(X, \mathcal{O}_{X}^{*}\right) \longrightarrow H^{2}\left(X, I \otimes \mathcal{O}_{X}\right)
$$

is zero. From this it follows that $\mathcal{L}$ also lifts to $X^{\prime}$.

Now by $([29], 2.5)$, there exists a log smooth lifting $X^{\prime} / \mathcal{A}^{\prime}$ of $X$. Let $o \in H^{2}\left(X, I \otimes \mathcal{O}_{X}\right)$ be the image of $\mathcal{L}$ under the resulting map (6.8.1), and let

$$
d \log : \mathcal{O}_{X}^{*} \longrightarrow \Omega_{X / \mathcal{A}}^{1}
$$

be the map which sends $u$ to $u^{-1} d u$. If $X^{\prime \prime}$ is a second $\log$ smooth lifting of $X$ to $\mathcal{A}^{\prime}$ with associated obstruction $o^{\prime} \in H^{2}\left(X, I \otimes \mathcal{O}_{X}\right)$ to lifting $\mathcal{L}$, then one shows as in the classical case (see for example $([38], 1.14)$ ) that

$$
o^{\prime}=<\left[X^{\prime \prime}\right]-\left[X^{\prime}\right], d \log (\mathcal{L})>+o,
$$

where $\left[X^{\prime \prime}\right]-\left[X^{\prime}\right] \in \operatorname{Ext}^{1}\left(\Omega_{X / \mathcal{A}}^{1}, I \otimes \mathcal{O}_{X}\right)$ denotes the class obtained from the torsorial action of $\operatorname{Ext}^{1}\left(\Omega_{X / \mathcal{A}}^{1}, I \otimes \mathcal{O}_{X}\right)$ on the isomorphism classes of log smooth deformations of $X$ ([28], $3.14(2))$, and

$$
<\cdot, \cdot>: \operatorname{Ext}^{1}\left(\Omega_{X / \mathcal{A}}^{1}, I \otimes \mathcal{O}_{X}\right) \otimes H^{1}\left(X, \Omega_{X / \mathcal{A}}^{1}\right) \longrightarrow H^{2}\left(X, I \otimes \mathcal{O}_{X}\right)
$$

is the natural pairing. The following lemma therefore shows that there exists a lifting of $(X, \lambda)$ to $\mathcal{A}^{\prime}$. 
Lemma 6.9. The map

$$
<\cdot d \log (\mathcal{L})>: \operatorname{Ext}^{1}\left(\Omega_{X / \mathcal{A}}^{1}, I \otimes \mathcal{O}_{X}\right) \longrightarrow H^{2}\left(X, I \otimes \mathcal{O}_{X}\right)
$$

is surjective.

Proof. Let $\kappa$ be the residue field of $A$. Since we are working in characteristic 0 , we reduce in a standard way to the case when $\kappa=\mathbb{C}$. Moreover, since $I$ is a $\kappa$-vector space, we can without loss of generality assume that $I=\kappa$, and so we need to show that the map

$$
<\cdot d \log (\mathcal{L})>: \operatorname{Ext}^{1}\left(\Omega_{X_{\kappa}}^{1}, \mathcal{O}_{X_{\kappa}}\right) \longrightarrow H^{2}\left(X_{\kappa}, \mathcal{O}_{X_{\kappa}}\right)
$$

is surjective, where $X_{\kappa}$ denotes the reduction of $X$ to $\kappa$ and $\Omega_{X_{\kappa}}^{1}$ denotes the log differentials of $X_{\kappa}$ over $\operatorname{Spec}(\kappa)$ with the $\log$ structure induced by $\mathcal{M}_{\mathcal{A}}$. Since $H^{2}\left(X_{\kappa}, \mathcal{O}_{X_{\kappa}}\right)$ is a 1-dimensional $\kappa$-space by $(5.5)$, it suffices to find a class in $\operatorname{Ext}^{1}\left(\Omega_{X_{\kappa}}^{1}, \mathcal{O}_{X_{\kappa}}\right)$ whose pairing with $d \log (\mathcal{L})$ is non-zero.

Let $\Omega_{X_{\kappa}}^{1}$ denote the usual differentials of the space $\stackrel{\circ}{X}_{\kappa}$ over $\kappa$. By $([46], 4.4)$, the flat deformations of $\stackrel{\circ}{X}_{\kappa}$ to the dual numbers $\kappa[\epsilon]$ are classified by the space $\operatorname{Ext}^{1}\left(\Omega_{X_{\kappa}}^{1}, \mathcal{O}_{X_{\kappa}}\right)$. The natural map $\Omega_{X_{\kappa}}^{1} \rightarrow \Omega_{X_{\kappa}}^{1}$ induces a map

$$
\operatorname{Ext}^{1}\left(\Omega_{X_{\kappa}}^{1}, \mathcal{O}_{X}\right) \longrightarrow \operatorname{Ext}^{1}\left(\Omega_{X_{\kappa}}^{1}, \mathcal{O}_{X}\right)
$$

which has the following modular interpretation. Let $\mathcal{M}_{\kappa[\epsilon]}$ be the pullback of $\mathcal{M}_{\kappa}$ to $\kappa[\epsilon]$ via the natural retraction $\kappa \rightarrow \kappa[\epsilon]$. Then the left hand side of (6.9.3) classifies log smooth deformations of $X_{\kappa}$ to $\left(\operatorname{Spec}(\kappa[\epsilon]), \mathcal{M}_{\kappa[\epsilon]}\right)$ and the map (6.9.3) is obtained by sending a $\log$ smooth deformation $X^{\prime} /\left(\operatorname{Spec}(\kappa[\epsilon]), \mathcal{M}_{\kappa[\epsilon]}\right)$ to the underlying space $\stackrel{\circ}{X^{\prime}}$. By $(2.5)$ and $(2.8)$, two $\log$ smooth deformations $X_{1}$ and $X_{2}$ of $X_{\kappa}$ to $\left(\operatorname{Spec}(\kappa[\epsilon]), \mathcal{M}_{\kappa[\epsilon]}\right)$ induce the same element in $\operatorname{Ext}^{1}\left(\Omega_{X_{\kappa}}^{1}, \mathcal{O}_{X}\right)$ if and only if there exists an automorphism $\sigma$ of $\mathcal{M}_{\kappa[\epsilon]}$ which induces the identity on $\mathcal{M}_{\kappa}$ such that $X_{2}$ is isomorphic to the deformation $X_{1}^{\sigma}$ of $X_{\kappa}$ whose log scheme is equal to $X_{1}$ but whose structure morphism is the composite

$$
X_{1} \longrightarrow\left(\operatorname{Spec}(\kappa[\epsilon]), \mathcal{M}_{\kappa[\epsilon]}\right) \stackrel{(i d, \sigma)}{\longrightarrow}\left(\operatorname{Spec}(\kappa[\epsilon]), \mathcal{M}_{\kappa[\epsilon]}\right) .
$$

In other words, the kernel of (6.9.3) is naturally identified with the group of infinitesimal automorphisms of $\mathcal{M}_{\kappa[\epsilon]}$, which is a 1-dimensional subspace isomorphic to

$$
\operatorname{Hom}\left(\overline{\mathcal{M}}_{\kappa}, \kappa\right) \simeq \operatorname{Hom}(\mathbb{N}, \kappa) \simeq \kappa .
$$

From this and (5.5), we conclude that the image of (6.9.3) is 19-dimensional. On the other hand, it follows from (proofs of $([16], 4.3)$ and $([20], 5.5))$ that the map

$$
<\cdot d \log (\mathcal{L})>: \operatorname{Ext}^{1}\left(\Omega_{X_{\kappa}}^{1}, \mathcal{O}_{X}\right) \longrightarrow H^{2}\left(X_{\kappa}, \mathcal{O}_{X_{\kappa}}\right)
$$

is surjective with 19-dimensional kernel. Thus to prove the lemma, it suffices to show that there is an element in the kernel of (6.9.4) which is not in the image of (6.9.3). This follows from $([16], 4.3)$ and $([20], 5.5)$, which imply that there exists a smoothing of the pair $\left(\stackrel{\circ}{X}_{\kappa}, \mathcal{L}\right)$. Indeed the underlying space of any $\log$ smooth deformation of $X_{\kappa}$ to $\left(\operatorname{Spec}(\kappa[\epsilon]), \mathcal{M}_{\kappa[\epsilon]}\right)$ is étale locally of the form

$$
\kappa[\epsilon][x, y, z] /(x y \text { or } x y z)
$$


and hence is not isomorphic to the reduction $\bmod t^{2}$ of a smoothing of $\stackrel{\circ}{X}_{\kappa}$ to $\kappa[[t]]$.

\subsection{The additional conditions (B.11).}

Let $\mathcal{A} \rightarrow \mathcal{A}_{0}$ and $I$ be as in (B.6). A polarization $\lambda$ on a special log K3 surface $X / \mathcal{A}$ defines in a natural way a class $d \log (\lambda) \in H^{1}\left(X, \Omega_{X / \mathcal{A}}^{1}\right)$. Indeed, the map

$$
d \log : \mathcal{M}_{X}^{\mathrm{gp}} \longrightarrow \Omega_{X / \mathcal{A}}^{1}
$$

obtained from the universal log derivation ([27], 5.1) induces a map of presheaves

$$
\left(U / \operatorname{Spec}(\mathcal{A}) \longmapsto H^{1}\left(X_{U}, \mathcal{M}_{X_{U}}^{\mathrm{gp}}\right)\right) \longrightarrow\left(U / \operatorname{Spec}(A) \longmapsto H^{1}\left(X_{U}, \Omega_{X_{U} / U}^{1}\right)\right),
$$

where $U / \operatorname{Spec}(\mathcal{A})$ is a strict étale morphism and $X_{U}$ denotes the base change of $X$. If $f: \stackrel{\circ}{X} \rightarrow \operatorname{Spec}(A)$ denotes the structure morphism we therefore obtain a map of sheaves on the étale site of $\operatorname{Spec}(A)$

and hence a map

$$
\underline{\mathrm{Pic}} \longrightarrow R^{1} f_{*} \Omega_{X / \mathcal{A}}^{1}
$$

$$
d \log : \underline{\operatorname{Pic}}(\mathcal{A}) \longrightarrow H^{1}\left(X, \Omega_{X / \mathcal{A}}^{1}\right) .
$$

It follows from the proof of (6.8) that $T_{X}(I)$ is isomorphic to

$$
\operatorname{Ker}\left(<\cdot, d \log (\lambda)>: \operatorname{Ext}^{1}\left(\Omega_{X / \mathcal{A}}^{1}, I \otimes \mathcal{O}_{X}\right) \longrightarrow H^{2}\left(X, I \otimes \mathcal{O}_{X}\right)\right)
$$

From this, standard properties of cohomology, and the description of the module of infinitesimal automorphisms given in (6.5) we deduce that the additional conditions (B.11) hold.

\subsection{Compatibility with completion.}

Let $\hat{\mathcal{A}}$ denote a complete noetherian local ring $\hat{A}$ with residue field $\kappa$ together with a $\log$ structure $\mathcal{M}_{\hat{A}}$. Denote by $\hat{\mathcal{A}}_{n}$ the reduction modulo $\mathfrak{m}^{n+1}$. We show that the natural functor (see (B.3) for the notation)

$$
\left(\mathbb{M}_{2 k}, \mathcal{M}_{\mathbb{M}_{2 k}}\right)_{\hat{\mathcal{A}}} \longrightarrow \lim \left(\mathbb{M}_{2 k}, \mathcal{M}_{\mathbb{M}_{2 k}}\right)_{\hat{\mathcal{A}}_{n}}
$$

is an equivalence of categories. For this we may by descent theory assume that $\kappa$ is separably closed.

To see that (6.11.1) is fully faithful, suppose given two polarized log K3 surfaces $\left(X_{1}, \lambda^{1}\right)$ and $\left(X_{2}, \lambda^{2}\right)$ over $\hat{\mathcal{A}}$, and a compatible family $\sigma_{n}$ of isomorphisms between the reductions. By ([14], III.5.4.1) there exists a unique isomorphism $\stackrel{\circ}{X}_{1} \simeq \stackrel{\circ}{X}_{2}$ inducing the isomorphisms $\stackrel{\circ}{\sigma}_{n}: \stackrel{\circ}{X}_{1, n} \simeq \stackrel{\circ}{X}_{2, n}$. By (2.5) and (2.9) this isomorphism can in fact be extended to an isomorphism of $\log$ algebraic spaces inducing the $\sigma_{n}$. This proves fullness of the functor. As for faithfulness suppose $\sigma$ is an automorphism of a polarized $\log \mathrm{K} 3$ surface $(X, \lambda)$. Then the corresponding automorphism of $\stackrel{\circ}{X}$ must be the identity since its graph $\Gamma \subset \stackrel{\circ}{X} \times \stackrel{\circ}{X}$ is a closed subspace, and hence determined by its reductions by ([14], III.5.1.8). The fact that $\sigma$ must also be the identity on the $\log$ structure of $X$ then follows from another application of (2.9).

To prove the essential surjectivity of (6.11.1), we use a modification of the argument given in ([1], proof of 2.2). Suppose given a compatible family of polarized, stable special log K3 
surfaces $\left(X_{n}, \lambda_{n}\right)$ which we wish to algebraize. Fix a chart $\mathbb{N} \rightarrow \mathcal{M}_{\hat{A}}$ (such a chart exists étale locally). Then the functor over the category of Artinian local $\kappa[[t]]$-algebras with residue field $\kappa$ which to any such algebra $B$ associates the isomorphism classes of liftings of $\left(X_{0}, \lambda_{0}\right)$ to $B$ with $\log$ structure induced by $t$, is pro-representable and formally smooth by Schlessinger's criteria ([43], 2.11). Say the algebra

$$
\kappa[[t]]\left[\left[q_{1}, \ldots, q_{h}\right]\right]
$$

pro-represents this functor. Then the family $\left(X_{n}, \lambda_{n}\right)$ is obtained from a family over the reductions of this ring, and so we may assume that $\hat{A}$ is in fact equal to this ring.

Lemma 6.12. Let $X /\left(\operatorname{Spec}(\kappa), \mathcal{M}_{\kappa}\right)$ be a special log $K 3$ surface, where $\kappa$ is an algebraically closed field, and suppose $\lambda$ is a polarization on $X$. Let $\mathcal{L}$ be a representative line bundle for $\lambda$ for which $\mathcal{L}^{N}$ defines a map $\bar{f}: \stackrel{\circ}{X} \rightarrow \bar{X} \subset \mathbb{P}_{\kappa}^{r}$ contracting only finitely many curves. Then for $N$ sufficiently big,

(i) the natural map $\mathcal{O}_{\bar{X}} \rightarrow R \bar{f}_{*} \mathcal{O}_{X}$ is an isomorphism.

(ii) the group $H^{1}\left(X, \mathcal{L}^{N}\right)$ is zero.

Proof. By (5.17), we may assume that $\bar{f}$ has connected fibers. By a standard application of ([14], III.4.2), to prove (i) it suffices to show that if $F$ denotes the fiber of $\bar{f}$ at a point $x \in \bar{X}$, then

$$
H^{0}\left(F, \mathcal{O}_{F}\right)=\kappa(x), \quad H^{i}\left(F, \mathcal{O}_{F}\right)=0, \quad i>0 .
$$

This follows from the classification of the fibers given in (5.16). Indeed the case of a negative definition configuration of $(\S)$-curves follows from $([6])$, and in the other cases one can show the result by a standard calculation (left to the reader).

To see (ii), let $\mathcal{O}_{\bar{X}}(1)$ be the tautological very ample sheaf on $\bar{X}$. By ([24], III.5.3), there exists an integer $M$ such that $\mathrm{H}^{1}\left(\bar{X}, \mathcal{O}_{\bar{X}}(1)^{\otimes M}\right)=0$. Hence it suffices to show that the natural map

$$
\mathcal{O}_{\bar{X}}(1) \longrightarrow R \bar{f}_{*} \mathcal{L}^{N} \simeq R \bar{f}_{*} \bar{f}^{*} \mathcal{O}_{\bar{X}}(1)
$$

is a quasi-isomorphism. Since $\mathcal{O}_{\bar{X}}(1)$ is locally free this follows from (i).

By the lemma we can choose a representative line bundle $\mathcal{L}_{0}$ for $\lambda_{0}$ and an integer $N$ such that $\mathcal{L}_{0}^{N}$ is generated by global sections, the induced map $\stackrel{\circ}{X}_{0} \rightarrow \bar{X}_{0} \subset \mathbb{P}_{\kappa}^{r}$ contracts only finitely many curves and satisfies $(6.12(\mathrm{i}))$, and $H^{1}\left(X_{0}, \mathcal{L}_{0}^{N}\right)=0$. Moreover, by (6.8), we can then extend $\mathcal{L}_{0}$ to a family $\left\{\mathcal{L}_{n}\right\}$ of invertible sheaves on the $X_{n}$ inducing $\left\{\lambda_{n}\right\}$.

Lemma 6.13. Let $f_{n}: X_{n} \rightarrow \operatorname{Spec}\left(\mathcal{A}_{n}\right)$ denote the structure morphism.

(i) For each $n$, the module $H^{0}\left(X_{n}, \mathcal{L}_{n}^{N}\right)$ is flat over $A_{n}$ and

$$
H^{0}\left(X_{n}, \mathcal{L}_{n}^{N}\right) \otimes_{A_{n}} A_{n-1}=H^{0}\left(X_{n-1}, \mathcal{L}_{n-1}^{N}\right) .
$$

(ii) If $\bar{X}_{n}$ denotes the scheme-theoretic image of the natural map

$$
\bar{f}_{n}: \stackrel{\circ}{X}_{n} \longrightarrow \operatorname{Proj}\left(\operatorname{Sym} \bullet H^{0}\left(X_{n}, \mathcal{L}_{n}^{N}\right)\right),
$$

then $\bar{f}_{n *} \mathcal{O}_{X_{n}}=\mathcal{O}_{\bar{X}_{n}}, \bar{X}_{n}$ is flat over $A_{n}$, and $\bar{X}_{n} \otimes_{A_{n}} A_{n-1}=\bar{X}_{n-1}$. 
Proof. We proceed by induction on $n$. The case $n=0$ follows from (6.12), so we suppose the result holds for $n-1$ and prove it for $n$.

Since $\stackrel{\circ}{X}_{n} / A_{n}$ is flat, there is a natural exact sequence

$$
0 \longrightarrow \mathfrak{m}^{n} / \mathfrak{m}^{n+1} \otimes \mathcal{L}_{0}^{N} \longrightarrow \mathcal{L}_{n}^{N} \longrightarrow \mathcal{L}_{n-1}^{N} \longrightarrow 0,
$$

and since $H^{1}\left(X_{0}, \mathcal{L}_{0}^{N}\right)=0$ it follows that the map

$$
H^{0}\left(X_{n}, \mathcal{L}_{n}^{N}\right) \longrightarrow H^{0}\left(X_{n-1}, \mathcal{L}_{n-1}^{N}\right)
$$

is surjective. From this it follows that $\mathcal{L}_{n}^{N}$ is generated by global sections, and by the local criterion for flatness ([14], $0_{\mathrm{III}}$.10.2.1) the module $H^{0}\left(X_{n}, \mathcal{L}_{n}^{N}\right)$ is flat over $A_{n}$. Moreover, there is a natural morphism of complexes

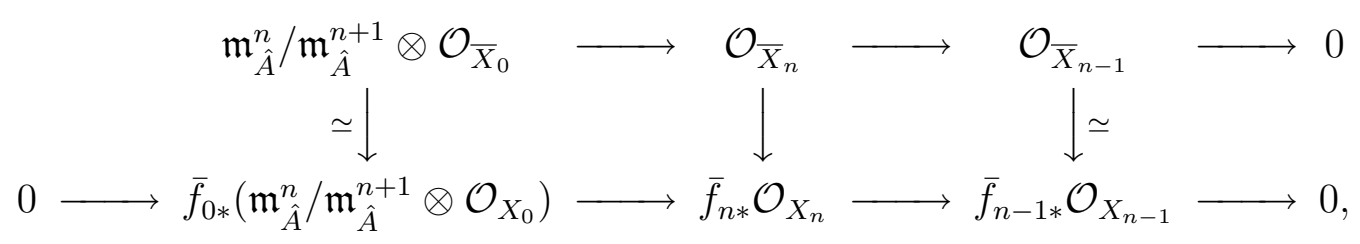

where the bottom row is exact since $R^{1} \bar{f}_{0 *} \mathcal{O}_{X_{0}}=0$ and $(6.12$ (i)) holds. From this it follows that the sequence

$$
0 \longrightarrow \mathfrak{m}^{n} / \mathfrak{m}^{n+1} \otimes \mathcal{O}_{\bar{X}_{0}} \longrightarrow \mathcal{O}_{\bar{X}_{n}} \longrightarrow \mathcal{O}_{\bar{X}_{n-1}} \longrightarrow 0
$$

is exact, and hence by the local criterion for flatness the scheme $\bar{X}_{n}$ is flat over $\hat{A} / \mathfrak{m}_{\hat{A}}^{n+1}$. Moreover, the sequence (6.13.1) implies that $X_{n} \otimes_{A_{n}} A_{n-1}=X_{n-1}$.

6.14. Choosing a compatible set of bases for the $H^{0}\left(X_{n}, \mathcal{L}_{n}^{N}\right)$, we obtain a compatible family of flat subschemes $\bar{X}_{n} \subset \mathbb{P}_{A_{n}}^{r}$ (for some $r$ ). By the Grothendieck existence theorem ([14], III.5.1.4) this family is induced by a flat projective scheme $\bar{X} / \hat{A}$. Note also that $\bar{X}$ is integral. Indeed since $\bar{X}$ is proper over $\hat{A}$, the local ring of every point is a localization of the local ring at a point in the closed fiber, and the integrality of $\bar{X}$ at points in the closed fiber can be verified after passing to the formal completion $\bar{X}$ of $\bar{X}$ along $\mathfrak{m}_{\hat{A}}$. Now if $g: X \rightarrow \bar{X}$ denotes the map of formal schemes, obtained from the $X_{n}$ then there is an inclusion $\mathcal{O}_{\bar{x}} \hookrightarrow g_{*} \mathcal{O}_{x}$, and hence it suffices to verify that $\mathcal{X}$ is integral. But for any point $p \in X_{0}$, there exists an étale neighborhood $U_{0}$ of $p$ and an étale morphism

$$
U_{0} \longrightarrow \operatorname{Spec}(\kappa[x, y, z] /(x y \text { or } x y z))
$$

inducing the log structure $\mathcal{M}_{X_{0}}$ restricted to $U_{0}$. Since each $X_{n}$ is $\log \operatorname{smooth}$ over $\operatorname{Spec}\left(\mathcal{A}_{n}\right)$, we can extend the map (6.14.1) to a compatible family of étale maps

$$
U_{n} \longrightarrow \operatorname{Spec}\left(\hat{A}_{n}[x, y, z] /(x y-t \text { or } x y z-t)\right),
$$

and from this it follows that $\mathcal{X}$ is integral.

6.15. Next the issue of finding a proper algebraic space $\stackrel{\circ}{X} / \bar{X}$ inducing $\stackrel{\circ}{X}_{n} / \bar{X}_{n}$ is étale local on $\bar{X}$. To see this, suppose we can find an étale cover $\bar{U} \rightarrow \bar{X}$ and a proper morphism $U \rightarrow \bar{U}$ inducing the family $\left\{U_{n}:=\stackrel{\circ}{X}_{n} \times_{\bar{X}} U\right\}$. Then we claim that we can find $\stackrel{\circ}{X} / \bar{X}$ inducing $\stackrel{\circ}{X}_{n}$. Indeed note that for each point $\bar{p} \in \bar{U}_{0}$ in the exceptional set and lifting $p \in U_{0}$ of $\bar{p}$ we have $\kappa(\bar{p})=\kappa(p)$ since $\kappa$ is algebraically closed. Choose $\bar{\Delta} \subset \bar{X}$ a closed subscheme whose 
reduction $\Delta_{0}$ is set-theoretically equal to the exceptional set of $\stackrel{\circ}{X}_{0} / \bar{X}_{0}$. Since $\bar{U} / \bar{X}$ is étale and $\kappa(p)=\kappa(\bar{p})$ for each point in the exceptional set, we can lift $\bar{\Delta}$ to a closed subscheme of $\bar{U}$ which we denote by the same symbol. Thus if $\overline{\mathcal{U}}$ and $\bar{X}$ denote the formal completions along $\bar{\Delta}$, we have an isomorphism $\overline{\mathcal{U}} \simeq \bar{X}$. If $\mathcal{U}$ denotes the formal completion of $U$ along the inverse image of $\bar{\Delta}$, then $\mathcal{U} \rightarrow \overline{\mathcal{U}} \simeq \bar{X}$ is a formal modification in the sense of ([3], 1.7); hence by $([3], 3.2)$ there exists a unique proper morphism $\stackrel{\circ}{X} \rightarrow \bar{X}$ inducing $U / \bar{U}$ and hence also $\stackrel{\circ}{X}_{n}$. Thus to construct the space $\stackrel{\circ}{X}$ we may replace $\bar{X}$ by any étale neighborhood of the exceptional locus.

Now choose an étale morphism $\bar{U} \rightarrow \bar{X}$ such that $\pi_{0}: U_{0} \rightarrow \bar{U}_{0}$ is relatively projective and such that $\bar{U}=\operatorname{Spec}(B)$ is affine. Denote by $\mathcal{J}_{0}$ a relatively ample line bundle on $U_{0}$. Let $B^{\dagger}=\lim _{\longleftarrow} B / \mathfrak{m}_{\hat{A}}^{n} B$ and let $\mathcal{U} \rightarrow \overline{\mathcal{U}}:=\operatorname{Spf}\left(B^{\dagger}\right)$ be the formal scheme obtained from the $U_{n}$. Since $\overleftarrow{U}_{0}$ is affine and the fibers of $U_{0} \rightarrow \bar{U}_{0}$ have dimension $\leq 1$, the spectral sequence

$$
E_{2}^{p, q}=H^{q}\left(\bar{U}_{0}, R^{p} \pi_{0 *} \mathcal{O}_{U_{0}}\right) \Longrightarrow H^{p+q}\left(U_{0}, \mathcal{O}_{U_{0}}\right)
$$

shows that $H^{2}\left(U_{0}, \mathcal{O}_{U_{0}}\right)=0$. As discussed in the proof of (6.8), the obstruction to lifting $\mathcal{J}_{0}$ to the $U_{n}$ lies in $H^{2}\left(U_{0}, \mathcal{O}_{U_{0}}\right)$, and hence there exists a formal line bundle $\widehat{\mathcal{J}}$ on $\mathcal{U}$ which is relatively ample over $\overline{\mathcal{U}}([14], 4.7 .1)$. It follows from the Grothendieck existence theorem ([14], III.5.1.4) that $\mathcal{U}$ is induced by a proper scheme $\widehat{U} / B^{\dagger}$. The map $\widehat{U} \rightarrow \operatorname{Spec}\left(B^{\dagger}\right)$ is an isomorphism outside of some closed subscheme $\Gamma \subset \operatorname{Spec}\left(B^{\dagger}\right)$ whose reduction is settheoretically contained in the exceptional locus. It follows that $\Gamma$ is finite over $\hat{A}$ and hence is induced by an isomorphic closed subscheme (which we denote by the same letter) $\Gamma \subset$ $\operatorname{Spec}(B)$.

Since $\widehat{U} \rightarrow \operatorname{Spec}\left(B^{\dagger}\right)$ is projective and $\widehat{U}$ has no embedded components lying over $\Gamma$ (this can be checked after reduction), $\widehat{U}$ is the blow-up of some sheaf of ideals $\bar{J} \subset B^{\dagger}$ ([14], III.2.3.7). Set $I=($ ideal of $\Gamma) \cap \mathfrak{m} B \subset B$.

Lemma 6.16. Let $B_{I}^{\dagger}$ denote the I-adic completion of $B$. Then the natural map $B_{I}^{\dagger} \rightarrow B^{\dagger}$ is an isomorphism.

Proof. Let $K$ denote the ideal of $\Gamma$, and consider the exact sequences

$$
0 \longrightarrow \mathfrak{m}^{n} B / I^{n} \longrightarrow B / I^{n} \longrightarrow B / \mathfrak{m}^{n} B \longrightarrow 0 .
$$

Since $\hat{A}$ is noetherian and $\mathfrak{m}^{n} B / I^{n}$ is a $B / K^{n}$-module, $\mathfrak{m}^{n} B / I^{n}$ is a finitely generated $\hat{A}$ module, and hence $\cap_{r} \mathfrak{m}^{r}\left(\mathfrak{m}^{n} B / I^{n}\right)=0$. Thus $\lim _{n} \mathfrak{m}^{n} B / I^{n}=0$ and $B_{I}^{\dagger} \rightarrow B^{\dagger}$ is injective.

For the surjectivity, suppose $\left\{f_{n}\right\}$ is a compatible sequence of elements in $B / \mathfrak{m}^{n}$, and for each $n$ choose a lifting $g_{n} \in B / I^{n}$ of $f_{n}$. For each $r \geq 0$, the image of $g_{n+r+1}-g_{n+r}$ in $B / I^{n}$ is contained in $\mathfrak{m}^{r}\left(\mathfrak{m}^{n} B / I^{n}\right)$. Since $\mathfrak{m}^{n} B / I^{n}$ is finitely generated over $\hat{A}$ it is $\mathfrak{m}$-adically complete, and so we can define elements

$$
r_{n}:=\sum_{r \geq 0} \operatorname{Im}\left(g_{n+r+1}-g_{n+r}\right) \in \mathfrak{m}^{n} B / I^{n} .
$$

Letting $g_{n}^{*}:=g_{n}+r_{n}$ we obtain a compatible family $\left\{g_{n}^{*}\right\}$ inducing $\left\{f_{n}\right\}$. 
Let $\widetilde{B}$ be the henselization of the pair $(B, I)([15], 0.1)$. Then by ([15], page 574) there exists an ideal $\widetilde{J} \subset \widetilde{B}$ inducing $\bar{J}$. Let $\widetilde{U}$ be the blow-up of $\widetilde{J}$ so that $\widetilde{U} \otimes_{\widetilde{B}} \bar{B} \simeq \widehat{U}$. We can now descend $\widetilde{U}$ to some étale neighborhood of $V(I)$ in $\operatorname{Spec}(B)$. Since the problem of finding $\stackrel{\circ}{X} \rightarrow \bar{X}$ inducing $\stackrel{\circ}{X}_{n}$ was shown to be étale local on $\bar{X}, \widetilde{U}$ yields such an $\stackrel{\circ}{X}$.

The space $\stackrel{\circ}{X}$ is étale locally of the form

$$
\hat{A}[x, y, z] /(x y-t \text { or } x y z-t) \text {. }
$$

Indeed it was shown in (6.14) that for every point $p \in \stackrel{\circ}{X}_{0}$, there is an isomorphism

$$
\widehat{\mathcal{O}}_{X, p} \simeq \hat{A}[[x, y, z]] /(x y-t \text { or } x y z-t) .
$$

That $\stackrel{\circ}{X}$ is étale locally of the form (6.16.1) then follows from the Artin approximation theorem and the properness of $\stackrel{\circ}{X} / \hat{A}([4], 2.6)$.

Now by the representability of the functor IVLS in $(2.5), \stackrel{\circ}{X}$ has a unique structure of a special $\log$ smooth algebraic space $X$ over $\operatorname{Spec}(\hat{\mathcal{A}})$. By $\left([14]\right.$, III.5.1.4) $\Omega_{X / \hat{\mathcal{A}}}^{2} \simeq \mathcal{O}_{X}$, and the

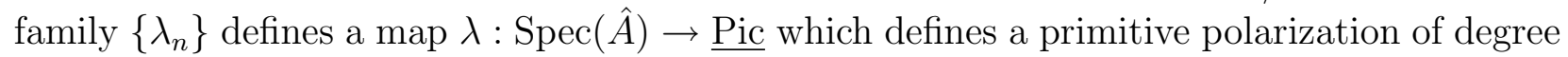
$2 k$ at every point of $\operatorname{Spec}(A)$ by the following proposition:

Proposition 6.17. Let $X \rightarrow S$ be a special log K3 surface, and let $\lambda \in \underline{\operatorname{Pic}}(\stackrel{\circ}{S})$. Then the condition that $\lambda$ defines a primitive polarization of degree $2 k$ is represented by an open subscheme of $\stackrel{\circ}{S}$.

The proof is in several steps (6.18)-(6.21).

Note first that by the argument used in (6.4), we may assume that $\stackrel{\circ}{S}$ is noetherian. Furthermore, by base changing to the irreducible components of $\stackrel{\circ}{S}$ with their reduced structure, we reduce the proof of (6.17) to the case when $\stackrel{\circ}{S}$ is integral. Let $p \in \stackrel{\circ}{S}$ be a point for which the restriction of $\lambda$ to the fiber $X_{p}$ is a polarization of degree $2 k$.

Lemma 6.18. In some étale neighborhood of $p$ there exists a line bundle $\mathcal{L}$ representing $\lambda$ and an integer $N$ such that the following hold:

(i) The natural map $f^{*} f_{*} \mathcal{L}^{N} \rightarrow \mathcal{L}^{N}$ is surjective, where $f: \stackrel{\circ}{X} \rightarrow \stackrel{\circ}{S}$ denotes the structure morphism and $f_{*} \mathcal{L}^{N}$ is locally free.

(ii) The non-trivial fibers of the induced map

$$
\bar{f}: \stackrel{\circ}{X} \longrightarrow \operatorname{Proj}\left(\operatorname{Sym}^{\bullet}\left(f_{*} \mathcal{L}^{N}\right)\right)
$$

consists of curves on $X$, and the scheme theoretic image $\bar{X}$ is flat over $\stackrel{\circ}{S}$.

Proof. First note that it suffices to verify the lemma in the case when $\stackrel{\circ}{S}=\operatorname{Spec}\left(\widehat{\mathcal{O}}_{S, p}\right)$. Indeed suppose we construct a line bundle $\widehat{\mathcal{L}}$ over $\stackrel{\circ}{X} \times{ }_{S} \operatorname{Spec}\left(\widehat{\mathcal{O}}_{S, p}\right)$ satisfying (i) and (ii). Then by the Artin approximation theorem ([4], 2.2), we can after replacing $S$ by an étale neighborhood 
of $p$ find a line bundle $\mathcal{L}$ on $\stackrel{\circ}{X}$ inducing $\widehat{\mathcal{L}}$. Then the support of the cokernel of the natural map $f^{*} f_{*} \mathcal{L}^{N} \rightarrow \mathcal{L}^{N}$ is a closed subset of $S$, and since $f$ is proper its image under $f$ is a closed subset of $S$ which does not contain $p$. Thus after shrinking $S$ we can assume that $f^{*} f_{*} \mathcal{L}^{N} \rightarrow \mathcal{L}^{N}$ is surjective. Moreover, the condition that $f_{*} \mathcal{L}^{N}$ is locally free is an open condition on $S$, and by assumption $f_{*} \mathcal{L}^{N}$ is locally free in a neighborhood of $p$. Hence after shrinking $S$ some more condition (i) holds. Similarly, the condition that the fibers of the resulting map (6.18.1) have dimension $\leq 1$ is an open condition on $\bar{X}$ by the following (6.19), as is the condition that $\bar{X}$ is flat over $\stackrel{\circ}{S}([14]$, IV.11.1.1). Thus since $\bar{X} / \stackrel{\circ}{S}$ is proper we can after shrinking $\stackrel{\circ}{S}$ some more ensure that (ii) holds.

Sub-Lemma 6.19. Let $f: X \rightarrow Y$ be a proper morphism between schemes of finite presentation over a scheme $S / \mathbb{Q}$, and let $r$ be an integer. Then the set of points $y \in Y$ for which $\operatorname{dim}\left(X_{y}\right) \leq r$ is an open set.

Proof. Since $X$ and $Y$ are of finite presentation over $S$, one reduces by a standard limit argument to the case when $S$, and hence also $X$ and $Y$, are of finite type over $\mathbb{Q}$. Moreover, by base changing to the irreducible components of $Y$ with their reduced structure we may assume that $Y$ is integral. Let $\left\{X_{i}\right\}$ be the irreducible components of $X$ with their reduced structure. For each $i$, let $U_{i} \subset Y$ be the set of points $y$ for which $\operatorname{dim}\left(X_{i, y}\right) \leq r$. Then the set of points $y \in Y$ for which $\operatorname{dim}\left(X_{y}\right) \leq r$ is equal to $\cap U_{i}$, and hence it suffices to show that each of the $U_{i}$ are open. Thus we may also assume that $X$ is integral. Let $E$ be the set of points $x \in X$ for which there exists an irreducible component $Z$ of $X_{f(x)}$ containing $x$ such that $\operatorname{dim}(Z) \geq r+1$. By ([24], II.3.22 (d)), the set $E$ is closed. Since $f$ is proper, $f(Z) \subset Y$ is a closed set, and hence its complement is open.

Thus suppose $\stackrel{\circ}{S}=\operatorname{Spec}(\hat{A})$ is the spectrum of a complete noetherian local ring $\hat{A}$ and that $p$ is the closed point. Denote by $X_{n}$ the reduction of $X$ modulo $\mathfrak{m}_{\hat{A}}^{n+1}$. After replacing $\hat{A}$ by an étale cover, we can by the definition of a polarization find a line bundle $\mathcal{L}_{0}$ representing $\lambda$ over $X_{0}$ such that (i) and (ii) hold for the morphism $X_{0} \rightarrow \operatorname{Spec}(\kappa(p))$. Moreover, by (6.12) we may assume that $H^{1}\left(X_{0}, \mathcal{L}_{0}^{N}\right)=0$ and $\bar{f}_{*} \mathcal{O}_{X_{0}}=\mathcal{O}_{\bar{X}_{0}}$, where $\bar{X}_{0}$ denotes the image of $X_{0}$.

In this case, we can by (6.8) find a family $\left\{\mathcal{L}_{n}\right\}$ on $X_{n}$ inducing $\left\{\lambda_{n}\right\}$ such that (i) and (ii) also hold for $X_{n}$. By the Grothendieck existence theorem ([14], III.5.1.4), the family $\left\{\mathcal{L}_{n}\right\}$ is induced by a line bundle $\mathcal{L}$ on $X$ which induces $\lambda$. Now by $\left([14]\right.$, III.4.2), $H^{0}\left(X, \mathcal{L}^{N}\right)=$ $\underset{\lim }{\longleftarrow} H^{0}\left(X_{n}, \mathcal{L}_{n}^{N}\right)$, and hence the module $H^{0}\left(X, \mathcal{L}^{N}\right)$ is flat over $\hat{A}$, and the map $f^{*} f_{*} \mathcal{L}^{N} \rightarrow \mathcal{L}^{N}$ is surjective at every point in the closed fiber of $X$. Since $f$ is proper, it follows that (i) holds over $S$. Similarly, the reduction of $\bar{X}$ modulo $\mathfrak{m}_{\hat{A}}^{n+1}$ is isomorphic to $\bar{X}_{n}$, and hence by (6.19) and the fact that each $X_{n}$ is flat over $\hat{A} / \mathfrak{m}_{\hat{A}}^{n}$ condition (ii) also holds.

Continuing with the proof of (6.17), we may now assume that we have a line bundle $\mathcal{L}$ on $X$ representing $\lambda$ such that $(6.18$ (i)) and $(6.18$ (ii)) hold. In this case, for each geometric point $\bar{s} \rightarrow \stackrel{\circ}{S}$, the number $\lambda^{2}$ is simply the degree in projective space of the fiber $\bar{X}_{\bar{s}}$ divided by $N^{2}$. Hence by ([24], III.9.10), this number is constant in families. Thus the following completes the proof of (6.17): 
Lemma 6.20. The set of points $s \in \stackrel{\circ}{S}$ for which $\lambda_{\bar{s}}$ is primitive is an open and closed subset of $\stackrel{\circ}{S}$.

Proof. It suffices to prove the following two statements:

(6.20.1) The set of points where $\lambda$ is not primitive is an open set.

(6.20.2) The set of points where $\lambda$ is primitive is stable under generization.

To see (6.20.1), suppose that for some point $p \in \stackrel{\circ}{S}$ there exists an element $\lambda^{\prime}$ in the group $\underline{\operatorname{Pic}}(\operatorname{Spec}(\kappa(p)))$ such that $r \lambda^{\prime}=\lambda$ in $H^{1}\left(X_{\bar{p}}, \mathcal{M}_{X_{\bar{p}}}^{\mathrm{gp}}\right)$. We claim that $\lambda^{\prime}$ can be lifted to $\underline{\operatorname{Pic}}\left(\operatorname{Spec}\left(\widehat{\mathcal{O}}_{S, p}\right)\right)$. Since $\underline{\operatorname{Pic}}$ is an algebraic space, it suffices to show that $\lambda^{\prime}$ can be extended to an object $\hat{\lambda}^{\prime}$ in $\lim _{\longleftarrow} \underline{\operatorname{Pic}}\left(\operatorname{Spec}\left(\mathcal{O}_{S_{n}}\right)\right)$, where $\mathcal{O}_{S_{n}}$ denotes the reduction of $\mathcal{O}_{S, p}$ modulo $\mathfrak{m}_{S, p}^{n+1}$, and for this it suffices to show that if $\lambda_{n-1}^{\prime} \in \underline{\operatorname{Pic}}\left(\operatorname{Spec}\left(\mathcal{O}_{S_{n-1}}\right)\right)$ is a lifting of $\lambda^{\prime}$, then there exists a lifting $\lambda_{n}^{\prime} \in \underline{\operatorname{Pic}}\left(\operatorname{Spec}\left(\mathcal{O}_{S_{n}}\right)\right)$ of $\lambda_{n-1}^{\prime}$. This follows from the discussion of the obstruction theory (6.7) which shows that the obstruction to lifting $\lambda_{n-1}^{\prime}$ to $S_{n}$ is a class

$$
o^{\prime} \in H^{2}\left(X_{p}, \mathfrak{m}_{S, p} / \mathfrak{m}_{S, p}^{n+1} \otimes \mathcal{O}_{X_{p}}\right),
$$

and that $r o^{\prime}$ is the obstruction to lifting the reduction of $\lambda$ modulo $\mathfrak{m}_{S, p}^{n}$. Since $\lambda$ lifts and we are in characteristic 0 , it follows that $o^{\prime}=0$.

Note also that by (6.8), we can assume that we have a line bundle $\widehat{\mathcal{L}}$ on $X \times_{S} \operatorname{Spec}\left(\widehat{\mathcal{O}}_{S, p}\right)$ inducing $\hat{\lambda}^{\prime}$. By the Artin approximation theorem ([4], 2.2), we can extend $\widehat{\mathcal{L}}$ to a line bundle $\mathcal{L}$ in some étale neighborhood of $p$. Then $\lambda$ and $\mathcal{L}^{r}$ define two maps $S \rightarrow \underline{\text { Pic which induce }}$ the same map $\operatorname{Spec}\left(\widehat{\mathcal{O}}_{S, p}\right) \rightarrow \underline{\text { Pic. }}$. Hence after replacing $S$ by another étale neighborhood we find that $\lambda$ and $\mathcal{L}^{r}$ define the same map to $\underline{\text { Pic, }}$, and hence the condition that $\lambda$ is not primitive is an open condition.

As for (6.20.2), it suffices to show that if $\hat{A}$ is a complete local integral domain with a $\log$ structure $\mathcal{M}_{\hat{A}}$ and $(X, \lambda)$ is a special polarized $\mathrm{K} 3$ surface over $\left(\operatorname{Spec}(\hat{A}), \mathcal{M}_{\hat{A}}\right)$, then the restriction $\lambda_{\zeta}$ of $\lambda$ to the generic fiber is primitive if the restriction $\lambda_{0}$ of $\lambda$ to the closed fiber is primitive. After possibly replacing $\hat{A}$ by an extension, we can assume that we have a chart $\mathbb{N} \rightarrow \mathcal{M}_{\hat{A}}$ and that $\kappa(p)$ is algebraically closed. Let $s: \kappa[[t]] \rightarrow \hat{A}$ be the map induced by the chart. Consider the functor $D$ on artinian local $\kappa[[t]]$ algebras with residue field $\kappa$ which to any

$$
s_{B}: \kappa[[t]] \longrightarrow B \longrightarrow \kappa
$$

associates the isomorphism classes of liftings of $\left(X_{0}, \lambda_{0}\right)$ to $\left(\operatorname{Spec}(B), \mathcal{M}_{B}\right)$, where $\left(X_{0}, \lambda_{0}\right)$ denotes the reduction of $(X, \lambda)$ and $\mathcal{M}_{B}$ is the $\log$ structure induced by the map $s_{B}$. It follows from the discussion following (6.11) that $D$ is pro-representable and formally smooth. Hence $D$ is pro-represented by an algebra of the form $\kappa[[t]]\left[\left[q_{1}, \ldots, q_{h}\right]\right]$. Moreover, as shown above there exists an effective universal object $(\widehat{X}, \hat{\lambda})$ over $\kappa[[t]]\left[\left[q_{1}, \ldots, q_{h}\right]\right]$, and by the full faithfulness already shown (note that the proof that (6.11.1) is fully faithful did not use the primitivity of $\lambda)$ the pair $(X, \lambda)$ is obtained by base change from $(\widehat{X}, \hat{\lambda})$ via the map $\kappa[[t]]\left[\left[q_{1}, \ldots, q_{h}\right]\right] \rightarrow \hat{A}$ induced by the reductions of $(X, \lambda)$. Thus it suffices to consider the case when $\hat{A}=\kappa[[t]]\left[\left[q_{1}, \ldots, q_{h}\right]\right]$ and $(X, \lambda)=(\hat{X}, \hat{\lambda})$. Now the condition that a polarization on a smooth (in the usual sense) K3 surface is primitive is well known to be an open and 
closed condition. Hence it suffices to show that the restriction of $\lambda$ to some smooth fiber is primitive. Considering the map

$$
\kappa[[t]]\left[\left[q_{1}, \ldots, q_{h}\right]\right] \longrightarrow \kappa[[t]], \quad q_{i} \mapsto 0
$$

we are thus reduced to the following sub-lemma.

Sub-Lemma 6.21. Let $\mathcal{M}_{\kappa[[t]]}$ be the log structure on $\operatorname{Spec}(\kappa[[t]])$ induced by $t$, and let $X /\left(\operatorname{Spec}(\kappa[[t]]), \mathcal{M}_{\kappa[[t]]}\right)$ be a special log K3 surface. Then the natural map

$$
H^{1}\left(X, \mathcal{M}_{X}^{\mathrm{gp}}\right) \longrightarrow \operatorname{Pic}\left(X_{\kappa((t))}\right)
$$

is bijective.

Proof. Since $X$ is regular, $\operatorname{Pic}\left(X_{\kappa((t))}\right)$ is the quotient of $\operatorname{Pic}(X)$ by the subgroup generated by divisors with support in the closed fiber of $X$. This is by (3.2) precisely $H^{1}\left(X, \mathcal{M}_{X}^{\mathrm{gp}}\right)$.

\subsection{Local quasi-separation condition (B.12 (iii)).}

Let $\mathcal{A}_{0}$ be as in (B.12 (iii)), and let $(X, \lambda)$ be a polarized log K3 surface over $\operatorname{Spec}\left(\mathcal{A}_{0}\right)$. Suppose given an automorphism $\sigma$ of $(X, \lambda)$ which induces the identity over a dense set of points $A_{0} \rightarrow \kappa$. Consider the open subspace $U=X-\Gamma_{\sigma}^{-1}\left(\Delta_{X_{0}}\right) \subset X_{0}$, where $\Gamma_{\sigma}$ denotes the graph of $\sigma$. Then since $\stackrel{\circ}{X}$ is flat over $\operatorname{Spec}\left(A_{0}\right)$ the image of $U$ in $\operatorname{Spec}\left(A_{0}\right)$ is an open set whose complement contains a dense set of points. Hence $U$ is the empty set which implies that $\sigma$ induces the identity on the underlying space $\stackrel{\circ}{X}$. Thus $\sigma$ is just an automorphism of $\mathcal{M}_{X}$ over $\mathcal{M}_{A_{0}}$. By (2.9), $\sigma$ must equal the identity. This completes the proof of the statement that $\mathbb{M}_{2 k}$ is an algebraic stack.

6.23. $\mathbb{M}_{2 k}$ admits an étale cover.

By ([32], 8.1), it suffices to show that the objects of $\mathbb{M}_{2 k}$ admit no infinitesimal automorphisms. Now suppose $A \rightarrow A_{0}$ is a surjective morphism with square-zero kernel and $\left(\mathcal{M}_{A}, X, \lambda\right)$ is an object of $\mathbb{M}_{2 k}$ over $\operatorname{Spec}(A)$. If $\sigma$ is an automorphism of $\left(\mathcal{M}_{A}, X, \lambda\right)$, then it follows from (2.9) that the automorphism of the underlying space of $X$ must be non-trivial. Thus the claim follows from ([18], 3.5) which shows that the underlying space of a stable log K3 surface admits no infinitesimal automorphism.

6.24. $\mathbb{M}_{2 k}$ is smooth and $\mathbb{M}_{2 k}-\mathbb{M}_{2 k}^{s m}$ is a smooth divisor with normal crossings.

By ([39], 5.14), the open substack of $\mathcal{L} o g$ classifying $\log$ structures $\mathcal{M}$ which étale locally admit a chart $\mathbb{N} \rightarrow \mathcal{M}$ is isomorphic to $\left[\mathbb{A}^{1} / \mathbb{G}_{m}\right]$ which is smooth over $\operatorname{Spec}(\mathbb{Z})$. By (6.7), $\mathbb{M}_{2 k}$ is smooth over $\left[\mathbb{A}^{1} / \mathbb{G}_{m}\right]_{\mathbb{Q}}$, and hence is smooth over $\mathbb{Q}$. Moreover, $\mathbb{M}_{2 k}-\mathbb{M}_{2 k}^{\text {sm }}$ is equal to the inverse image of the smooth divisor $B \mathbb{G}_{m} \subset\left[\mathbb{A}^{1} / \mathbb{G}_{m}\right]$ corresponding to the origin $0 \in \mathbb{A}^{1}$, and hence is a smooth divisor.

\section{Log K3 surfaces with level structure}

7.1. In this section it will be most useful to view $\mathbb{M}_{2 k}$ with its natural log structure $\mathcal{M}_{\mathbb{M}_{2 k}}$ as a stack over the category $F \log$ of fine $\log$ schemes over $\mathbb{Q}$. As remarked in (6.3), the stack $\left(\mathbb{M}_{2 k}, \mathcal{M}_{\mathbb{M}_{2 k}}\right)$ can be viewed as the stack which to any fine log scheme $T$ associates the 
groupoid of pairs $(X, \lambda)$, where $f: X \rightarrow T$ is a $\log \mathrm{K} 3$ surface and $\lambda$ is a primitive polarization of degree $2 k$.

To any such $(X, \lambda)$ and integer $n$, we obtain a sheaf $R^{2} f_{*}(\mathbb{Z} / n \mathbb{Z})$ on the log étale site of $T$ (see ([37]) for the definition of the log étale site). This sheaf is locally constant and its formation is compatible with arbitrary base change by $([36], 4.3)$ and $([37], 5.1)$. In particular, the sheaf $R^{2} f_{*}(\mathbb{Z} / n \mathbb{Z})$ is obtained by pullback from a universal sheaf $\Lambda_{n}^{\log }$ on the log étale site $\left(\mathbb{M}_{2 k}, \mathcal{M}_{\mathbb{M}_{2 k}}\right)$ log-et of $\left(\mathbb{M}_{2 k}, \mathcal{M}_{\mathbb{M}_{2 k}}\right)$. The sheaf $\Lambda_{n}^{\log }$ comes with additional structure. Indeed it follows from ([36], 2.2) that the adjunction map

$$
\Lambda_{n}^{\log } \longrightarrow R j_{*} j^{*} \Lambda_{n}^{\log }
$$

is an isomorphism, where $j: \mathbb{M}_{2 k, \text { et }}^{\text {sm }} \rightarrow\left(\mathbb{M}_{2 k}, \mathcal{M}_{\mathbb{M}_{2 k}}\right)_{\text {log-et }}$ denotes the natural morphism of topoi. The fact that $\Lambda_{n}^{\log } \simeq j_{*} j^{*} \Lambda_{n}^{\log }$ implies that the sheaf

$$
\underline{\operatorname{Isom}}\left(\Lambda \otimes \mathbb{Z} / n \mathbb{Z}, \Lambda_{n}^{\log }\right)
$$

of isomorphisms between $\Lambda_{n}^{\log }$ and the constant sheaf $\Lambda \otimes \mathbb{Z} / n \mathbb{Z}$ is also locally constant and isomorphic to

$$
j_{*} \underline{\operatorname{Isom}}\left(\Lambda \otimes \mathbb{Z} /\left.n \mathbb{Z}\right|_{\mathbb{M}_{2 k}^{s m}}, j^{*} \Lambda_{n}^{\log }\right) .
$$

7.2. Let $\mathbb{A}_{f}:=\prod_{\ell}^{\prime} \mathbb{Q}_{\ell}$ be the ring of finite adeles, and let $\mathcal{O}_{\mathbb{A}_{f}} \subset \mathbb{A}_{f}$ be the subring $\widehat{\mathbb{Z}} \simeq \prod_{\ell}^{\prime} \mathbb{Z}_{\ell}$. For any smooth (in the usual sense) polarized K3 surface $(X, \lambda)$ over an algebraically closed field $\kappa$, we get an $\mathbb{A}_{f}$-module $H^{2}\left(X, \mathbb{A}_{f}\right)$ by taking the restricted direct product over the $l$-adic cohomology groups of $X . H^{2}\left(X, \mathbb{A}_{f}\right)$ comes with the additional structure of an $\mathcal{O}_{\mathbb{A}_{f}}$-lattice $H^{2}\left(X, \mathcal{O}_{\mathbb{A}_{f}}\right)$ and an inner product (see for example the discussion in ([12], p. 18)).

Let $\Lambda$ be the lattice with inner product defined in the introduction and let $h \in \Lambda$ be a fixed vector with $h^{2}=2 k$.

Definition 7.3. A full level-n-structure on $(X, \lambda)$ is an isomorphism

$$
\eta: \Lambda / n \Lambda \simeq H^{2}\left(X_{\mathrm{et}}, \mathbb{Z} / n \mathbb{Z}\right)
$$

for which there exists a lifting $\tilde{\eta}: \Lambda \otimes \mathcal{O}_{\mathbb{A}_{f}} \simeq H^{2}\left(X, \mathcal{O}_{\mathbb{A}_{f}}\right)$ of $\eta$ which preserves inner products and maps $h$ to the class of $\lambda$. If $T$ is a scheme and $(f: X \rightarrow T, \lambda)$ is a polarized K3 surface over $T$, then a full level- $n$-structure on $(X, \lambda)$ is an isomorphism $\iota$ between the constant sheaf $\Lambda / n \Lambda$ and the sheaf $R^{2} f_{*}(\mathbb{Z} / n \mathbb{Z})$ which defines a full level- $n$-structure at each geometric point of $T$.

Lemma 7.4. Let $T$ be a $\mathbb{Q}$-scheme and let $(f: X \rightarrow T, \lambda)$ be a polarized K3 surface over $T$. For any isomorphism $\iota: \Lambda / n \Lambda \rightarrow R^{2} f_{*}(\mathbb{Z} / n \mathbb{Z})$, the condition that $\iota$ defines a full level- $n-$ structure is an open and closed condition on $T$.

Proof. The assertion is local on $T$ so we may assume that $T=\operatorname{Spec}(R)$ for some $\operatorname{ring} R$. Write $R=\lim _{\longrightarrow} R_{i}$ as a filtering inductive limit of rings of finite type over $\mathbb{Q}$, and note that since $\mathbb{M}_{2 k}^{\mathrm{sm}}$ is limit preserving by (6.4), we can by ([7], IX.2.7.4) find an approximation for the data $(X, \lambda, \iota)$ over some $R_{i}$. Thus we are reduced to the case when $T$ is locally of finite type over $\mathbb{Q}$. By base changing to $\mathbb{C}$ we see that it suffices to consider the case when $T$ is a $\mathbb{C}$-scheme of finite type, and in this case it suffices to show that the set of points where $\iota$ defines a full level- $n$-structure is open and closed in the analytic topology. Now in this case the assertion that $\iota$ defines a full level-n-structure at a point $t \in T(\mathbb{C})$ amounts to the 
statement that there exists a lifting $\tilde{\iota}: \Lambda \otimes \mathcal{O}_{\mathbb{A}_{f}} \rightarrow\left(R^{2} f_{*}^{\text {an }} \mathbb{Z}\right)_{t} \otimes \mathcal{O}_{\mathbb{A}_{f}}$ compatible with the inner products and the polarizations. This is evidently an open and closed condition since $R^{2} f_{*}^{\text {an }} \mathbb{Z}$ is a locally constant sheaf.

7.5. Define

$$
\underline{\operatorname{Isom}}^{*}\left(\Lambda /\left.n \Lambda\right|_{\mathbb{M}_{2 k}^{\mathrm{sm}}}, j^{*} \Lambda_{n}^{\log }\right) \subset \underline{\operatorname{Isom}}\left(\Lambda /\left.n \Lambda\right|_{\mathbb{M}_{2 k}^{\mathrm{sm}}}, j^{*} \Lambda_{n}^{\log }\right)
$$

to be the subsheaf of isomorphisms $\iota$ which define full level- $n$-structures. It is a locally constant sheaf by (7.4). Therefore, the sheaf

$$
\underline{\operatorname{Isom}}^{*}\left(\Lambda / n \Lambda, \Lambda_{n}^{\log }\right):=j_{*} \underline{\operatorname{Isom}}^{*}\left(\Lambda / n \Lambda, \Lambda_{n}^{\log }\right)
$$

is a locally constant subsheaf of $\underline{\operatorname{Isom}}\left(\Lambda / n \Lambda, \Lambda_{n}^{\log }\right)$.

Denote by $\operatorname{Aut}^{\mathbb{Z}}(\Lambda / n \Lambda)$ the group of automorphisms of $\Lambda / n \Lambda$ which can be lifted to automorphisms $\Lambda \simeq \Lambda$ preserving the lattices, $h$, and pairings, and let

$$
H \subset \operatorname{Aut}^{\mathbb{Z}}(\Lambda / n \Lambda)
$$

be a subgroup.

Definition 7.6. Let $T$ be a $\log$ scheme and $(f: X \rightarrow T, \lambda)$ a polarized log K3 surface over $T$. An $H$-level structure on $(X, \lambda)$ is an element of

$$
H^{0}\left(T_{\text {log-et }}, H \backslash \underline{\operatorname{Isom}}_{T_{\text {log-et }}}^{*}\left(\Lambda / n \Lambda, R^{2} f_{*}(\mathbb{Z} / n \mathbb{Z})\right)\right) .
$$

Remark 7.7. Here $\underline{\operatorname{Isom}}_{T_{\text {log-et }}}^{*}\left(\Lambda / n \Lambda, R^{2} f_{*}(\mathbb{Z} / n \mathbb{Z})\right)$ denotes the sheaf $\underline{\operatorname{Isom}}^{*}\left(\Lambda / n \Lambda, \Lambda_{n}^{\log }\right)$ pulled back to the log étale site $T_{\text {log-et }}$ of $T$ via the map $T \rightarrow\left(\mathbb{M}_{2 k}, \mathcal{M}_{\mathbb{M}_{2 k}}\right.$ ) defined by $(X, \lambda)$ (or equivalent the sheaf of full level- $n$-structures on $(X, \lambda))$. $H$ acts on this sheaf via its action on $\Lambda / n \Lambda$.

Consider the fibered category $\mathcal{F}$ over $F \log$ which to any $T$ associates the groupoid of triples $(f: X \rightarrow T, \lambda, \iota)$, where $(X, \lambda)$ is an object of $\left(\mathbb{M}_{2 k}, \mathcal{M}_{\mathbb{M}_{2 k}}\right)(T)$ and $\iota$ is an $H$-level structure on $(X, \lambda)$.

Theorem 7.8. The fibered category $\mathcal{F}$ is equivalent to that defined by a smooth DeligneMumford stack $\mathbb{M}_{2 k, H}$ with a fine log structure $\mathcal{M}_{\mathbb{M}_{2 k, H}}$. Moreover the natural map

$$
\left(\mathbb{M}_{2 k, H}, \mathcal{M}_{\mathbb{M}_{2 k, H}}\right) \longrightarrow\left(\mathbb{M}_{2 k}, \mathcal{M}_{\mathbb{M}_{2 k}}\right)
$$

is representable, finite, and log étale of Kummer type (see ([37], 2.1.2) for the definition of Kummer type). If $\mathbb{M}_{2 k, H}^{s m} \subset \mathbb{M}_{2 k, H}$ denotes the substack classifying triples $(f: X \rightarrow T, \lambda, \iota)$ with $\stackrel{\circ}{f}: \stackrel{\circ}{X} \rightarrow \stackrel{\circ}{T}$ smooth, then $\mathbb{M}_{2 k, H}-\mathbb{M}_{2 k, H}^{\mathrm{sm}}$ is a smooth divisor.

Proof. The stack $\mathcal{F}$ "is" the sheaf $H \backslash \underline{\operatorname{Isom}}^{*}\left(\Lambda / n \Lambda, \Lambda_{n}^{\log }\right)$ on the big log étale site of $\left(\mathbb{M}_{2 k}, \mathcal{M}_{\mathbb{M}_{2 k}}\right)$. This is a locally constant sheaf, and hence is representable by a relative log scheme, log étale and of Kummer type over $\left(\mathbb{M}_{2 k}, \mathcal{M}_{\mathbb{M}_{2 k}}\right)$ by a result of I. Vidal ([45], I.1.2.3). This and the following lemma proves the theorem.

Lemma 7.9. Let $X$ be a log scheme smooth over $\left(\operatorname{Spec}(\kappa), \mathcal{O}_{\kappa}^{*}\right)$ for some field $\kappa$. Assume that $\mathcal{M}_{X}$ étale locally admits a chart $\mathbb{N} \rightarrow \mathcal{M}_{X}$. Then for any Kummer-étale morphism $Y \rightarrow X$ the underlying scheme $\stackrel{\circ}{Y}$ is smooth over $\kappa$, the locus of points $y \in Y$ where $\overline{\mathcal{M}}_{Y, \bar{y}} \neq 0$ is a smooth divisor, and $\mathcal{M}_{Y}$ admits étale locally a chart $\mathbb{N} \rightarrow \mathcal{M}_{Y}$. 
Proof. By the same reasoning as in (6.24), it suffices to prove the last statement. For any geometric point $\bar{y} \rightarrow Y$, the group $\overline{\mathcal{M}}_{Y, \bar{y}}^{\mathrm{gp}}$ is by the definition of Kummer type isomorphic to $\mathbb{Z}$. Since $\overline{\mathcal{M}}_{Y, \bar{y}}$ is saturated, it follows that $\overline{\mathcal{M}}_{Y, \bar{y}}$ is isomorphic to $\mathbb{N}$. Thus we can find a map $\mathbb{N} \rightarrow \mathcal{M}_{Y, \bar{y}}$ such that the induced map $\mathbb{N} \rightarrow \overline{\mathcal{M}}_{Y, \bar{y}}$ is an isomorphism. By ([28], 2.10) this map extends to a chart in some étale neighborhood of $\bar{y}$.

7.10. Let $\Gamma \subset \Gamma_{2 k}$ be the inverse image of $H$ in $\operatorname{Aut}(\Lambda)$, and let $\mathbb{M}_{2 k, H, \mathbb{C}}$ denote the base change to $\mathbb{C}$ of $\mathbb{M}_{2 k, H}$. Consider the substack $\mathbb{M}_{2 k, \Gamma, \mathbb{C}}^{\mathrm{sm}} \subset \mathbb{M}_{2 k, H, \mathbb{C}}^{\mathrm{sm}}$ (notation explained below) whose objects over some $\mathbb{C}$-scheme $T$ consists of triples $(f: X \rightarrow T, \lambda, \iota)$ defining an object of $\mathbb{M}_{2 k, H, \mathbb{C}}^{\mathrm{sm}}(T)$ such that for each point $t \in T(\mathbb{C})$ the element

$$
\iota_{t} \in H \backslash \underline{\operatorname{Isom}}^{*}\left(\Lambda / n \Lambda, H^{2}\left(X_{t}, \mathbb{Z} / n \mathbb{Z}\right)\right)
$$

can be lifted to an isomorphism $\Lambda \simeq H^{2}\left(X_{t}^{\text {an }}, \mathbb{Z}\right)$ compatible with the polarizations and pairings. It is straightforward to verify that this is an open and closed condition so that $\mathbb{M}_{2 k, \Gamma, \mathbb{C}}^{\mathrm{sm}}$ is an open and closed substack of $\mathbb{M}_{2 k, H, \mathbb{C}}^{\mathrm{sm}}$.

7.11. The justification for the notation $\mathbb{M}_{2 k, \Gamma, \mathbb{C}}^{\mathrm{sm}}$ is as follows. To define $\mathbb{M}_{2 k, \Gamma, \mathbb{C}}^{\mathrm{sm}}$ it suffices to restrict $\mathbb{M}_{2 k, H, \mathbb{C}}$ to the category of smooth $\mathbb{C}$-schemes, since the stack $\mathbb{M}_{2 k, H, \mathbb{C}}$ is smooth. Now if $f: X \rightarrow T$ is a smooth polarized K3 surface over a smooth scheme $T$, then to give $X / T$ the structure of an object of $\mathbb{M}_{2 k, \Gamma, \mathbb{C}}^{\mathrm{sm}}$ is equivalent to giving a $\Gamma$-equivalence class of isomorphisms between the constant sheaf $\Lambda$ and $R^{2} f_{*}(\mathbb{Z})$ compatible with the polarizations and cup-product on the analytic space $T^{\text {an }}$. It follows that there is a natural map of Deligne-Mumford stacks (see the introduction for the definition of $\mathcal{D}_{2 k}$ )

$$
\mathbb{M}_{2 k, \Gamma, \mathbb{C}}^{\mathrm{sm}} \longrightarrow \Gamma \backslash \mathcal{D}_{2 k}
$$

such that the diagram

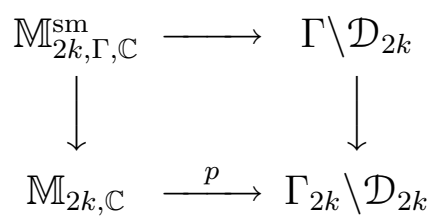

is cartesian. In particular, the map (7.11.1) is étale, surjective, and generically an isomorphism since this is true of $p$.

7.12. Let $\operatorname{Aut}^{*}(\Lambda / n \Lambda)$ denote the group of isomorphisms which can be lifted to isomorphisms $\Lambda \otimes \mathcal{O}_{\mathbb{A}_{f}} \simeq \Lambda \otimes \mathcal{O}_{\mathbb{A}_{f}}$ preserving the polarizations and pairings, and note that there is a natural inclusion

$$
\operatorname{Aut}^{\mathbb{Z}}(\Lambda / n \Lambda) \hookrightarrow \operatorname{Aut}^{*}(\Lambda / n \Lambda)
$$

The natural action of $\operatorname{Aut}^{*}(\Lambda / n \Lambda)$ on $\underline{\operatorname{Isom}}^{*}\left(\Lambda / n \Lambda, j^{*} \Lambda_{n}^{\log }\right)$ induces an action of $\operatorname{Aut}^{*}(\Lambda / n \Lambda)$ on $\mathbb{M}_{2 k, H, \mathbb{C}}$. For $g \in \operatorname{Aut}^{*}(\Lambda / n \Lambda)$, we will denote this action by $(X, \lambda, \iota) \mapsto\left(X, \lambda, \iota^{g}\right)$. Then if $g_{1}, \ldots, g_{r} \in \operatorname{Aut}^{*}(\Lambda / n \Lambda)$ denote left coset representatives for $\operatorname{Aut}^{*}(\Lambda / n \Lambda)$ modulo $\operatorname{Aut}^{\mathbb{Z}}(\Lambda / n \Lambda)$, we get an isomorphism

$$
g_{1} \coprod \cdots \coprod g_{r}: \coprod_{g_{i}} \mathbb{M}_{2 k, \Gamma, \mathbb{C}}^{\mathrm{sm}} \longrightarrow \mathbb{M}_{2 k, H, \mathbb{C}}^{\mathrm{sm}}
$$

This map extends in a natural way to an isomorphism

$$
g_{1} \coprod \cdots \coprod g_{r}: \coprod_{g_{i}} \mathbb{M}_{2 k, \Gamma, \mathbb{C}} \longrightarrow \mathbb{M}_{2 k, H, \mathbb{C}}
$$


where $\mathbb{M}_{2 k, \Gamma, \mathbb{C}}$ denotes the closure of $\mathbb{M}_{2 k, \Gamma, \mathbb{C}}^{\mathrm{sm}}$ in $\mathbb{M}_{2 k, H, \mathbb{C}}$.

7.13. This observation enables us to define a model $\mathbb{M}_{2 k, \Gamma}$ for $\mathbb{M}_{2 k, \Gamma, \mathbb{C}}$ over a canonically defined number field $E_{\Gamma}$. To do so, let $\overline{\mathbb{Q}}$ denote the algebraic closure of $\mathbb{Q}$ in $\mathbb{C}$ (so fix an embedding $\overline{\mathbb{Q}} \hookrightarrow \mathbb{C}$ ), and observe that the connected components of $\mathbb{M}_{2 k, H, \overline{\mathbb{Q}}}$ are in bijection with the connected components of $\mathbb{M}_{2 k, H, \mathbb{C}}$, so at least we get a model

$$
\mathbb{M}_{2 k, \Gamma, \overline{\mathbb{Q}}} \hookrightarrow \mathbb{M}_{2 k, H, \overline{\mathbb{Q}}}
$$

for $\mathbb{M}_{2 k, \Gamma, \mathbb{C}}$. Let $I_{\Gamma} \subset \operatorname{Gal}(\overline{\mathbb{Q}} / \mathbb{Q})$ be the subgroup of elements which map $\mathbb{M}_{2 k, \Gamma, \overline{\mathbb{Q}}}$ to itself under the natural action of $\operatorname{Gal}(\overline{\mathbb{Q}} / \mathbb{Q})$. Then $E_{\Gamma}$ is the fixed field of $I_{\Gamma}$ and $\mathbb{M}_{2 k, \Gamma}$ is the stack over $E_{\Gamma}$ obtained by descent. There is a natural closed and open immersion

$$
\mathbb{M}_{2 k, \Gamma} \hookrightarrow \mathbb{M}_{2 k, H, E_{\Gamma}} .
$$

The connected components of $\mathbb{M}_{2 k, \Gamma}$ may still not be geometrically connected. For this we introduce another field extension $F_{\Gamma} / E_{\Gamma}$. Let $J \subset \operatorname{Gal}\left(\overline{\mathbb{Q}} / E_{\Gamma}\right)$ be the subgroup of elements which preserve the decomposition of $\mathbb{M}_{2 k, \Gamma, \overline{\mathbb{Q}}}$ into connected components, and let $F_{\Gamma}$ be the fixed field of $J$. Then the connected components of $\mathbb{M}_{2 k, \Gamma, F_{\Gamma}}$ are all geometrically connected. This also implies that the irreducible components of the boundary of $\mathbb{M}_{2 k, \Gamma, F_{\Gamma}}$ are geometrically irreducible (exercise left to the reader).

Note that by construction the fields $E_{\Gamma}$ and $F_{\Gamma}$ come with specified embeddings into $\mathbb{C}$. Also the degree $\left[F_{\Gamma}: E_{\Gamma}\right]$ is either 1 or 2 . This follows from the fact that $\mathcal{D}_{2 k}$ has two connected components ([16], 0.9), and hence $\Gamma \backslash \mathcal{D}_{2 k}$ has 1 or 2 connected components. Since the natural map $\mathbb{M}_{2 k, \Gamma, \mathbb{C}}^{\mathrm{sm}} \rightarrow \Gamma \backslash \mathcal{D}_{2 k}$ is generically an isomorphism it follows that $\mathbb{M}_{2 k, \Gamma, \mathbb{C}}^{\mathrm{sm}}$ has either 1 or 2 components.

7.14. Now suppose $D \subset \mathbb{M}_{2 k, \Gamma, F_{\Gamma}}$ is a connected (and hence irreducible) component of the boundary. We will associated to $D$ a $\Gamma$-equivalence class of nilpotent endomorphisms $N$ : $\Lambda \rightarrow \Lambda$. Since $D$ remains connected and irreducible after base change via the map $F_{\Gamma} \hookrightarrow \mathbb{C}$, it suffices to associate such data to the base change $D_{\mathbb{C}}$.

Suppose $(X, \lambda, \iota)$ is a collection of data corresponding to a point in $D_{\mathbb{C}}(\mathbb{C})$. By $(7.8)$, we can find a $\log$ smooth deformation $(\tilde{X}, \tilde{\lambda}, \tilde{\iota})$ of this data to a family over the disk $\Delta$ with $\log$ structure $\mathcal{M}_{\Delta}$ defined by the function $x \in \mathbb{C}\{\{x\}\}$. Then for any point $t \in \Delta^{*}:=\Delta-\{0\}$ we obtain an automorphism $T$ of $H^{2}\left(\tilde{X}_{t}, \mathbb{Z}\right)$ by considering the path on $\Delta$ "turning once around 0 counter-clockwise". It is known that $T$ is a unipotent endomorphism and that its logarithm $N$ is an integral matrix ([20], 0.5). Now we are given a specified class

$$
\eta_{t} \in \Gamma \backslash \operatorname{Isom}\left(\Lambda, H^{2}\left(\widetilde{X}_{t}, \mathbb{Z}\right)\right),
$$

and if we choose a representative isomorphism $\tilde{\eta}_{t}: \Lambda \simeq H^{2}\left(\widetilde{X}_{t}, \mathbb{Z}\right)$ we obtain a nilpotent endomorphism of $\Lambda$. If $[N]$ denotes class of this endomorphism in the set of $\Gamma$-equivalence classes of endomorphisms of $\Lambda$, then $[N]$ is independent of the choice of $\tilde{\eta}_{t}$.

Lemma 7.15. The class $[N]$ is an invariant of the divisor $D_{\mathbb{C}}$.

Proof. To prove this we may work locally in the analytic topology on $\mathbb{M}_{2 k, \Gamma, \mathbb{C}}$. Now locally this space is isomorphic to $\Delta^{r} \times \Delta$, where $r$ is some integer, and $D_{\mathbb{C}}$ is the divisor $\Delta^{r} \times\{0\}$. Let $\mathcal{M}_{\Delta^{r} \times \Delta}$ be the $\log$ structure defined by the divisor $D_{\mathbb{C}}$. If $g:\left(\Delta, \mathcal{M}_{\Delta}\right) \rightarrow\left(\Delta^{r} \times \Delta, \mathcal{M}_{\Delta^{r} \times \Delta}\right)$ 
is any strict map and $t \in \Delta^{*}$ is a point, then the strictness implies that the induced map on fundamental groups

$$
\pi_{1}\left(\Delta^{*}, t\right) \longrightarrow \pi_{1}\left(\Delta^{r} \times \Delta^{*}, g(t)\right)
$$

is an isomorphism. Hence for any local system $V$ on $\Delta^{r} \times \Delta^{*}$, the automorphism of $V_{g(t)}$ obtained from the composite

$$
\mathbb{Z} \simeq \pi_{1}\left(\Delta^{*}, t\right) \stackrel{g_{*}}{\longrightarrow} \pi_{1}\left(\Delta^{r} \times \Delta^{*}, g(t)\right) \longrightarrow \operatorname{Aut}\left(V_{g(t)}\right)
$$

is independent of the choice of $g$. From this the lemma follows.

Definition 7.16. Let $\Sigma=\left\{\left[N_{1}\right], \ldots,\left[N_{r}\right]\right\}$ be a set of $\Gamma$-equivalence classes of endomorphisms of $\Lambda$. Define $\mathbb{M}_{2 k, \Gamma, \Sigma} \subset \mathbb{M}_{2 k, \Gamma, F_{\Gamma}}$ to be the open substack consisting of $\mathbb{M}_{2 k, \Gamma, F_{\Gamma}}^{\text {sm }}$ together with the components $D$ of the boundary whose associated $\Gamma$-equivalence class of endomorphisms of $\Lambda$ lies in $\Sigma$.

\section{Minimal and Toroidal compactifications}

Throughout this section we will work with a fixed neat arithmetic subgroup $\Gamma$ of $\Gamma_{2 k}$, and leave it for the reader to make the necessary modifications in the case when $\Gamma$ is not neat.

8.1. We begin with a discussion of the minimal compactification (sometimes also referred to the Satake or Baily-Borel compactification) of $\Gamma \backslash \mathcal{D}_{2 k}([9])$. On the analytic space $\mathcal{D}_{2 k}$, we have the universal filtration on $\Lambda \otimes \mathcal{O}_{\mathcal{D}_{2 k}}$ which in our situation is just a line $\ell \subset \Lambda \otimes_{\mathbb{C}} \mathcal{O}_{\mathcal{D}_{2 k}}$, and the action of $\Gamma$ on $\mathcal{D}_{2 k}$ extends (tautologically) to an action on these bundles. We denote by $\omega$ the bundle on $\Gamma \backslash \mathcal{D}_{2 k}$ descended from $\ell$. By $([9], 10.14), \omega$ is an algebraic bundle. Define a graded algebra $\mathfrak{A}$ by

$$
\mathfrak{A}:=\oplus_{l \geq 0} H^{0}\left(\Gamma \backslash \mathcal{D}_{2 k}, \omega^{\otimes l}\right),
$$

(here we mean algebraic sections, but in fact one can also work analytically by Koecher's principle $([9], 10.14))$.

Theorem 8.2 ([9], 10.11). The $\mathbb{C}$-algebra $\mathfrak{A}$ is finitely generated, and $\left(\Gamma \backslash \mathcal{D}_{2 k}\right)^{*}:=\operatorname{Proj}(\mathfrak{A})$ is a projective variety. Moreover, there is a natural inclusion $\left(\Gamma \backslash \mathcal{D}_{2 k}\right) \hookrightarrow\left(\Gamma \backslash \mathcal{D}_{2 k}\right)^{*}$ identifying $\Gamma \backslash \mathcal{D}_{2 k}$ with a dense open sub-scheme.

Now let

$$
\pi: \mathbb{M}_{2 k, \Gamma, \mathbb{C}}^{\mathrm{sm}} \longrightarrow \Gamma \backslash \mathcal{D}_{2 k}
$$

denote the period map (7.11.2), and notice that

$$
\pi^{*} \omega \simeq R^{0} f_{*} \Omega_{\mathcal{X}}^{2}{ }^{\mathrm{sm}} / \mathbb{M}_{2 k, \Gamma, \mathbb{C}}^{\mathrm{sm}},
$$

where $\mathcal{X}^{\mathrm{sm}}$ denotes the universal polarized K3 surface.

Proposition 8.3. For all $l \geq 0$, the map

$$
H^{0}\left(\Gamma \backslash \mathcal{D}_{2 k}, \omega^{\otimes l}\right) \longrightarrow H^{0}\left(\mathbb{M}_{2 k, \Gamma, \mathbb{C}}^{\mathrm{sm}}, \pi^{*} \omega^{\otimes l}\right)
$$

is an isomorphism. 
Proof. The map is injective since $\pi$ is generically an isomorphism. Thus we have to show that it is surjective.

Suppose we are given a global section $\eta \in \Gamma\left(\mathbb{M}_{2 k, \Gamma, \mathbb{C}}^{\mathrm{sm}}, \pi^{*} \omega^{\otimes l}\right)$. We can think of $\eta$ as a rule which to any K3 surface $f: X \rightarrow T$ over some scheme $T$ associates a global section $\eta_{X} \in \Gamma\left(T,\left(f_{*} \Omega_{X / T}^{2}\right)^{\otimes l}\right)$ such that the association $X \mapsto \eta_{X}$ is functorial. By this we mean that if $f^{\prime}: X^{\prime} \rightarrow T$ is a second polarized K3 surface with level structure, then for any isomorphism $\sigma: X \rightarrow X^{\prime}$ compatible with the polarizations and level structure, $\sigma^{*}\left(\eta_{X^{\prime}}\right)=$ $\eta_{X} \in \Gamma\left(T,\left(f_{*} \Omega_{X / T}^{2}\right)^{\otimes l}\right)$. On the other hand, to give an element in $H^{0}\left(\Gamma \backslash \mathcal{D}_{2 k}, \omega^{\otimes l}\right)$ is by the Torelli theorem and the modular interpretation given in $\Gamma \backslash \mathcal{D}_{2 k}$ equivalent to giving for each such K3 surface $f: X \rightarrow T$ a global section of $\left(f_{*} \Omega_{X / T}^{2}\right)^{\otimes l}$ which is not only compatible with isomorphisms of $\mathrm{K} 3$ surfaces but also with any isomorphism of the associated variations of Hodge structure. More precisely, if $f^{\prime}: X^{\prime} \rightarrow T$ is a second polarized K3 surface as above, and

$$
\sigma:\left(R^{2} f_{*} \mathbb{Z}, f_{*} \Omega_{X / T}^{2}, \lambda_{X}, \iota_{X}\right) \rightarrow\left(R^{2} f_{*}^{\prime} \mathbb{Z}, f_{*}^{\prime} \Omega_{X^{\prime} / T}^{2}, \lambda_{X^{\prime}}, \iota_{X^{\prime}}\right)
$$

is an isomorphism of variations of Hodge structure compatible with all the additional structure, then $\sigma\left(\eta_{X}\right)=\eta_{X^{\prime}}$. Now to verify that any $\eta \in H^{0}\left(\mathbb{M}_{2 k, \Gamma, \mathbb{C}}^{\mathrm{sm}}, \pi^{*} \omega^{\otimes l}\right)$ has this stronger property, we may assume that $X \rightarrow T$ and $X^{\prime} \rightarrow T$ are suitably versal families. In this case the result is clear, because we know that there exists a dense open set $U \subset T$ over which $\sigma$ is induced by an actual isomorphism of polarized K3 surfaces with additional structure, by the Torelli theorem ([16], 1.2).

If $f: \mathcal{X} \rightarrow \mathbb{M}_{2 k, \Gamma, \mathbb{C}}$ denotes the universal $\log \mathrm{K} 3$ surface with polarization, and if $\Omega_{\mathcal{X} / \mathbb{M}_{2 k, \Gamma, \mathbb{C}}}^{1}$ denotes the sheaf of $\log$ differential forms on $\mathcal{X} / \mathbb{M}_{2 k, \Gamma, \mathbb{C}}$, then we obtain the following:

Corollary 8.4. The natural map

$$
H^{0}\left(\mathbb{M}_{2 k, \Gamma, \mathbb{C}},\left(f_{*} \Omega_{\mathcal{X} / \mathbb{M}_{2 k, \Gamma, \mathbb{C}}}\right)^{\otimes l}\right) \longrightarrow H^{0}\left(\mathbb{M}_{2 k, \Gamma, \mathbb{C}}^{\mathrm{sm}}, \pi^{*} \omega^{\otimes l}\right)
$$

is an isomorphism, and for l sufficiently big $\left(f_{*} \Omega_{\mathcal{X} / \mathbb{M}_{2 k, \Gamma, \mathbb{C}}}^{2}\right)^{\otimes l}$ is generated by global sections.

Proof. Since $\mathbb{M}_{2 k, \Gamma, \mathbb{C}}$ is smooth and $\mathbb{M}_{2 k, \Gamma, \mathbb{C}}^{\mathrm{sm}}$ is a dense open subset of $\mathbb{M}_{2 k, \Gamma, \mathbb{C}}$, the map is injective. Thus it suffices to show that every section of $\pi^{*} \omega^{\otimes l}$ has at most logarithmic poles along the boundary. This assertion is local on $\mathbb{M}_{2 k, \Gamma, \mathbb{C}}$, and we may even work locally in the analytic topology. Thus it suffices to consider the situation arising from a family over $\Delta^{r} \times \Delta$ whose restriction to $\Delta^{r} \times \Delta^{*}$ is smooth. We may also assume that the map $\Delta^{r} \times \Delta^{*} \rightarrow \Gamma \backslash \mathcal{D}_{2 k}$ is a local isomorphism. In this case it follows from ([8], p. 279) that the period map can be extended to a map from $\Delta^{r} \times \Delta$ to one of Mumfords toroidal compactifications. This implies that the map is surjective, for it is known that any section of $H^{0}\left(\Gamma \backslash \mathcal{D}_{2 k}, \omega^{\otimes l}\right)$ extends to a section over any of the toroidal compactifications by ([9], 10.14) and ([8], p. 254).

The same argument yields the second assertion. For it follows from the construction of the minimal compactification that for some integer $l$ the sheaf $\omega^{\otimes l}$ extends to all of $\left(\Gamma \backslash \mathcal{D}_{2 k}\right)^{*}$ and is generated by global sections. Since all the toroidal compactifications map to the minimal compactification the above argument yields the result.

Corollary 8.5. The period map $\mathbb{M}_{2 k, \Gamma, \mathbb{C}}^{\mathrm{sm}} \rightarrow \Gamma \backslash \mathcal{D}_{2 k}$ extends naturally to a map

$$
\pi: \mathbb{M}_{2 k, \Gamma, \mathbb{C}} \longrightarrow\left(\Gamma \backslash \mathcal{D}_{2 k}\right)^{*} \text {. }
$$


8.6. Next we turn our attention to the toroidal compactifications of Mumford et. al. ([8]).

Recall that a toroidal compactification of $\Gamma \backslash \mathcal{D}_{2 k}$ depends on the choice of a so-called " $\Gamma$ admissible collection of fans" $\Delta=\left(\Delta_{F}\right)$; one for each "rational boundary component" ([8], III.3.5). Each $\Delta_{F}$ is a fan in a certain unipotent subgroup $U^{F}$ of the group of automorphisms of $\Lambda \otimes \mathbb{R}$ compatible with the inner product and the polarization, and the fans satisfy certain compatibilities. Here $U^{F}$ is given the structure of a vector space by identifying $U^{F}$ with its Lie algebra using the logarithm map. For any one-dimensional cone $\sigma$ in some $\Delta_{F}$ we obtain a cone in $U^{F}$ which we always assume to be integral. That is, viewing $U^{F}$ as a subspace of $\operatorname{End}\left(\Lambda_{\mathbb{R}}\right)$, we assume that $\sigma \cap \operatorname{End}(\Lambda)$ is non-zero and generated by a single element $N_{\sigma}$. Thus to any $\Delta$ we associate a set of $\Gamma$-equivalence class of endomorphisms of $\Lambda$. We denote this collection by $\Sigma(\Delta)$, or just $\Sigma$ if no confusion is likely to arise.

We will always in this paper assume that $\Delta$ is "smooth" so that the associated toroidal compactification $\overline{\left(\Gamma \backslash \mathcal{D}_{2 k}\right)}$ is a smooth algebraic space with boundary a divisor with normal crossings ([8], p. 293). The divisors of the boundary are in natural bijection with the $\Gamma$ equivalence classes of one-dimensional cones $\sigma$ in the $\Delta_{F}$.

Let us fix a $\Gamma$-admissible collection of fans $\Delta$ and let $\overline{\left(\Gamma \backslash \mathcal{D}_{2 k}\right)}$ denote the associated toroidal compactification. There is a natural map

$$
\operatorname{pr}: \overline{\left(\Gamma \backslash \mathcal{D}_{2 k}\right)} \longrightarrow\left(\Gamma \backslash \mathcal{D}_{2 k}\right)^{*}
$$

to the minimal compactification ([8], p. 254). We will assume that $\Delta$ is a so-called "smooth projective" collection of fans ([8], p. 310). In this case, $\overline{\left(\Gamma \backslash \mathcal{D}_{2 k}\right)}$ is a smooth algebraic variety, and the map (8.6.1) can be constructed as follows ([8], chapter IV). This construction depends on the choice of a so-called polarizing function which determines an invertible sheaf of ideals $I$ on $\overline{\left(\Gamma \backslash \mathcal{D}_{2 k}\right)}$ ([8], p. 312). This ideal $I$ has support on the boundary of $\overline{\left(\Gamma \backslash \mathcal{D}_{2 k}\right)}$, and is determined by its order of vanishing $l_{[\sigma]}$ on each divisor on the boundary corresponding to a $\Gamma$-equivalence class $[\sigma]$ of one-dimensional cones in $\Delta$. Let $J=\operatorname{pr}_{*} I$. Then $\overline{\left(\Gamma \backslash \mathcal{D}_{2 k}\right)}$ is obtained from $\left(\Gamma \backslash \mathcal{D}_{2 k}\right)^{*}$ by blowing up the ideal $J$ and normalizing. The main point of this section is that the ideal $J$ may be constructed using the stack $\mathbb{M}_{2 k, \Gamma, \Sigma}$ instead of $\overline{\left(\Gamma \backslash \mathcal{D}_{2 k}\right)}$, and that $J$ will be defined over the field $F_{\Gamma}$.

In fact, define $I_{\mathbb{M}}$ to be the sheaf of ideals on $\mathbb{M}_{2 k, \Gamma, \Sigma}$ associated to the divisor $\sum_{\sigma} l_{[\sigma]} D_{[\sigma]}$, where $D_{[\sigma]}$ denotes the divisor on the boundary of $\mathbb{M}_{2 k, \Gamma, \Sigma}$ corresponding to the class $[\sigma]$.

Proposition 8.7. Over $\mathbb{C}, J \simeq \pi_{*} I_{\mathbb{M}}$, and $\pi^{*} \pi_{*} I_{\mathbb{M}}=I_{\mathbb{M}}$.

Proof. By ([20], 6.2) and ([16], 3.8) there exists locally in the analytic topology a factorization of the map $\mathbb{M}_{2 k, \Gamma, \Sigma} \otimes \mathbb{C} \rightarrow\left(\Gamma \backslash \mathcal{D}_{2 k}\right)^{*}$ through an étale map to $\overline{\left(\Gamma \backslash \mathcal{D}_{2 k}\right)}$. From this it follows that there exists a morphism in the analytic category $\mathbb{M}_{2 k, \Gamma, \Sigma} \otimes \mathbb{C} \rightarrow \overline{\left(\Gamma \backslash \mathcal{D}_{2 k}\right)}$ (which we will see below is in fact algebraic) covering the maps to $\left(\Gamma \backslash \mathcal{D}_{2 k}\right)^{*}$. By $([44]$, theorem 2) the image of this map meets every irreducible component of the boundary. Thus a function $f \in \mathcal{O}_{\left(\Gamma \backslash \mathcal{D}_{2 k}\right)^{*}}$ vanishes with the correct multiplicity along each component of the boundary if and only if $f$ is in $\operatorname{pr}_{*} I=J$. From this it follows that $\pi_{*} I_{\mathbb{M}}=J$. The same argument combined with the fact that $\operatorname{pr}^{*} \operatorname{pr}_{*} I=I\left([8]\right.$, IV proposition 5) implies that $\pi^{*} \pi_{*} I_{\mathbb{M}}=I_{\mathbb{M}}$. 
By the universal property of blowing up and normalization we obtain a map

$$
\tilde{\pi}: \mathbb{M}_{2 k, \Gamma, \Sigma} \otimes \mathbb{C} \longrightarrow \overline{\left(\Gamma \backslash \mathcal{D}_{2 k}\right)} \text {. }
$$

This map is étale, for this can be verified locally in the analytic topology in which case the result follows from ([16], 4.2.4) and ([20], 3.8 and 6.2).

Proposition 8.8. (i) $\mathbb{M}_{2 k, \Gamma, \Sigma} \otimes \mathbb{C}$ is an algebraic space with separated diagonal, and $\tilde{\pi}$ is quasi-finite.

(ii) If $V \subset \overline{\left(\Gamma \backslash \mathcal{D}_{2 k}\right)}$ denotes the image of $\tilde{\pi}$, then $\overline{\left(\Gamma \backslash \mathcal{D}_{2 k}\right)}-V$ has codimension $\geq 2$, and the map $\mathbb{M}_{2 k, \Gamma, \Sigma} \otimes \mathbb{C} \rightarrow V$ induced by $\tilde{\pi}$ is quasi-finite and universal for maps from $\mathbb{M}_{2 k, \Gamma, \Sigma,} \otimes \mathbb{C}$ to separated algebraic spaces.

Proof. Statement (i) follows from the following three lemmas.

Lemma 8.9. The diagonal $\Delta: \mathbb{M}_{2 k} \rightarrow \mathbb{M}_{2 k} \times \mathbb{M}_{2 k}$ satisfies the valuative criterion for separatedness.

Proof. This is equivalent to saying that if $A$ is an integral domain with a log structure $\mathcal{M}_{A}$ and $\left(X, \mathcal{M}_{X}\right) /\left(A, \mathcal{M}_{A}\right)$ is a special log K3 surface, then any automorphism $\sigma$ of the corresponding $A$-valued point of $\mathbb{M}_{2 k}$ which is generically the identity is in fact equal to the identity. This is an étale local issue on $\operatorname{Spec}(A)$ so we may assume we have a chart $\mathbb{N} \rightarrow A, 1 \mapsto t$, for $\mathcal{M}_{A}$. The automorphism $\sigma$ induces an automorphism of $\mathcal{M}_{A}$ which is the same as giving a unit $u \in A^{*}$ such that $u t=t$. Since $A$ is an integral domain, $u=1$. The lemma now follows from the argument given in (6.22).

Lemma 8.10. Let $\mathcal{X}$ be a Deligne-Mumford stack which admits an étale morphism $f: X \rightarrow Y$ to an algebraic space $Y$. Then the automorphism space $\underline{\operatorname{Aut}}(x)$ of any object $x \in \mathcal{X}$ is smooth. In particular, if $f$ is generically an isomorphism and if the diagonal of $\mathcal{X}$ satisfies the valuative criterion for separatedness, then $\mathcal{X}$ is an algebraic space.

Proof. Let $\kappa$ be an algebraically closed field, $x \in \mathcal{X}(\kappa)$ an object, and let $\widehat{\mathcal{O}}_{x, x}$ be the completion of the local ring of $\mathcal{X}$ at $x$. If $\hat{x} \in \mathcal{X}\left(\widehat{\mathcal{O}}_{x, x}\right)$ denotes the tautological object, then we show that every automorphism of $x$ can be lifted to an automorphism of $\hat{x}$.

The ring $\widehat{\mathcal{O}}_{x, x}$ can also be viewed as the ring pro-representing the functor of deformations of $x$. Let $\sigma_{0}:\left.\hat{x}\right|_{\kappa} \simeq x$ denote the specified isomorphism. If $\alpha$ is an automorphism of $x$, then we obtain a formal deformation $\hat{x}^{\prime}$ by considering the object $\hat{x}$ over $\widehat{\mathcal{O}}$ together with the isomorphism $\alpha \circ \sigma_{0}:\left.\hat{x}\right|_{\kappa} \simeq x$. By the universal property there exists a unique isomorphism $\iota: \widehat{\mathcal{O}} \rightarrow \widehat{\mathcal{O}}$ and an isomorphism $\hat{\alpha}: \iota^{*} \hat{x} \simeq \hat{x}$ inducing $\alpha$. On the other hand, since the map $f: X \rightarrow Y$ is étale, the ring $\widehat{\mathcal{O}}$ is isomorphic to $\widehat{\mathcal{O}}_{Y, f(x)}$. The above construction of the action of $\underline{\operatorname{Aut}}(x)(\kappa)$ on $\widehat{\mathcal{O}}$ is functorial and so the induced action on $\widehat{\mathcal{O}}_{Y, f(x)}$ is just the action of $\underline{\operatorname{Aut}}(f(x))(\kappa)=\{i d\}$. Thus the action of $\underline{\operatorname{Aut}}(x)(\kappa)$ on $\widehat{\mathcal{O}}$ is trivial, and the automorphism $\alpha$ can be lifted to an automorphism of $\hat{x}$. This implies that Aut $(x)$ is smooth.

Now suppose the diagonal of $\mathcal{X}$ satisfies the valuative criterion for separatedness and that $f$ is generically an isomorphism. Since the Aut-spaces are smooth, to verify that they are all trivial we may base change to $Y_{\text {red }}$ and hence can assume that $\mathcal{X}$ and $Y$ are reduced. Moreover, it suffices to show that any automorphism $\sigma$ of an object $x \in \mathcal{X}$ over a separably 
closed field $\kappa$ is trivial. Since $\kappa$ is separably closed, there exists an étale morphism $u: U \rightarrow X$ and a lifting $\tilde{x}: \operatorname{Spec}(\kappa) \rightarrow U$ of $x$. Since $\operatorname{Aut}(u)$ is smooth, we can after replacing $U$ by an étale neighborhood of $\tilde{x}$ lift the automorphism $\sigma$ to an automorphism $\tilde{\sigma}$ of $u$. We claim that $\tilde{\sigma}$ is trivial. To verify this we may base change to the irreducible components of $U$ and hence may assume that $U$ is integral. Since $\tilde{\sigma}$ is generically trivial by assumption it follows from the fact that $\underline{\operatorname{Aut}}(u)$ satisfies the valuative criterion for separatedness that $\tilde{\sigma}$ is trivial.

Lemma 8.11. The map $\tilde{\pi}$ is quasi-finite.

Proof. It suffices to consider the fibers of $\tilde{\pi}$ over points $P=\operatorname{Spec}(\mathbb{C}) \rightarrow \overline{\left(\Gamma \backslash \mathcal{D}_{2 k}\right)}$ on the boundary. Suppose $\left(X_{i}, \lambda_{i}, \iota_{i}\right)(i=1,2)$ are two points of $\mathbb{M}_{2 k, \Gamma, \Sigma}$ in the fiber over $P$.

Choose a representative line bundle $\mathcal{L}_{1}$ of $\lambda_{1}$ such $\mathcal{L}_{1}^{N}$ is generated by global sections and defines a map $\stackrel{\circ}{X}_{1} \rightarrow \bar{X} \subset \mathbb{P}^{r}$ contracting finitely many curves to points.

Choose a map $\operatorname{Spec}(\mathbb{C}[[t]]) \rightarrow \overline{\left(\Gamma \backslash \mathcal{D}_{2 k}\right)}$ mapping the closed point to $P$ and the generic point to a point of $\Gamma \backslash \mathcal{D}_{2 k}$ in the open set over which $\tilde{\pi}$ is an isomorphism. Since $\tilde{\pi}$ is étale, we can lift this map to two maps $\rho_{i}: \operatorname{Spec}(\mathbb{C}[[t]]) \rightarrow \mathbb{M}_{2 k, \Gamma, \Sigma} \otimes \mathbb{C}$ corresponding to deformations $\left(\tilde{X}_{i}, \tilde{\lambda}_{i}, \tilde{\iota}_{i}\right) \in \mathbb{M}_{2 k, \Gamma, \Sigma} \otimes \mathbb{C}$ of $\left(X_{i}, \lambda_{i}, \iota_{i}\right)$ which are generically isomorphic. Moreover, by (6.8), we can find a representative $\widetilde{\mathcal{L}}_{1}$ of $\tilde{\lambda}_{1}$ lifting $\mathcal{L}_{1}$ such that $\widetilde{\mathcal{L}}_{1}^{N}$ is generated by global sections and defines a birational map $\tilde{X}_{1} \rightarrow Y \subset \mathbb{P}_{\operatorname{Spec}(\mathbb{C}[t]])}^{r}$ which in each fiber contracts only finitely many curves. Let $g: \tilde{X}_{1} \rightarrow \tilde{X}_{2}$ by the birational map obtained from the fact that the the two points $\rho_{i}(\mathbb{C}((t)))$ are equal. By the proof of $([44], 3.1)$, the map $g$ is a composite of elementary modifications ([19], 12-14) along curves in the closed fiber contracted by $\mathcal{L}_{1}^{N}$. Moreover, by (6.21) the polarization $\lambda_{2}$ is that induced by the strict transform of $\lambda_{1}$, and $\iota_{2}$ is the level structure induced by $\iota_{1}$ since they agree on the generic fiber. Now since $\mathcal{L}_{1}^{N}$ contracts only finitely many curves forming configurations as in (5.16), the set of possible birational modifications of $X_{1}$ which one can make using these curves is a finite set.

To see (ii), consider the closed subspace

$$
\mathbb{M}_{2 k, \Gamma, \Sigma} \otimes \mathbb{C} \times{ }_{V} \mathbb{M}_{2 k, \Gamma, \Sigma} \otimes \mathbb{C} \subset \mathbb{M}_{2 k, \Gamma, \Sigma} \otimes \mathbb{C} \times \mathbb{M}_{2 k, \Gamma, \Sigma} \otimes \mathbb{C}
$$

It is the étale equivalence relation on $\mathbb{M}_{2 k, \Gamma, \Sigma} \otimes \mathbb{C}$ which defines $V$. On the other hand, the fact that $\tilde{\pi}$ is generically an isomorphism implies that $\mathbb{M}_{2 k, \Gamma, \Sigma} \otimes \mathbb{C} \times_{V} \mathbb{M}_{2 k, \Gamma, \Sigma} \otimes \mathbb{C}$ is equal to the closure $\bar{\Delta}$ of the diagonal $\Delta$ of $\mathbb{M}_{2 k, \Gamma, \Sigma} \otimes \mathbb{C}$. Now if $\mathbb{M}_{2 k, \Gamma, \Sigma} \otimes \mathbb{C} \rightarrow T$ is any map to a separated algebraic space, the inverse image of the diagonal $\Delta_{T}$ of $T$ in $\mathbb{M}_{2 k, \Gamma, \Sigma} \otimes \mathbb{C} \times \mathbb{M}_{2 k, \Gamma, \Sigma} \otimes \mathbb{C}$ is closed, and hence the map

$$
\mathbb{M}_{2 k, \Gamma, \Sigma} \otimes \mathbb{C} \times{ }_{V} \mathbb{M}_{2 k, \Gamma, \Sigma} \otimes \mathbb{C} \longrightarrow T \times T
$$

factors through $\Delta_{T}$. From this it follows that $\mathbb{M}_{2 k, \Gamma, \Sigma} \otimes \mathbb{C} \rightarrow T$ factors through $V$.

Since $I_{\mathbb{M}}$ is defined over $F_{\Gamma}$, the ideal $J$ is also defined over $F_{\Gamma}$ and we obtain the following:

Corollary 8.12. Define ${\overline{\left(\Gamma \backslash \mathcal{D}_{2 k}\right)}}_{F_{\Gamma}}$ to be the normalization of the blow-up of $J:=\pi_{*} I_{\mathbb{M}}$ on $\left(\Gamma \backslash \mathcal{D}_{2 k}\right)^{*}$ (over $\left.F_{\Gamma}\right)$. Then all the assertions of (8.8) are valid over $F_{\Gamma}$ when we replace $\overline{\left(\Gamma \backslash \mathcal{D}_{2 k}\right)}$ by $\overline{\left(\Gamma \backslash \mathcal{D}_{2 k}\right)} F_{\Gamma}$ and $\mathbb{M}_{2 k, \Gamma, \Sigma} \otimes \mathbb{C}$ by $\mathbb{M}_{2 k, \Gamma, \Sigma}$ in the statements. 
8.13. Finally we remark that the various naturally occuring sheaves on $\mathbb{M}_{2 k, \Gamma, \Sigma}$ descend to ${\overline{\left(\Gamma \backslash \mathcal{D}_{2 k}\right)}}_{F_{\Gamma}}$. More precisely, let $f: \mathcal{X} \rightarrow \mathbb{M}_{2 k, \Gamma, \Sigma}$ be the universal log K3 surface. Denote by $\left(R^{2} f_{*} \Omega_{\mathcal{X} / \mathbb{M}_{2 k, \Gamma, \Sigma}}, \nabla\right)$ the relative logarithmic de Rham-cohomology of $\mathcal{X}$, and let $F^{\bullet}$ be the Hodge filtration on $R^{2} f_{*} \Omega_{\mathcal{X} / \mathbb{M}_{2 k, \Gamma, \Sigma}}$.

Proposition 8.14. The triple $\left(R^{2} f_{*} \Omega_{\mathcal{X} / \mathbb{M}_{2 k, \Gamma, \Sigma}}, \nabla, F^{\bullet}\right)$ is obtained by pullback from a unique triple $\left(\mathcal{H}, \nabla, F^{\bullet}\right)$ over $\overline{\left(\Gamma \backslash \mathcal{D}_{2 k}\right)} F_{\Gamma}$.

Proof. Let us first consider the situation over $\mathbb{C}$. On $\Gamma \backslash \mathcal{D}_{2 k}$ we have a tautological module with integrable connection $(\mathcal{H}, \nabla) .(\mathcal{H}, \nabla)$ has unipotent monodromy along the divisor at infinity since this can be verified on $\mathbb{M}_{2 k, \Gamma, \Sigma}$. Thus there is a unique extension of $(\mathcal{H}, \nabla)$ to a module with logarithmic connection on $\left(\overline{\Gamma \backslash \mathcal{D}_{2 k}}\right)$ (the canonical extension in the sense of ([11], p. 92)). By the uniqueness of the extension this pair $(\mathcal{H}, \nabla)$ bulls back to $\left(R^{2} f_{*} \Omega_{X / \mathbb{M}_{2 k, \Gamma, \Sigma}}, \nabla\right)$ on $\mathbb{M}_{2 k, \Gamma, \Sigma} \otimes \mathbb{C}$. The canonical global section $\eta \in \mathcal{H}$ over $\Gamma \backslash \mathcal{D}_{2 k}$ extends to a global section over $\left(\overline{\Gamma \backslash \mathcal{D}_{2 k}}\right)$ since this can be verified on an étale cover of an open set $U \subset\left(\overline{\Gamma \backslash \mathcal{D}_{2 k}}\right)$ whose complement has codimension $\geq 2$ (in our case $\mathbb{M}_{2 k, \Gamma, \Sigma} \otimes \mathbb{C}$ ). By the same reasoning, the cup-product pairing on $\mathcal{H}$ over $\Gamma \backslash \mathcal{D}_{2 k}$ can be extended to a pairing on $\mathcal{H}$ over all of $\left(\overline{\Gamma \backslash \mathcal{D}_{2 k}}\right)$. Now once we have the cup-product pairing on $\mathcal{H}$, to give the Hodge filtration on $\mathcal{H}$ it suffices to give the line $F^{2}$ since $F^{1}$ is the dual of $F^{2}$. Since we have such a universal line over the minimal compactification we obtain an invertible sheaf over $\left(\overline{\Gamma \backslash \mathcal{D}_{2 k}}\right)$. To extend the inclusion $F^{2} \hookrightarrow \mathcal{H}$ over $\Gamma \backslash \mathcal{D}_{2 k}$ to all of $\left(\overline{\Gamma \backslash \mathcal{D}_{2 k}}\right)$ it again is enough to work in an étale neighborhood of a subset whose complement has codimension $\geq 2$. Thus we also obtain the Hodge filtration $F^{\bullet}$ on $\mathcal{H}$. This completes the proof over $\mathbb{C}$.

Lemma 8.15. Let $\mathcal{E}$ be a locally free sheaf on $\left(\overline{\Gamma \backslash \mathcal{D}_{2 k}}\right)$. Then the natural map $\mathcal{E} \rightarrow \tilde{\pi}_{*} \tilde{\pi}^{*} \mathcal{E}$ is an isomorphism.

Proof. Since $\tilde{\pi}$ is generically an isomorphism the map is injective. To see that it is surjective, it suffices to show that if $U \rightarrow\left(\overline{\Gamma \backslash \mathcal{D}_{2 k}}\right)$ is étale, and if $U_{\mathbb{M}}:=U \times_{\left(\overline{\Gamma \backslash \mathcal{D}_{2 k}}\right)} \mathbb{M}_{2 k, \Gamma, \Sigma} \otimes \mathbb{C}$, then any section of $\mathcal{E}$ over $U_{\mathbb{M}}$ maps to the same section of $\mathcal{E}$ over $U_{\mathbb{M}} \times_{U} U_{\mathbb{M}}$ via the two projections. For since the image of $U_{\mathbb{M}} \rightarrow U$ has complement of codimension $\geq 2$, any section of $\mathcal{E}$ over $U_{\mathbb{M}}$ with descent datum relative to $U_{\mathbb{M}} \rightarrow U$ will descend to a section over $U$. Thus the result follows from the fact that over some dense open subset of $U_{\mathbb{M}}$, the two maps $U_{\mathbb{M}} \times_{U} U_{\mathbb{M}} \rightrightarrows U_{\mathbb{M}}$ are the same and open immersions (since $U_{\mathbb{M}} \rightarrow U$ is generically an isomorphism).

The lemma enables to construct $\left(\mathcal{H}, \nabla, F^{\bullet}\right)$ over $F_{\Gamma}$. Indeed the lemma implies that the sheaves $\tilde{\pi}_{*} R^{2} f_{*} \Omega_{\mathcal{X} / \mathbb{M}_{2 k, \Gamma, \Sigma}}$ and $\tilde{\pi}_{*} F^{i}$ on $\left(\overline{\Gamma \backslash \mathcal{D}_{2 k}}\right)_{F_{\Gamma}}$ induce the sheaf $\mathcal{H}$ with its Hodge filtration over $\mathbb{C}$. Thus by descent theory these sheaves are locally free. Moreover, since $\tilde{\pi}_{*} \tilde{\pi}^{*} \Omega_{\left(\overline{\Gamma \backslash \mathcal{D}_{2 k}}\right)_{F_{\Gamma}}}^{1} \simeq \Omega_{\left(\overline{\Gamma \backslash \mathcal{D}_{2 k}}\right)_{F_{\Gamma}}}^{1}$, and $\tilde{\pi}^{*} \Omega_{\left(\overline{\Gamma \backslash \mathcal{D}_{2 k}}\right)_{F_{\Gamma}}}^{1}=\Omega_{\mathbb{M}_{2 k, \Gamma, \Sigma}}^{1}$, we see that the Gauss-Manin connection induces a connection on $\tilde{\pi}_{*} R^{2} f_{*} \Omega_{\mathcal{X} / \mathbb{M}_{2 k, \Gamma, \Sigma}}$. Thus we define $\left(\mathcal{H}, \nabla, F^{\bullet}\right)$ simply to be the pushforward via $\tilde{\pi}$ of the triple $\left(R^{2} f_{*} \Omega_{\mathcal{X} / \mathbb{M}_{2 k, \Gamma, \Sigma}}, \nabla, F^{\bullet}\right)$. 


\section{Appendix A. A relative version of M. Artin's method}

A.1. In this section we prove a version of Artin's theorem ([2], theorem 5.3) with an algebraic stack as a base. Our motivation for this is the following. In our study of degenerate K3 surfaces, we have considered a stack $F$ whose objects over a scheme $T$ are triples $\left(\mathcal{M}_{T}, X, \lambda\right)$, where $\mathcal{M}_{T}$ is a $\log$ structure on $T, X /\left(T, \mathcal{M}_{T}\right)$ is a $\log \mathrm{K} 3$ surface, and $\lambda$ is a polarization (see section 5 for definitions). We would like to conclude that $F$ is algebraic. Since the deformation theory of log algebraic spaces is well-understood and the notion of polarization depends on the log structure, it is most natural to view this situation via the functor

$$
F \longrightarrow \mathcal{L} o g, \quad\left(\mathcal{M}_{T}, X, \lambda\right) \mapsto \mathcal{M}_{T}
$$

Thus we are naturally led to look for a version of Artin's theorem with an algebraic stack as a base.

Let $S$ be a scheme of finite type over an excellent Dedekind ring, and let $\mathcal{S} \rightarrow S$ be an algebraic stack locally of finite type.

Definition A.2. An $\mathcal{S}$-stack is a stack $F$ over the category of $S$-schemes with the étale topology together with a morphism of stacks $m: F \rightarrow \mathcal{S}$ (we often write simply $F$ instead of $(F, m))$. A morphism of $\mathcal{S}$-stacks $F_{1} \rightarrow F_{2}$ is a morphism of stacks $\phi: F_{1} \rightarrow F_{2}$ together with an isomorphism of functors $\psi: m_{2} \circ \phi \simeq m_{1}$. An $\mathcal{S}$-stack $(F, m)$ is algebraic if $F$ is an algebraic stack. Viewing schemes as stacks we get notions of $\mathcal{S}$-schemes and morphisms of $\mathcal{S}$-schemes.

A.3. Fix an $\mathcal{S}$-stack $(F, m)$. If $X$ is an $\mathcal{S}$-scheme define a groupoid $F_{X}$ as follows. The objects of $F_{X}$ are morphisms of $\mathcal{S}$-stacks $X \rightarrow F$. If $\left(f, f^{b}\right)$ and $\left(f^{\prime}, f^{\prime b}\right)$ are two objects of $F_{X}$, then a morphism

$$
\left(f^{\prime}, f^{\prime b}\right) \longrightarrow\left(f, f^{b}\right)
$$

in $F_{X}$ is an isomorphism of functors $\alpha: f^{\prime} \rightarrow f$ such that the two isomorphisms

$$
f^{\prime b}, f^{b} \circ \alpha: m \circ f^{\prime} \longrightarrow x
$$

are equal, where $x: X \rightarrow \mathcal{S}$ denotes the structure morphism. Since $F$ is a fibered category, $F_{X}$ is a groupoid. If $(\phi, \psi): X^{\prime} \rightarrow X$ is a morphism of $\mathcal{S}$-schemes, then there is a natural pullback functor

$$
(\phi, \psi)^{*}: F_{X} \longrightarrow F_{X^{\prime}}
$$

which sends a pair $\left(f, f^{b}\right)$ to $\left(\phi^{*}(f), \psi \circ \phi^{*}\left(f^{b}\right)\right)$. In this way $F$ defines a fibered category over the category of $\mathcal{S}$-schemes.

If $a \in F_{X^{\prime}}$ we denote by $F_{a, X}$ the category whose objects are pairs $\left(a^{\prime}, \alpha\right)$ where $a^{\prime} \in F_{X}$ and $\alpha:(\phi, \psi)^{*} a^{\prime} \simeq a$ is an isomorphism in $F_{X^{\prime}}$. Morphisms in $F_{a, X}$ are defined to be morphisms in $F_{X}$ which are compatible with the morphisms to $a$. We denote by $\bar{F}_{a, X}$ the isomorphism classes of objects in $F_{a, X}$.

A.4. The category of $\mathcal{S}$-schemes has an étale topology. Covering families $\left\{U_{i} \rightarrow U\right\}$ are collections of morphisms of $\mathcal{S}$-schemes $U_{i} \rightarrow U$ such that the underlying family of morphisms of schemes $\left\{U_{i} \rightarrow U\right\}$ is an étale cover of $U$. The condition that $F$ is a stack with respect to the étale topology on $S$-schemes is equivalent to the statement that the corresponding fibered category over the category of $\mathcal{S}$-schemes is a stack with respect to this topology (details left to reader). 
A.5. By a $\mathcal{S}$-ring $\mathcal{A}$ we mean a ring $A$ together with a structure of a $\mathcal{S}$-scheme on $\operatorname{Spec}(A)$. We usually denote $\mathcal{S}$-rings by script letters (e.g. $\mathcal{A}$ ), and their underlying rings by the corresponding roman letter (e.g. $A$ ). It follows from the fact that $\mathcal{S}$ is locally of finite type over $S$ that the stack $F$ is limit preserving (in the sense of ([2], p. 167) if and only if for all filtering inductive limits of $\mathcal{S}$-rings $\left\{\mathcal{A}_{i}\right\}$ the natural functor

$$
\stackrel{\lim }{\longrightarrow} F_{\mathcal{A}_{i}} \longrightarrow F_{\underline{\lim } \mathcal{A}_{i}}
$$

is an equivalence of categories.

Definition A.6. An $\mathcal{S}$-deformation situation is a diagram in the category of $\mathcal{S}$-rings

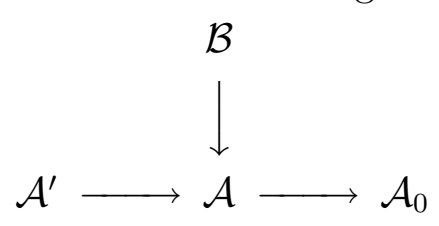

such that

(i) $A_{0}$ is reduced.

(ii) $A^{\prime} \rightarrow A$ and $A \rightarrow A_{0}$ are surjections with nilpotent kernel.

(iii) The composite $B \rightarrow A_{0}$ is surjective.

(iv) The kernel $I=\operatorname{Ker}\left(A^{\prime} \rightarrow A\right)$ is an $A_{0}$-module of finite type.

A.7. For any map of rings $\rho: R \rightarrow A$, let $\mathcal{S}_{a, R}$ denote the groupoid of pairs $\left(a_{R}, \alpha\right)$, where $a_{R} \in \mathcal{S}_{R}$ and $\alpha: \rho^{*}\left(a_{R}\right) \simeq a$ is an isomorphism in $\mathcal{S}_{A}$. Since $\mathcal{S}$ is an algebraic stack, for any deformation situation as in ([2], 2.2) and $a \in \mathcal{S}_{A}$ the functor

$$
\mathcal{S}_{a, A^{\prime} \times{ }_{A} B} \rightarrow \mathcal{S}_{a, A^{\prime}} \times \mathcal{S}_{a, B}
$$

is an equivalence of categories ([2], converse of 5.3 on p. 182). It follows that for any $\mathcal{S}$ deformation situation as above, the product $\mathcal{A}^{\prime} \times{ }_{\mathcal{A}} \mathcal{B}$ exists in the category of $\mathcal{S}$-rings.

The following conditions $\left(\mathrm{S}^{\mathcal{S}}\right)$ and $\left(\mathrm{S}^{\mathcal{S}}\right)$ will be referred to as Schlessinger's conditions.

Condition $\left(\mathrm{S}^{\mathcal{S}}\right)$. Given an $\mathcal{S}$-deformation situation as in (A.6) and $a \in F_{\mathcal{A}}$, the natural functor

$$
F_{a, \mathcal{A}^{\prime} \times \mathcal{A} \mathcal{B}} \longrightarrow F_{a, \mathcal{A}^{\prime}} \times F_{a, \mathcal{B}}
$$

is an equivalence of categories.

A.8. If $\mathcal{A}$ is an $\mathcal{S}$-ring, and if $I$ is an $A_{0}$-module of finite type, let $A[I]$ be the ring whose underlying group is $A \oplus I$ and whose multiplication law is given by

$$
(a, i) \cdot\left(a^{\prime}, i^{\prime}\right):=\left(a a^{\prime}, a i^{\prime}+a^{\prime} i\right) .
$$

There is a natural retraction

$$
\operatorname{Spec}(A[I]) \longrightarrow \operatorname{Spec}(A), \quad A \longrightarrow A[I], \quad a \longmapsto(a, 0)
$$

which gives $A[I]$ the structure of an $\mathcal{S}$-ring which we denote by $\mathcal{A}[I]$. It follows from the definition of $\mathcal{A}[I]$ that

$$
\mathcal{A}[I] \times{ }_{\mathcal{A}} \mathcal{A}[I] \simeq \mathcal{A}[I \oplus I],
$$

and so just as in the case when $\mathcal{S}$ is a scheme ([43], 2.10), $\mathcal{A}[I]$ has the structure of an $A_{0}$-module object in the category of $\mathcal{S}$-rings. It follows from this that if $\left(\mathrm{S}^{\mathcal{S}}\right)$ holds, then 
given $a_{0} \in F_{\mathcal{A}_{0}}$ the isomorphism classes of objects in $F_{a_{0}, \mathcal{A}_{0}[I]}$ has a natural structure of an $A_{0}$-module. We denote this module by $\mathrm{T}_{F / \mathcal{S}, a_{0}}(I)$. Similarly, if $\operatorname{Aut}_{a_{0}}\left(\mathcal{A}_{0}[I]\right)$ denotes the set of automorphisms of $\left.a_{0}\right|_{\mathcal{A}_{0}[I]}$ which induce the identity on $a_{0}$ then $\operatorname{Aut}_{a_{0}}\left(\mathcal{A}_{0}[I]\right)$ also has a natural structure of an $A_{0}$-module.

Condition $\left(\mathrm{S}^{\mathcal{S}}\right)$. The $A_{0}$-modules $\mathrm{T}_{F / \mathcal{S}, a_{0}}(I)$ and $\operatorname{Aut}_{a_{0}}\left(\mathcal{A}_{0}[I]\right)$ are of finite type.

Remark A.9. Applying the above discussion to $F \rightarrow S$ one obtains conditions $\left(\mathrm{S}^{S}\right)$ and $\left(\mathrm{S} 2^{S}\right)$. These conditions are denoted $\left(\mathrm{S}^{\prime}\right)$ and $(\mathrm{S} 2)$ in ([2], 2.3 and 2.5), and we follow the notation of (loc. cit.) in this situation. If ( $\left.\mathrm{S}^{\prime}\right)$ holds, then we obtain modules

$$
\mathrm{T}_{F / S, a_{0}}(I) \text { and } \operatorname{Aut}_{a_{0}}\left(A_{0}[I]\right)
$$

which are denoted $D_{a_{0}}(I)$ and $\operatorname{Aut}_{a_{0}}\left(A_{0}+I\right)$ in (loc. cit., 2.4 and 5.3). In the rest of the paper, we use the notation (A.9.1) for these modules.

We will also need a generalization of the notion of an obstruction theory ([2], 2.6):

Definition A.10. An obstruction theory for $F / \mathcal{S}$ consists of the following data:

(i) For every morphism of $\mathcal{S}$-rings $\mathcal{A} \rightarrow \mathcal{A}_{0}$ as in (A.6) and $a \in F_{\mathcal{A}}$, a functor

$$
\mathcal{O}_{a}:\left(A_{0} \text {-modules of finite type }\right) \longrightarrow\left(A_{0} \text {-modules of finite type }\right) \text {. }
$$

(ii) For every diagram $\mathcal{A}^{\prime} \rightarrow \mathcal{A} \rightarrow \mathcal{A}_{0}$ as in (A.6), an element $o_{a}\left(\mathcal{A}^{\prime}\right) \in \mathcal{O}_{a}(I)$ which is zero if and only if there exists a lifting of $a$ to $\mathcal{A}^{\prime}$.

This data is required to be functorial and linear in $\mathcal{A}_{0}$ and $I$.

A.11. We will consider the following additional conditions on the modules $\mathrm{T}_{F / \mathcal{S}, a_{0}}, \mathcal{O}_{a}(I)$, and $\operatorname{Aut}_{a_{0}}\left(\mathcal{A}_{0}[I]\right)$ :

(i) Compatibility with étale localization: With notation as above, if $\mathcal{A} \rightarrow \mathcal{B}$ is an étale morphism of $\mathcal{S}$-rings (i.e. $A \rightarrow B$ is étale), then for any $a \in F_{\mathcal{A}}$ with image $b \in F_{\mathcal{B}}$

$$
\begin{aligned}
\mathrm{T}_{F / \mathcal{S}, b_{0}}\left(I \otimes B_{0}\right) & \simeq \mathrm{T}_{F / \mathcal{S}, a_{0}}(I) \otimes B_{0}, \\
\operatorname{Aut}_{b_{0}}\left(\mathcal{B}_{0}\left[I \otimes B_{0}\right]\right) & \simeq \operatorname{Aut}_{a_{0}}\left(\mathcal{A}_{0}[I]\right) \otimes B_{0},
\end{aligned}
$$

and

$$
\mathcal{O}_{b}\left(I \otimes B_{0}\right) \simeq \mathcal{O}_{a}(I) \otimes B_{0}
$$

where $B_{0}$ (resp. $\left.b_{0}\right)$ denotes $B \otimes_{A} A_{0}$ (resp. the pullback of $b$ to $B_{0}$ ).

(ii) Effectivity of formal objects: If $\mathfrak{m} \subset A_{0}$ is a maximal ideal and $\hat{A}$ the completion of $A_{0}$ along $\mathfrak{m}$, then

$$
\begin{aligned}
\mathrm{T}_{F / \mathcal{S}, a_{0}}(I) \otimes \hat{A} & \simeq \lim _{\longleftarrow} \mathrm{T}_{F / \mathcal{S}, a_{0}}\left(I / \mathfrak{m}^{n} I\right), \\
\operatorname{Aut}_{a_{0}}\left(\mathcal{A}_{0}[I]\right) \otimes \hat{A} & \simeq \lim _{\longleftarrow} \operatorname{Aut}_{a_{0}}\left(\mathcal{A}_{0}\left[I / \mathfrak{m}^{n} I\right]\right),
\end{aligned}
$$

and

$$
\mathcal{O}_{a_{0}}(I) \otimes \hat{A} \hookrightarrow \lim _{\longleftarrow} \mathcal{O}_{a_{0}}\left(I / \mathfrak{m}^{n} I\right)
$$

(iii) Constructibility: There exists an open dense set of points $p$ in $\operatorname{Spec}\left(A_{0}\right)$ with $\kappa(p)$ of finite type over $S$ such that

$$
\mathrm{T}_{F / \mathcal{S}, a_{0}}(I) \otimes \kappa(p) \simeq \mathrm{T}_{F / \mathcal{S}, a_{0}}(I \otimes \kappa(p)),
$$




$$
\operatorname{Aut}_{a_{0}}\left(\mathcal{A}_{0}[I]\right) \otimes \kappa(p) \simeq \operatorname{Aut}_{a_{0}}\left(\mathcal{A}_{0}[I \otimes \kappa(p)]\right),
$$

and

$$
\mathcal{O}_{a}(I) \otimes \kappa(p) \subset \mathcal{O}_{a}(I \otimes \kappa(p)) .
$$

Theorem A.12. Let $m: F \rightarrow \mathcal{S}$ be a limit preserving $\mathcal{S}$-stack(A.5), and suppose an obstruction theory $\mathcal{O}$ is given. Then $F$ is an algebraic stack if the following conditions hold:

(i) The conditions $\left(\mathrm{S}^{\mathcal{S}}\right),\left(\mathrm{S}^{\mathcal{S}}\right)$, and the conditions on $\mathrm{T}_{F / \mathcal{S}, a_{0}}(I)$ and $\operatorname{Aut}_{a_{0}}\left(\mathcal{A}_{0}[I]\right)$ in (A.11) hold.

(ii) If $\hat{\mathcal{A}}$ is a complete local $\mathcal{S}$-ring with residue field of finite type over $S$, then the natural functor

$$
F_{\hat{\mathcal{A}}} \longrightarrow \lim _{\longleftarrow} F_{\hat{\mathcal{A}} / \mathfrak{m}^{n}}
$$

is faithful and has dense image.

(iii) The obstruction theory $\mathcal{O}$ satisfies the conditions in (A.11). In addition, for every reduced noetherian ring $A_{0}, a_{0} \in F_{A_{0}}$ and $I$ an $A_{0}$-module of finite type, the map

$$
\overline{\mathcal{S}}_{m\left(a_{0}\right), A_{0}[I]} \longrightarrow \mathcal{O}_{a_{0}}(I)
$$

which sends a lifting $b$ of $m\left(a_{0}\right)$ to $A_{0}[I]$ to the obstruction

$$
o_{a_{0}}\left(\left(A_{0}[I], b\right)\right) \in \mathcal{O}_{a_{0}}(I)
$$

is an $A_{0}$-module homomorphism. Here $\left(A_{0}[I], b\right)$ denotes the $\mathcal{S}$-ring induced by $b$ which comes with a natural morphism of $\mathcal{S}$-rings

$$
\left(A_{0}[I], b\right) \longrightarrow \mathcal{A}_{0}
$$

where $\mathcal{A}_{0}$ denotes the $\mathcal{S}$-ring obtained from $A_{0}$ via $m\left(a_{0}\right)$. The $A_{0}$-module structure on $\overline{\mathcal{S}}_{m\left(a_{0}\right), A_{0}[I]}$ is that described in (A.8).

(iv) If $\mathcal{A}_{0}$ is of finite type over $S, a_{0} \in F_{\mathcal{A}_{0}}$ an object, and $\phi$ an automorphism of $a_{0}$ which induces the identity in $F_{\kappa}$ ( $\kappa$ viewed as a $\mathcal{S}$-ring) for a dense set of points $A_{0} \rightarrow \kappa$ with $\kappa$ of finite type over $S$, then $\phi$ is the identity over some dense open subset of $\operatorname{Spec}\left(A_{0}\right)$.

Proof. We show that $F$ is relatively representable by verifying the conditions of the following:

Proposition A.13. A limit preserving stack $F / S$ is relatively representable if the following conditions hold:

(i) Schlessinger's conditions ( $\left.\mathrm{S}^{\prime}\right)$ and (S2) hold, and in addition the modules $\mathrm{Aut}_{a_{0}}\left(A_{0}[I]\right)$ are $A_{0}$-modules of finite type.

(ii) If $\hat{A}$ is a complete local ring with residue field of finite type over $S$, then the natural functor

$$
F(\hat{A}) \longrightarrow \lim _{\longleftarrow} F\left(\hat{A} / \mathfrak{m}^{n}\right)
$$

is faithful. In addition, given two objects $x, y \in F(\hat{A})$ and a compatible family of isomorphisms $\xi_{n}: x_{n} \rightarrow y_{n}$ between the reductions modulo $\mathfrak{m}^{n}$, there exists for every $n$ an isomorphism $\xi: x \rightarrow y$ inducing $\xi_{n}$.

(iii) The modules $\mathrm{T}_{F / S, a_{0}}(I)$ and $\operatorname{Aut}_{a_{0}}\left(A_{0}[I]\right)$ satisfy the conditions for $D$ in $([2], 4.1)$. 
(iv) If $a_{0} \in F\left(A_{0}\right)$ is an element over a ring of finite type over $S$, and if $\phi$ is an automorphism of $a_{0}$ which induces the identity in $F(\kappa)$ for a dense set of points $A_{0} \rightarrow \kappa$, then $\phi$ is the identity over some dense open subset of $\operatorname{Spec}\left(A_{0}\right)$.

Proof. This follows from ([2], 5.3) applied to the Isom-functors.

A.14 (Verification of $\left(\mathrm{S}^{\prime}\right)$ ). Given a deformation situation as in $([2], 2.2)$ and $a \in F_{A}$, we have to show that the natural functor

$$
F_{a, A^{\prime} \times{ }_{A} B} \longrightarrow F_{a, A^{\prime}} \times F_{a, B}
$$

is an equivalence of categories.

To see that (A.14.1) is essentially surjective, suppose given $a^{\prime} \in F_{a, A^{\prime}}$ and $b \in F_{a, B}$. The map

$$
m \circ a: \operatorname{Spec}(A) \longrightarrow \mathcal{S}
$$

gives $A$ the structure of an $\mathcal{S}$-ring $\mathcal{A}$, and similarly $a^{\prime}$ and $b$ define $\mathcal{S}$-rings $\mathcal{A}^{\prime}$ and $\mathcal{B}$. Moreover, the isomorphisms $\left.a^{\prime}\right|_{A} \simeq a$ and $\left.b\right|_{A} \simeq a$ define morphisms of $\mathcal{S}$-rings

$$
\mathcal{A}^{\prime} \longrightarrow \mathcal{A}, \mathcal{B} \longrightarrow \mathcal{A}
$$

and hence we have a $\mathcal{S}$-deformation situation as in (A.6) and an object in $F_{a, \mathcal{A}^{\prime}} \times F_{a, \mathcal{B}}$. By $\left(\mathrm{S}^{\mathcal{S}}\right)$, there exists an object in $F_{a, \mathcal{A}^{\prime} \times \mathcal{A} \mathcal{B}}$ inducing this object, and hence (A.14.1) is essentially surjective.

For the full faithfulness, suppose $a_{1}, a_{2} \in F_{a, A^{\prime} \times{ }_{A} B}$. Given an isomorphism $\left(f^{\prime}, f_{B}\right)$ in $F_{a, A^{\prime}} \times F_{a, B}$, we get an isomorphism $\left(m\left(f^{\prime}\right), m\left(f_{B}\right)\right)$ in $\mathcal{S}_{m(a), A^{\prime}} \times \mathcal{S}_{m(a), B}$, and hence by $\left(\mathrm{S}^{\prime}\right)$ for $\mathcal{S}$ an isomorphism $f: m\left(a_{1}\right) \simeq m\left(a_{2}\right)$ in $\mathcal{S}_{a, A^{\prime} \times{ }_{A} B}$. The element $a_{1}$ gives in the same manner as in the preceding paragraph $A^{\prime}, A$, and $B$ the structure of $\mathcal{S}$-rings $\mathcal{A}^{\prime}, \mathcal{A}$, and $\mathcal{B}$ and the morphism $f$ allows us to view $a_{2}$ as an object in $F_{a, \mathcal{A}^{\prime} \times \mathcal{A}_{\mathcal{B}}}$ and the morphism $\left(f^{\prime}, f_{B}\right)$ as a morphism in $F_{a, \mathcal{A}^{\prime}} \times F_{a, \mathcal{B}}$. From $\left(\mathrm{S}^{\mathcal{S}}\right)$ it therefore follows that (A.14.1) is full. Similarly, given two isomorphisms $f_{1}, f_{2}: a_{1} \rightrightarrows a_{2}$ in $F_{a, A^{\prime} \times{ }_{A} B}$ which define the same morphism in $F_{a, A^{\prime}} \times F_{a, B}$, we must by $\left(\mathrm{S}^{\prime}\right)$ for $\mathcal{S}$ have $m\left(f_{1}\right)=m\left(f_{2}\right)$. Therefore we can view $f_{1}$ and $f_{2}$ as morphisms in $F_{a, \mathcal{A}^{\prime} \times \mathcal{A}} \mathcal{B}$, where we give $A^{\prime}, A$, and $B$ the structure of $\mathcal{S}$-rings via $a_{1}$. Therefore, $\left(\mathrm{S}^{\mathcal{S}}\right)$ also implies that (A.14.1) is faithful. This proves that (S1') holds.

A.15 (Verification of (S2), finiteness of $\operatorname{Aut}_{a_{0}}(I)$, and (A.13 (iii))). Let $A_{0}$ be a reduced noetherian $S$-ring, and let $a_{0} \in F_{A_{0}}$. The composite $m \circ a_{0}$ gives $A_{0}$ the structure of a $\mathcal{S}$-ring which we denote by $\mathcal{A}_{0}$. We claim that there is a natural 8-term exact sequence of $A_{0}$-modules

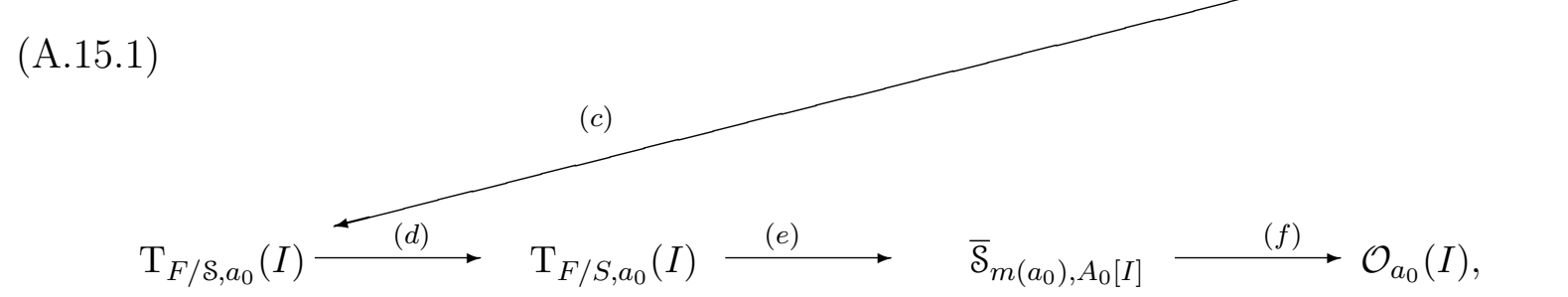

where $\operatorname{Aut}_{m\left(a_{0}\right)}\left(A_{0}[I]\right)$ is the module of infinitesimal automorphisms of $m\left(a_{0}\right) \in \mathcal{S}$. The maps $(a),(b),(d)$, and $(f)$ are the maps induced by functoriality. The map $(c)$ is defined as 
follows. Let $h: \operatorname{Spec}\left(A_{0}[I]\right) \rightarrow \mathcal{S}, f: \operatorname{Spec}\left(A_{0}[I]\right) \rightarrow F, \eta: h \simeq m \circ f$ be the data defining the element over $A_{0}[I]$ defined by $a_{0}$. The map sends an automorphism $\phi: h \rightarrow h$ to the element in $\mathrm{T}_{F / \mathcal{S}, A_{0}[I]}$ defined by the maps $h$ and $f$ together with the isomorphism $\eta \circ \phi$. It follows from unwinding the construction of the module structures that this in fact is an $A_{0^{-}}$ module homomorphism. The map $(f)$ is described in (A.12 (iii)). The exactness of (A.15.1) is tautological.

Since all the modules in (A.15.1) except $\operatorname{Aut}_{a_{0}}\left(A_{0}[I]\right)$ and $T_{F / S, a_{0}}(I)$ are known to be of finite type, it follows that (S2) holds and that $\operatorname{Aut}_{a_{0}}\left(A_{0}[I]\right)$ is of finite type. Moreover, combining a diagram chase with the fact that the additional conditions ([2], 4.1) hold for $\operatorname{Aut}_{m\left(a_{0}\right)}\left(A_{0}[I]\right)$ and $\overline{\mathcal{S}}_{m\left(a_{0}\right)}\left(A_{0}[I]\right)([2]$, converse to 5.3 on p. 182), one sees that (A.13 (iii)) holds.

A.16 (Verification of (A.13 (ii))). The faithfulness of (A.13.1) can be shown as follows. If $a_{1}, a_{2} \in F_{\hat{A}}$ and $f_{1}, f_{2}: a_{1} \rightrightarrows a_{2}$ are morphisms which define the same morphism in $\lim _{\longleftarrow} F_{\hat{A} / \mathfrak{m}^{n}}$, then $m\left(f_{1}\right)$ and $m\left(f_{2}\right)$ must be equal since (A.13 (ii)) holds for $\mathcal{S}$ by ([2], converse to 5.3 on p. 182). View $\hat{A}$ as an $\mathcal{S}$-ring via $m\left(f_{1}\right)$. Then $f_{1}$ and $f_{2}$ are morphisms in $F_{\hat{\mathcal{A}}}$ which induce the same map in $\lim _{\leftarrow} F_{\hat{\mathcal{A}} / \mathfrak{m}^{n}}$ and hence must be equal by (A.12 (ii)).

Next suppose $a_{1}, a_{2} \in F_{\hat{A}}$ and that a compatible family of of isomorphisms $\xi_{n}: a_{1, n} \simeq a_{2, n}$ are given. Since $\mathcal{S}$ is an algebraic stack, $m\left(a_{1}\right) \times_{\mathcal{S}} m\left(a_{2}\right)$ is representable by an algebraic space, and hence there exists a unique isomorphism $m(\xi): m\left(a_{1}\right) \simeq m\left(a_{2}\right)$ in $\mathcal{S}_{\hat{A}}$ inducing the isomorphisms $m\left(\xi_{n}\right)$. It follows that we can view $a_{1}$ and $a_{2}$ as objects in $F_{\hat{\mathcal{A}}}$, where $\hat{\mathcal{A}}$ is the $\mathcal{S}$-ring obtained via $m\left(a_{1}\right)$. Then by (A.12 (ii)), there exists for all $n$ an isomorphism $\xi: a_{1} \simeq a_{2}$ inducing $\xi_{n}$.

A.17 (Verification of (A.13 (iv))). If $A_{0}$ is reduced and of finite type over $S, a_{0} \in F_{A_{0}}, \phi$ an automorphism of $a_{0}$ which induces the identity for a dense set of points, then there exists a dense open set of $A_{0}$ such that $m(\phi)$ is the identity automorphism of $m\left(a_{0}\right)$ over this open set by ([2], converse to 5.3). Replacing $A_{0}$ by the ring of a basic open set inside this open set, we can assume that $m(\phi)$ is the identity map. Thus viewing $A_{0}$ as an $\mathcal{S}$-ring via $m\left(a_{0}\right)$ we are reduced to (A.12 (iv)). This completes the proof that $F$ is relatively representable.

A.18. To prove that $F$ admits a smooth cover, it suffices to show that for any smooth cover $b: S^{\prime} \rightarrow \mathcal{S}$ the stack $F^{\prime}:=F \times_{\mathcal{S}} S^{\prime}$ is algebraic. For if $T \rightarrow F^{\prime}$ is a smooth surjective cover, then for any 1-morphism $X \rightarrow F$ from an algebraic space all the arrows in

$$
T \times_{F^{\prime}}\left(F^{\prime} \times_{F} X\right) \longrightarrow F^{\prime} \times_{F} X \simeq S^{\prime} \times_{\mathcal{S}} X \longrightarrow X
$$

are smooth surjective morphisms of algebraic spaces. Hence $T^{\prime}$ also gives a smooth surjective cover of $F$.

If $s: \operatorname{Spec}(A) \rightarrow S^{\prime}$ is a morphism, then giving an object in $F_{A}^{\prime}$ is equivalent to giving a pair $(f, h)$, where $f: \operatorname{Spec}(A) \rightarrow F$ is a 1-morphism and $h: s^{*}(b) \simeq m(f)$ is an isomorphism in $\mathcal{S}$. A morphism $\left(f^{\prime}, h^{\prime}\right) \rightarrow(f, h)$ in $F_{A}^{\prime}$ is the same as a morphism $\eta: f^{\prime} \rightarrow f$ in $F_{A}$ such that $m(\eta) \circ h^{\prime}=h$. In other words, if we view $A$ as a $\mathcal{S}$-ring $\mathcal{A}$ via the map $b$, the category $F_{A}^{\prime}$ is naturally equivalent to the category $F_{\mathcal{A}}$.

This implies that all the hypothesis of ([2], theorem 5.3) hold, except possibly for the existence of an obstruction theory. To construct an obstruction theory $\mathcal{O}^{\prime}$ for $F^{\prime}$ proceed as follows. An infinitesimal extension $A \rightarrow A_{0}$ over $S^{\prime}$ can be viewed via $b$ as an infinitesimal 
extension of $\mathcal{S}$-rings $\mathcal{A} \rightarrow \mathcal{A}_{0}$. Moreover, an element $a \in F_{A}^{\prime}$ defines in a natural way an element in $F_{\mathcal{A}}$ which we also denote by $a$. Define the functor $\mathcal{O}_{a}^{\prime}$ to be the functor $\mathcal{O}_{a}$ of (A.10) corresponding to this data. Given a deformation situation $A^{\prime} \rightarrow A \rightarrow A_{0}$ over $S^{\prime}$, we can view it as a $\mathcal{S}$-deformation situation, and hence get an element $o_{a}(I) \in \mathcal{O}_{a}^{\prime}(I)$. It is immediate that $\left(\mathcal{O}_{a}^{\prime}, o_{a}\right)$ satisfies the axioms of an obstruction theory ([2], 2.6) and ([2], 4.1). This proves that $F^{\prime}$ is an algebraic stack, and hence $F$ is also algebraic.

Remark A.19. From the proof it follows that it suffices to construct local obstruction theories $\mathcal{O}_{a}$ in the sense of $([2], 4.9)$ translated into the language of $\mathcal{S}$-schemes.

\section{Appendix B. Restatement using log geometry}

In this section we restate (A.12) in the case when $\mathcal{S}=\mathcal{L} o g_{S}$, thereby obtaining a more usable form of (A.12) for the application to log geometry. Throughout this section we work over a fixed fine log scheme $S$ whose underlying scheme $\stackrel{\circ}{S}$ is of finite type over an excellent Dedekind ring.

Definition B.1. A log stack over $S$ is a pair $F=\left(\stackrel{\circ}{F}, \mathcal{M}_{F}\right)$, where $\stackrel{\circ}{F}$ is a stack over the category of $\stackrel{\circ}{S}$-schemes and $\mathcal{M}_{F}: \stackrel{\circ}{F} \rightarrow \mathcal{L}_{o} g_{S}$ is a morphism of stacks.

Remark B.2. In the case when $\stackrel{\circ}{F}$ is an algebraic stack, it follows from ([39], 5.8) that giving the morphism $\mathcal{M}_{F}$ is equivalent to giving a fine $\log$ structure $\mathcal{M}$ on $\stackrel{\circ}{F}$ in the sense of (loc. cit., 5.1) together with a morphism of log algebraic stacks $(\stackrel{\circ}{F}, \mathcal{M}) \rightarrow S$.

B.3. If $F$ is a $\log$ stack and $t: T \rightarrow \stackrel{\circ}{F}$ is a 1 -morphism with $T$ a scheme, then $\mathcal{M}_{F} \circ t: T \rightarrow$ $\mathcal{L}_{\log }$ induces a $\log$ structure denoted $t^{*} \mathcal{M}_{F}$ on $T$ together with a morphism of log schemes $\left(T, t^{*} \mathcal{M}_{F}\right) \rightarrow S$

If $f: X \rightarrow S$ is a $\log$ scheme over $S$, then we define a morphism $X \rightarrow F$ to be a pair $\left(x, x^{b}\right)$, where $x: \stackrel{\circ}{X} \rightarrow \stackrel{\circ}{F}$ is a 1-morphism and $x^{b}: x^{*} \mathcal{M}_{F} \rightarrow \mathcal{M}_{X}$ is a morphism of log structures on $\stackrel{\circ}{X}$ such that the resulting morphism of log schemes

$$
X \longrightarrow\left(\stackrel{\circ}{X}, x^{*} \mathcal{M}_{F}\right)
$$

is a morphism over $S$. If $\left(x_{i}, x_{i}^{b}\right)(i=1,2)$ are two morphisms $X \rightarrow F$, then a morphism $\left(x_{1}, x_{1}^{b}\right) \rightarrow\left(x_{2}, x_{2}^{b}\right)$ is an isomorphism $\epsilon: x_{1} \rightarrow x_{2}$ in $\stackrel{\circ}{F_{X}}$ such that the resulting diagram

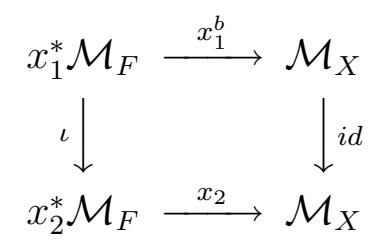

commutes, where $\iota$ denotes the isomorphism induced by $\epsilon$. We denote by $F_{X}$ the groupoid of maps $\left(x, x^{b}\right): X \rightarrow F$ for which the map $x^{b}: x^{*} \mathcal{M}_{F} \rightarrow F$ is an isomorphism. 
Notational Remark B.4. One has to be careful in distinguishing between $F_{X}$ and the groupoid of all morphisms $X \rightarrow F$ (not necessarily strict). We suggest the notation $F(X)$ for the latter category since this is the fiber over $X$ of the fibered category over the category of $\log$ schemes defined by $F$. The notation $F_{X}$ for the groupoid of strict morphisms $X \rightarrow F$ comes from the fact that $F_{X}$ is isomorphic to the fiber product of

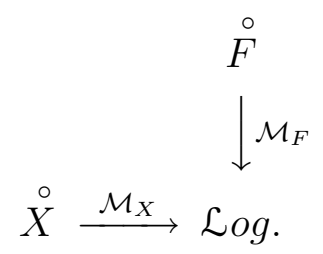

B.5. Define the category of noetherian $\log$ rings $\mathcal{C}$ as follows. $\mathcal{C}^{\text {op }}$ is the subcategory of $\log _{S}$ of $\log$ schemes whose underlying schemes are isomorphic to the spectra of noetherian rings and whose morphisms are strict morphisms of log schemes. We denote the objects of $\mathcal{C}$ by caligraphic letters and the underlying rings by unadorned letters (i.e. an object $\mathcal{A} \in \mathcal{C}$ has underlying scheme $\operatorname{Spec}(A))$. If $\mathcal{A} \in \mathcal{C}$, and if $I$ is an $A$-module of finite type, then we denote by $\mathcal{A}[I]$ the object of $\mathcal{C}$ with underlying ring $A[I]$ and $\log$ structure obtained from $A$ by pullback via the retraction $A \rightarrow A[I]$. There is a canonical morphism $\mathcal{A}[I] \rightarrow \mathcal{A}$ in $\mathcal{C}$. By construction there is a contravariant functor

$$
\text { Spec }: \mathcal{C} \longrightarrow \text { (category of fine log schemes over } S \text { ). }
$$

Definition B.6. A logarithmic deformation situation is a diagram in $\mathcal{C}$

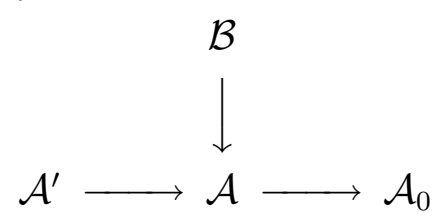

such that the underlying maps of rings satisfy (A.6 (i))-(A.6 (iv)).

Remark B.7. As discussed in (A.7), for any logarithmic deformation situation as above the product $\mathcal{A}^{\prime} \times{ }_{\mathcal{A}} \mathcal{B}$ exists in $\mathcal{C}$.

Rewriting condition $\left(\mathrm{S}^{\mathcal{S}}\right)$ in the case when $\mathcal{S}=\mathcal{L} o g_{S}$ we obtain:

Condition ( $\left.\mathrm{S} 1^{\log }\right)$ For every logarithmic deformation situation and $a \in F_{\mathcal{A}}$, the canonical functor

$$
F_{a, \mathcal{A}^{\prime} \times \mathcal{A}^{\mathcal{B}}} \rightarrow F_{a, \mathcal{A}^{\prime}} \times F_{a, \mathcal{B}}
$$

is an equivalence of categories.

B.8. Suppose a logarithmic deformation situation is given, together with an object $a \in F_{\mathcal{A}}$. If the condition ( $\left.\mathrm{S} 1^{\log }\right)$ holds, then for any $A_{0}$-module of finite type $I$, the set $\mathrm{T}_{F / S, a_{0}}(I):=$ $\bar{F}_{a_{0}, \mathcal{A}_{0}[I]}$ has a natural $A_{0}$-module structure (A.8). Similarly, if $\operatorname{Aut}_{a_{0}}\left(\mathcal{A}_{0}[I]\right)$ denotes the set of automorphisms of $a_{0}$ in $F_{\mathcal{A}_{0}[I]}$ which induce the identity over $\mathcal{A}_{0}$, then $\operatorname{Aut}_{a_{0}}\left(\mathcal{A}_{0}[I]\right)$ has a natural $A_{0}$-module structure.

Example B.9. In the case when $F$ is a fine log scheme, then ([28], 3.9) shows that there is a natural isomorphism

$$
\mathrm{T}_{F / S, a_{0}}(I) \simeq \operatorname{Hom}_{A_{0}}\left(a_{0}^{*} \Omega_{F / S}^{1}, I\right),
$$

where $\Omega_{F / S}^{1}$ denotes the sheaf of logarithmic differentials. 
Condition $\left(\mathrm{S} 2^{\log }\right)$ The modules $\mathrm{T}_{F / S, a_{0}}(I)$ and Aut $_{a_{0}}\left(\mathcal{A}_{0}[I]\right)$ are $A_{0}$-modules of finite type.

Definition B.10. An obstruction theory for $F / S$ consists of data as follows:

(i) For every morphism $\mathcal{A} \rightarrow \mathcal{A}_{0}$ as in (B.6) and $a \in F_{\mathcal{A}}$ a functor

$$
\mathcal{O}_{a}:\left(A_{0} \text {-modules of finite type }\right) \longrightarrow\left(A_{0} \text {-modules of finite type }\right) \text {. }
$$

(ii) For each diagram $\mathcal{A}^{\prime} \rightarrow \mathcal{A} \rightarrow \mathcal{A}_{0}$ as in (B.6), an element $o_{a}\left(\mathcal{A}^{\prime}\right) \in \mathcal{O}_{a}(I)$ which is zero if and only if $\bar{F}_{a, \mathcal{A}^{\prime}}$ is not empty.

The data $\left\{\mathcal{O}_{a}, o_{a}(I)\right\}$ is required to be functorial and linear in $I$.

Let $\mathcal{A} \rightarrow \mathcal{A}_{0}$ be a morphism in $\mathcal{C}$ such that $\operatorname{Ker}\left(A \rightarrow A_{0}\right)$ is nilpotent and $A_{0}$ is reduced. Suppose $A^{\prime} \rightarrow A$ is a surjection with kernel an $A_{0}$-module of finite type $I$ and that $a \in F_{\mathcal{A}}$. Then for each lifting of $\mathcal{M}_{A}$ to $A^{\prime}$, we get a diagram as in (B.6), and hence via $o_{a}$ an element of $\mathcal{O}_{a}(I)$. In other words, $o_{a}$ defines a set map

$$
\text { (liftings of } \left.\mathcal{M}_{A} \text { to } A^{\prime}\right) \longrightarrow \mathcal{O}_{a}(I) \text {. }
$$

Since $\mathcal{L} g_{S}$ is algebraic, if we take $A^{\prime}=A[I]$, then the set of isomorphism classes of liftings of $\mathcal{M}_{A}$ has a natural module structure. If we denote this module by $\mathrm{T}_{\mathcal{L o g}_{S}, \mathcal{A}}(I)$ then the above defines a map

$$
\rho_{I}: T_{\log _{S}, \mathcal{A}}(I) \longrightarrow \mathcal{O}_{a}(I)
$$

We make the following addition requirement of the obstruction theory:

(iii) $\rho_{I}$ is an $A_{0}$-module homomorphism.

B.11. Let $a \in F_{\mathcal{A}}$ be an object with $A$ of finite type over $\stackrel{\circ}{S}$, and let the subscript $A_{0}, B_{0}$, etc. denote the objects obtained by tensor product with $A_{\text {red }}$.

(i) Compatibility with strict étale localization: If $\phi: \mathcal{A} \rightarrow \mathcal{B}$ is a morphism in $\mathcal{C}$ whose underlying morphism of rings is étale, and if $b \in F_{B}$ is the image of $a \in F_{A}$, then

$$
\begin{gathered}
\mathrm{T}_{F / S, b_{0}}(I) \simeq \mathrm{T}_{F / S, a_{0}}(I) \otimes B_{0}, \\
\operatorname{Aut}_{b_{0}}\left(\mathcal{B}_{0}\left[I \otimes B_{0}\right]\right) \simeq \operatorname{Aut}_{a_{0}}\left(\mathcal{A}_{0}[I]\right) \otimes B_{0},
\end{gathered}
$$

and

$$
\mathcal{O}_{b}\left(I \otimes B_{0}\right) \simeq \mathcal{O}_{a}(I) \otimes B_{0}
$$

(ii) Effectivity of formal objects: If $\mathfrak{m} \subset A_{0}$ is a maximal ideal and $\hat{A}$ the completion of $A_{0}$ along $\mathfrak{m}$, then

$$
\begin{aligned}
\mathrm{T}_{F / S, a_{0}}(I) \otimes \hat{A} & \simeq \lim _{\longleftarrow} \mathrm{T}_{F / S, a_{0}}\left(I / \mathfrak{m}^{n} I\right), \\
\operatorname{Aut}_{a_{0}}\left(\mathcal{A}_{0}[I]\right) \otimes \hat{A} & \simeq \lim _{\longleftarrow} \operatorname{Aut}_{a_{0}}\left(\mathcal{A}_{0}\left[I / \mathfrak{m}^{n} I\right]\right),
\end{aligned}
$$

and

$$
\mathcal{O}_{a_{0}}(I) \otimes \hat{A} \hookrightarrow \lim _{\longleftarrow} \mathcal{O}_{a_{0}}\left(I / \mathfrak{m}^{n} I\right) .
$$

(iii) Contructibility: There exists an open dense set of points of finite type $p$ in $\operatorname{Spec}\left(A_{0}\right)$ such that

$$
\begin{aligned}
\mathrm{T}_{F / S, a_{0}}(I) \otimes \kappa(p) & \simeq \mathrm{T}_{F / S, a_{0}}(I \otimes \kappa(p)) \\
\operatorname{Aut}_{a_{0}}\left(\mathcal{A}_{0}[I]\right) \otimes \kappa(p) & \simeq \operatorname{Aut}_{a_{0}}\left(\mathcal{A}_{0}[I \otimes \kappa(p)]\right),
\end{aligned}
$$


and

$$
\mathcal{O}_{a}(I) \otimes \kappa(p) \subset \mathcal{O}_{a}(I \otimes \kappa(p))
$$

Rewriting (A.12), we obtain the following:

Theorem B.12. Let $F / S$ be a log stack with $\stackrel{\circ}{F}$ limit preserving. Suppose that an obstruction theory $\mathcal{O}$ for $F$ as in (B.10) is given which satisfies the conditions in (B.11). Then $\stackrel{\circ}{F}$ is an algebraic stack if the following conditions hold:

(i) The conditions $\left(\mathrm{S} 1^{\log }\right)$, and ( $\left.\mathrm{S} 2^{\log }\right)$ hold, and the associated modules satisfy the conditions of (B.11).

(ii) For every log ring $\hat{\mathcal{A}}$ such that $\hat{A}$ is complete and local with residue field of finite type over $\stackrel{\circ}{S}$, the natural functor

$$
F_{\hat{\mathcal{A}}} \longrightarrow \lim F_{\hat{\mathcal{A}} / \mathfrak{m}^{n}}
$$

is faithful and has dense image.

(iii) Suppose given a reduced object $\mathcal{A}_{0}$ of $\mathcal{C}$ of finite type over $\stackrel{\circ}{S}$, an object $a_{0} \in F_{\mathcal{A}_{0}}$, and an automorphism $\phi$ of $a_{0}$. If $\phi$ induces the identity in $F_{\kappa}$ ( $\kappa$ viewed as a log ring with the $\log$ structure induced by $\mathcal{M}_{\mathcal{A}_{0}}$ ) for a dense set of points $A_{0} \rightarrow \kappa$ with $\kappa$ of finite type over $\stackrel{\circ}{S}$, then $\phi$ is the identity over some dense open subset of $\operatorname{Spec}\left(A_{0}\right)$.

Remark B.13. As in (A.19) it suffices to construct local obstruction theories $\mathcal{O}_{a}$. For example, one can always assume that a chart is given for the $\log$ structure on $\mathcal{A}_{0}$, and the obstruction theory may depend on a choice of chart.

\section{REFERENCES}

[1] M. Artin, Algebraic construction of Brieskorn's resolutions, J. Alg. 29 (1974), 330-348.

[2] _ Versal deformations and algebraic stacks, Invent. Math. 27 (1974), 165-189.

[3] _ Algebraization of formal moduli II. Existence of modifications, Ann. Math. (2) 91 (1970), 88-135.

[4] _ Algebraic approximation of structures over complete local rings, Inst. Hautes Études Sci. Publ. Math. 36 (1969), 23-58.

[5] _ Algebraization of formal moduli. I, Global Analysis (Papers in Honor of K. Kodaira), Univ. Tokyo Press, Tokyo (1969), 21-71.

[6] _ On isolated rational singularities of surfaces, Amer. J. Math. 88 (1966), 129-136.

[7] M. Artin, A. Grothendieck, and J.-L. Verdier, Théorie des topos et cohomologie étale des schémas, Lecture Notes in Mathematics 269, 270, 305, Springer-Verlag, Berlin (1971).

[8] A. Ash, D. Mumford, M. Rapoport, and Y. Tai, Smooth compactification of locally symmetric varieties, Math. Sci. Press., Brookline, Mass. (1975).

[9] W. L. Baily, Jr. and A. Borel, Compactification of arithmetic quotients of bounded symmetric domains. Ann. of Math. (2) 84 (1966), 442-528.

[10] A. Borel, Some metric properties of arithmetic quotients of symmetric spaces and an extension theorem, J. Diff. Geom. 6 (1972), 543-560.

[11] P. Deligne, Équations différentielles à points singuliers réguliers, Lecture Notes in Math 163, SpringerVerlag, Berlin (1970).

[12] P. Deligne, J. Milne, A. Ogus, and K.-Y. Shih, Hodge cycles, motives, and Shimura varieties, Lecture Notes in Math 900, Springer-Verlag, Berlin (1982).

[13] P. Deligne and M. Rapoport, Les schémas de modules de courbes elliptiques, Lecture notes in Math 349 , Springer-Verlag, Berlin (1973), 143-316. 
[14] J. Dieudonné and A. Grothendieck, Éléments de géométrie algébrique, Inst. Hautes Études Sci. Publ. Math. 4, 8, 11, 17, 20, 24, 28, 32 (1961-1967).

[15] R. Elkik, Solutions d'équations à coefficients dans un anneau hensélien, Ann. Sci. École Norm. Sup. (4) 6 (1973), 553-603.

[16] R. Friedman, A new proof of the global Torelli theorem for K3 surfaces, Ann. of Math. (2) 120 (1984), $237-269$.

[17] _ Global smoothings of varieties with normal crossings, Ann. of Math. (2) 118 (1983), 75-114.

[18] _ Base change, automorphisms, and stable reduction for type III K3 surfaces, The birational geometry of degenerations, Progr. Math. 29, Birkhauser (1982), 277-298.

[19] R. Friedman and D. Morrison, The birational geometry of degenerations: an overview, The birational geometry of degenerations, Progr. Math. 29, Birkhauser (1982), 1-32.

[20] R. Friedman and F. Scattone, Type III degenerations of K3 surfaces, Invent. Math. 83 (1985), 1-39.

[21] J. Giraud, Cohomologie non abélienne, Die Grundlehren der mathematischen Wissenschaften 179, Springer-Verlag, Berlin (1971).

[22] H. Grauert and R. Remmert, Coherent analytic sheaves, Die Grundlehren der mathematischen Wissenschaften 265, Springer-Verlag, Berlin (1984).

[23] A. Grothendieck, Revêtement Étale et Groupe Fondamental, Lecture Notes in Mathematics 224, Springer-Verlag, Berlin (1971).

[24] R. Hartshorne, Algebraic geometry, Graduate Texts in Mathematics 52, Springer-Verlag, New York (1977).

[25] L. Illusie, Logarithmic spaces (according to K. Kato), Barsotti Symposium in Algebraic Geometry (Abano Terme, 1991), Academic Press, San Diego (1994), 183-203.

[26] T. Kajiwara, Logarithmic compactifications of the generalized Jacobian variety, J. Fac. Sci. Univ. Tokyo Sect. IA, Math. 40 (1993),473-502.

[27] F. Kato, Log smooth deformation theory, Tôhoku Math. J. (2) 48 (1996), 317-354.

[28] K. Kato, Logarithmic structures of Fontaine-Illusie, Algebraic analysis, geometry, and number theory (Baltimore, MD, 1988), Johns Hopkins Univ. Press, Baltimore, MD (1989), 191-224.

[29] Y. Kawamata and Y. Namikawa, Logarithmic deformations of normal crossing varieties and smoothing of degenerate Calabi-Yau varieties, Inv. Math. 118 (1994), 395-409.

[30] D. Knutson, Algebraic spaces, Lecture Notes in Mathematics 203, Springer-Verlag, Berlin (1971).

[31] V. Kulikov, Degenerations of K3 surfaces and Enriques surfaces, Math. USSR Izvestiya 11 (1977), 957989.

[32] G. Laumon and L. Moret-Bailly, Champs algébriques, Ergebnisse der Mathematik 39, Springer-Verlag, Berlin (2000).

[33] J. S. Milne, Shimura varieties and motives, In: Motives (Eds. U. Jannsen, S. Kleiman, J.-P. Serre), Proc. Symp. Pure Math. 55 (1994), 447-523.

[34] D. Mumford, The topology of normal singularities of an algebraic surface and a criterion for simplicity, Inst. Hautes Études Sci. Publ. Math. 9 (1961) 5-22.

[35] Y. Nakajima, Liftings of simple normal crossings log K3 and log Enriques surfaces in mixed characteristic, J. Alg. Geom. 9 (2000) 355-393.

[36] C. Nakayama, Nearby cycles for log smooth families, Compositio Math. 112 (1998), 45-75.

[37] _ Logarithmic étale cohomology, Math. Ann. 308 (1997), 365-404.

[38] A. Ogus, Supersingular K3 crystals, Journées de Géométrie Algébrique de Rennes (Rennes, 1978), Vol. II, Soc. Math. France, Paris (1979).

[39] M. Olsson, Logarithmic geometry and algebraic stacks, to appear in Ann. Sci. École Norm. Sup.

[40] _ Universal log structures on semi-stable varieties, Tohoku Math. J. 55 (2003), 397-438.

[41] _ Log algebraic stacks and moduli of log schemes, Ph.d. thesis, UC Berkeley, May 2001.

[42] F. Scattone, On the compactification of moduli spaces for algebraic K3 surfaces, Mem. Amer. Math. Soc. 374 (1987).

[43] M. Schlessinger, Functors of Artin rings, Trans. Amer. Math. Soc. 130 (1968), 208-222.

[44] N.I. Shepherd-Barron, Extending polarizations on families of K3 surfaces, The birational geometry of degenerations, Progr. Math. 29, Birkhauser (1982), 135-171.

[45] I. Vidal, Contributions à la cohomologie étale des schémas et des log schémas, Thèse, U. Paris-Sud, 2001. 
[46] A. Vistoli, The deformation theory of local complete intersections, preprint, arXiv:alg-geom/9703008 v2. 